Portland State University

PDXScholar

Fall 1-4-2013

\title{
Can Cross-Race Mentoring Help Minority Students and Break Down Prejudice? Mentoring Experiences in Higher Education
}

Jennifer Brooke Rainer

Portland State University

Follow this and additional works at: https://pdxscholar.library.pdx.edu/open_access_etds

Part of the Bilingual, Multilingual, and Multicultural Education Commons, and the Race and Ethnicity Commons

Let us know how access to this document benefits you.

\section{Recommended Citation}

Rainer, Jennifer Brooke, "Can Cross-Race Mentoring Help Minority Students and Break Down Prejudice? Mentoring Experiences in Higher Education" (2013). Dissertations and Theses. Paper 912.

https://doi.org/10.15760/etd.912

This Thesis is brought to you for free and open access. It has been accepted for inclusion in Dissertations and Theses by an authorized administrator of PDXScholar. Please contact us if we can make this document more accessible: pdxscholar@pdx.edu. 
Can Cross-Race Mentoring Help Minority Students and Break Down Prejudice?

Mentoring Experiences in Higher Education

\title{
by
}

Jennifer Brooke Rainer

A thesis submitted in partial fulfillment of the requirements for the degree of

\author{
Master of Science \\ in \\ Sociology \\ Thesis Committee: \\ José Padín, Chair \\ Martha Balshem \\ Yves Labissiere \\ Melissa Thompson
}

Portland State University

2012 


\begin{abstract}
Cross-race mentoring relationships are of interest to the theory and practice of mentoring and they also speak to a longstanding problem in the sociological study of prejudice. The mentoring literature reveals some disagreement regarding the advisability of cross-race matching for young protégés. Some researchers stress same-race matching, while others emphasize the problem this creates for minority's facing a dearth of mentors. Sociologists and psychologists, on the other hand, have amassed evidence showing support for a contact hypothesis, which states frequent intergroup contact between equalstatus members can lead to improved perceptions of the Other. However, to date, the contact hypothesis has not been explored in the context of mentoring relationships between white adults and minority youth. This Thesis hopes to fill these gaps in the literature.
\end{abstract}

The purpose of this Thesis is to better understand the perceptions and understandings mentors and protégés attach to their cross-race mentoring relationship. It is primarily concerned with identifying the conditions that lead to successful outcomes of interracial mentoring experiences. Specifically, this study explores the perceptions of white adult mentors and black and Latino protégés of their activities, interactions, and their views on the advantages and drawbacks of their cross-race mentoring relationship.

This study explores mentors and protégés in cross-race mentoring relationships to grasp a better idea of the meanings each mentoring partner attaches to their mentoring 
experience. Respondents are sampled from one formal mentoring program at a four-year university. Nine mentors and eleven protégés are interviewed following a semi-structured format. From this sample of twenty participants, six complete pairs participated. The remaining seven respondents make up the supplemental data group, as their mentoring partner did not participate in this study.

This study suggests that the intergroup contact theory is useful in explaining the outcomes of these cross-race mentoring relationships. Not only does this Thesis support the intergroup contact theory, it also broadens our understanding by painting a more complete picture of how the optimal conditions emerge and work to strengthen and reinforce one another. Additionally, this research highlights how important understanding both the in-group and out-group member's perceptions are when exploring cross-race mentoring relationships and the intergroup contact theory. Finally, this study supports the notion that a mentoring program's structure is an important feature that can greatly enhance or inhibit mentoring bonds between mentors and protégés. 


\section{TABLE OF CONTENTS}

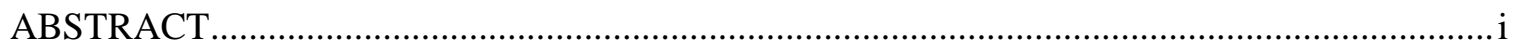

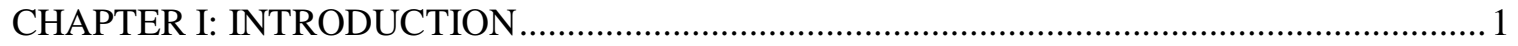

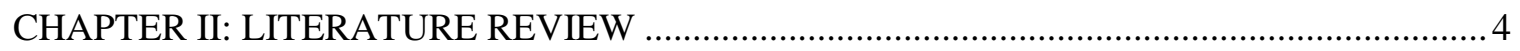

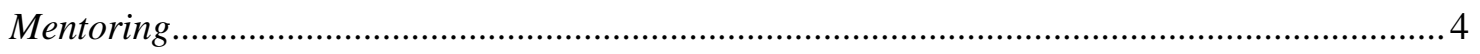

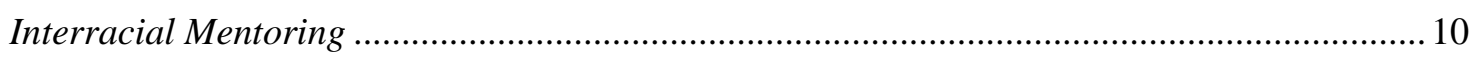

Considering the Forms of Contemporary Racism ............................................................... 14

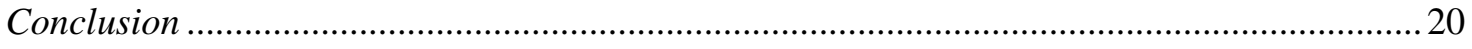

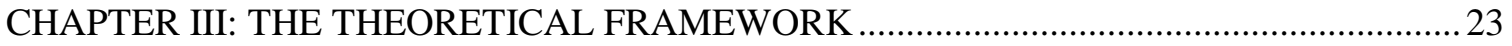

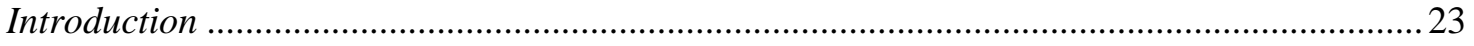

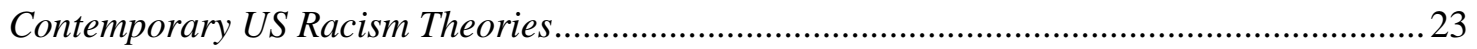

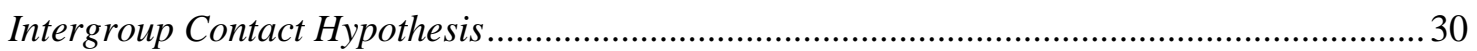

Additional Optimal Conditions in Light of Contemporary US Racism...................................... 35

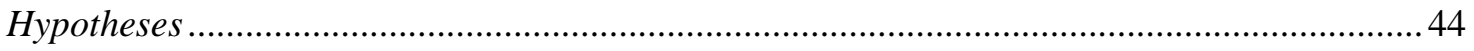

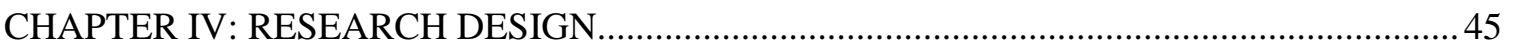

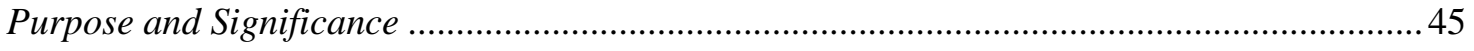

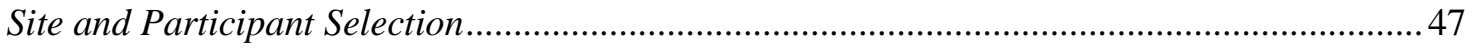

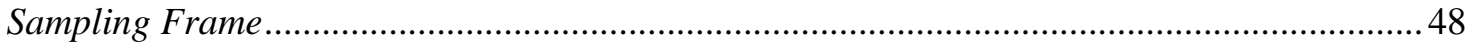

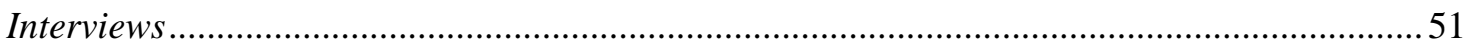

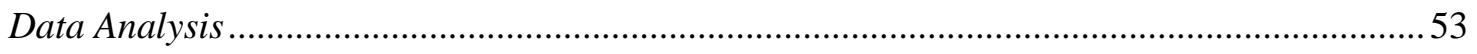

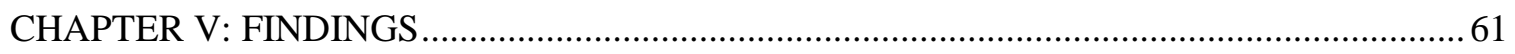

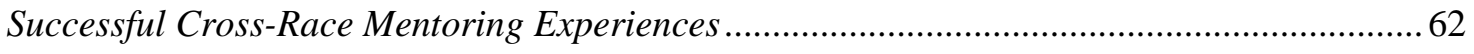

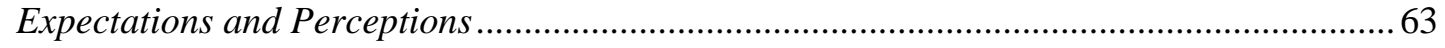

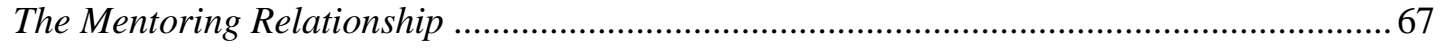

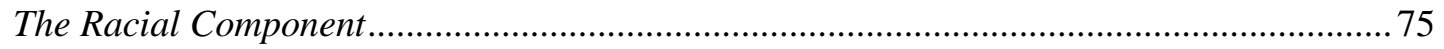

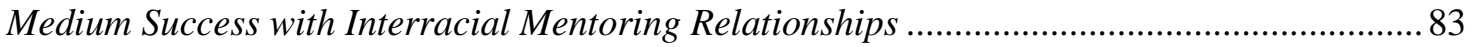




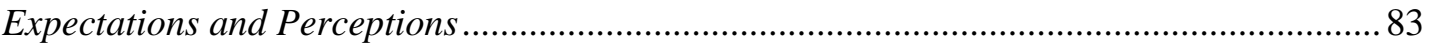

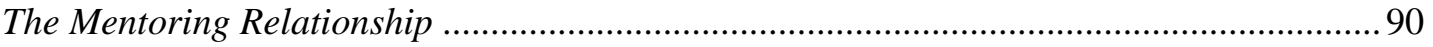

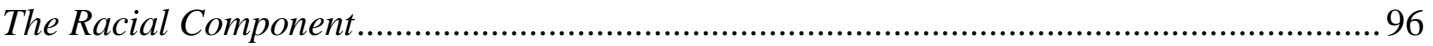

Low-to-No Success in Cross-Race Mentoring Relationships................................................. 102

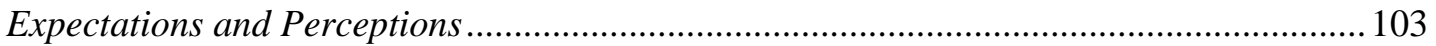

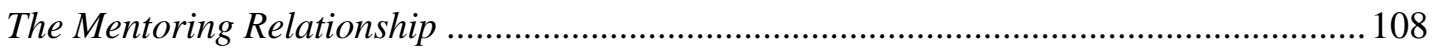

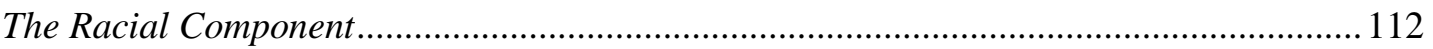

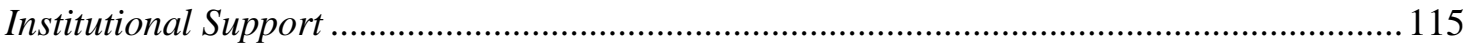

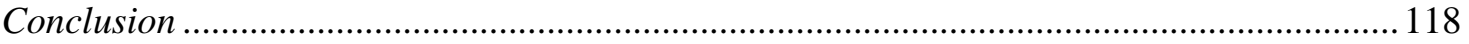

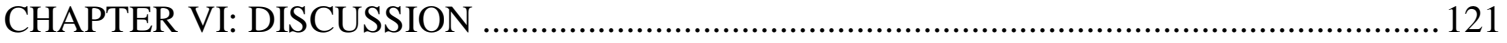

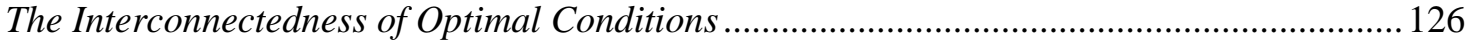

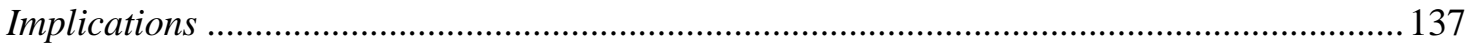

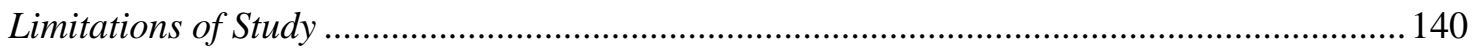

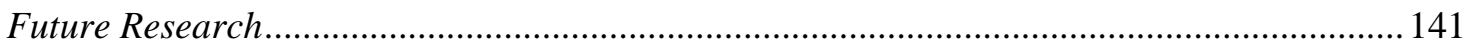

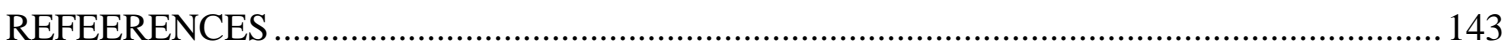

Appendix A: Invatation Letter for Protégés from New Horizons Mentoring Program ............... 151

Appendix B: Voluntary and Informed Consent for Protégé Participants .................................... 152

Appendix C: Voluntary and Informed Consent for Mentors ..................................................... 155

Appendix D: Interview Guide for Protégé Participants ............................................................... 157

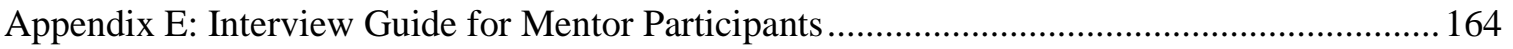




\section{LIST OF TABLES}

TABLE 1: Intergroup Contact Theory: A Model for Interracial Contact................43

TABLE 2: Conditions Present In Cross-Race Mentoring Relationships...............125 


\section{CHAPTER I: INTRODUCTION}

Cross-race mentoring relationships are of interest to the theory and practice of mentoring and they also speak to a longstanding problem in sociological study of prejudice. The mentoring literature reveals some disagreement regarding the advisability of cross-race matching for adolescent mentees. Some researchers stress same-race matching, while others emphasize the problem this creates for minorities facing a dearth of mentors. Sociologists and psychologists, on the other hand, have amassed evidence showing support for a contact hypothesis, which states frequent intergroup contact between equal-status members can lead to improved perceptions of the Other. However, to date, the contact hypothesis has not been explored in the context of mentoring relationships between white mentors and minority protégés.

The purpose of this thesis is to better understand the perceptions of mentors and protégés who are involved in cross-race mentoring relationships and asks does racism have to impede mentoring relationships between whites and blacks or between whites and Latinos, in the United States. It will focus on how interracial mentoring experiences are described by both mentors and protégés. I am particularly interested in exploring if and how the optimal conditions set forth by the intergroup contact theory emerge in this specific form of intergroup contact. On a broader scope, this Thesis can elucidate how individuals from different backgrounds develop strong connections and avoid prejudices.

This thesis examines cross-race mentoring relationships from a formal mentoring program within higher education. These interracial mentoring relationships involve a 
white faculty adult mentor and first-generation undergraduate students of color, limited to black and Latino protégés. I interviewed eleven protégés and nine mentors. Together there are six complete mentoring pairs and a supplemental data group that consists of two mentors and five protégés, as their mentoring partners were not interviewed. Data was gathered using in-depth interviews with a semi-structured format. The interviews focused on the participant's perceptions and descriptions of their mentoring experiences. Mentors and protégés were interviewed separately in order to elicit more candid and honest answers.

Chapter two reviews the literature on mentoring, which includes a discussion on mentoring in general, followed with a section on cross-race mentoring specifically. This is done to identify the benefits of mentoring and the conditions under which these benefits are likely to occur. This chapter also highlights how underdeveloped our knowledge is in the area of cross-race mentoring. Finally, this chapter reviews the literature on contemporary US racism to explain how interracial mentoring relationships might be impeded by this new and more subtle form of racism.

Chapter three delineates the theoretical framework that grounds this thesis. This chapter incorporates theories on contemporary racism and the intergroup contact theory. Following this I propose additional conditions to be added to the intergroup contact theory and layout hypotheses for the thesis.

Chapter four describes the methodological design of this thesis. This chapter outlines the research questions, participant selection and site, data instrument, and data 
analysis. This section ends with a description of how the optimal conditions were understood during the coding process.

Chapter five is a detailed overview of the major findings of this thesis. This section explicates how the optimal conditions emerged in the different mentoring pairs according to how successful their cross-race mentoring experience was.

Chapter six offers a more in-depth analysis of the significant findings. In this section the interconnectedness of the conditions are discussed. Additionally, this chapter highlights the study's contributions to the areas of mentoring and the intergroup contact theory. I then address the limitations of the study and propose directions for future research. 


\section{CHAPTER II: LITERATURE REVIEW}

To better understand why there is disagreement surrounding the advisability of cross-race matching in mentoring programs this chapter is spurred by two questions: (1) does contemporary US racism have to scuttle well-meaning cross-race mentoring interventions between white adult mentors and black proteges, and between whites and Latinos? (2) More specificially, what are the properties of contemporary racism that are likely to thwart cross-race mentoring relationships? This chapter reviews the literature where preliminary answers to these questions can be found. First, research on mentoring and the benefits likely to acrrue, in general, and the particular conditions that are found to engender these benefits. Second, an examination on the literature of cross-race mentoring, specifically. Finally, on contemporary US racism and its distinct features and forms.

\section{Mentoring}

This section intends to conceptualize mentoring and highlight the empirical evidence supporting the efficacy and contributions of mentoring relationships. In addition to this, I will identify the conditions found to engender the best results. While the concept and practice of mentoring is hardly a new phenomenon, it lacks a clear and unified definition and theory to undergird the study and development of mentoring programs. However, there are commonalities that emerge among the variegated conceptualizations associated with mentoring. In fact, a definition that is applicable to this study was developed by Anderson and Shannon (1988) during a comprehensive examination of the mentoring literature. They write: 
[Mentoring is] a nurturing process in which a more skilled or experienced person, serving as a role model, teaches, sponsors, encourages, counsels, and befriends a less skilled or less experienced person for the purpose of promoting the latter's professional and/or personal development. (Anderson and Shannon 1988:40)

Despite this concise definition, mentoring relationships do not neatly fit in a monolithic category (Anderson and Shannon 1988; Erickson et al. 2009; Hansman 2002; Jacobi 1991). The social context of the mentoring program sheds light on the specific functions it is meant to fulfill. For example, mentoring relationships can be informal or formal, and are often resources allocated for disadvantaged youth, academic retention, higher education success, and/or professional development. A commonality among mentorships across different social contexts is that it is a relationship between a more experienced member of the particular organization or setting and a less experienced member in the same setting that involves a supportive, skill-building, and advice giving dynamic (Campbell and Campbell 2007; Eby et al. 2008; Erickson et al. 2009; Jacobi 1991; Kram 1983).

The list of functions that mentorships are believed to serve is both extensive and, at times, ambiguous. Still, these functions most often fall into two broad categories: psychosocial functions or career/academic functions (Jacobi 1991; Kram 1983). Even this is not consistently agreed upon within the mentoring field, and Burke (1984) notes that role-modeling is a distinct function and fits neither in the psychosocial nor career function category. Psychosocial functions refer to the protégé's emotional well-being ${ }^{1}$ (Kram 1983) and are often the focus of youth mentoring programs (DuBois et al. 2002;

\footnotetext{
${ }^{1}$ Kram (1983) describes how the mentor provides psychosocial functions through advice-giving, positive reception-and-validation, role-modeling, and building a friendship. She explains that these functions serve to enhance the protégé's self-efficacy, capabilities, and self-confidence.
} 
Rhodes et al. 2006), but remain an integral feature of most mentorships, regardless of its environmental context (Ensher and Murphy 1997; Kram 1983; Santos and Reigadas 2002). Career/academic functions encompass organizational information and expertise passed from mentor to protégé as well as other benefits that enhance the mentee's career or academic endeavors, such as increasing the protégé's visibility within the organization and expanding their social network (Burke 1984; Kram 1983; Santos and Reigadas 2002). Thus, an ideal mentorship will involve functions that fit into all three of these categories.

Youth mentoring programs are credited for positively impacting youths' selfdevelopment, improving outside relationships with peers and parents (DuBois et al. 2002; Rhodes et al. 2002), increasing academic achievement (Diversi and Mecham 2005), decreasing criminal activity (Deutsch and Jones 2008; Rhodes et al. 2006), and lowering rates of substance abuse (Spencer 2006). Studies indicate that student-faculty mentoring programs in higher education provide significant benefits to students, particularly those considered at-risk because of academic difficulties (Campbell and Campbell 2007; RossThomas and Bryant 1994; Santos and Reigadas 2002). The benefits include improvements in self-efficacy, organization socialization (Santos and Reigadas 2002), increased academic scores, completing more credit units, and lower rates of dropping out (Campbell and Campbell 2007). However, it is unclear if these benefits sustain themselves over time (Campbell and Campbell 2007; Santos and Reigadas 2002).

An effective mentoring relationship is grounded in the belief that acknowledging and highlighting the mentee's strengths through a supportive interpersonal relationship 
with a more experienced individual promotes a healthier self image (DuBois et al. 2002; Reichert, Stoudt, and Kuriloff 2006; Rhodes et al. 2002), and can enhance academic and career success (Eby et al. 2008; Hansman 2002; Jacobi 1991; Ross-Thomas 1994). Thus, mentoring is often lauded as a compensatory resource, whether it be informal or formal, or in the context of youth (Diversi and Mecham 2005; Rhodes et al. 2006; Spencer 2006), higher education (Campbell and Campbell 2007; Ross-Thomas and Bryant 1994; Santos and Reigadas 2002), or the workforce (Chao and Gardner 1992; Kram 1983; Wilson, Valentine and, Pereira 2002). However, a closer inspection of the mentoring literature paints a more complicated picture.

A meta-analysis by Eby et al. (2008), which is one of the first studies to compare different contexts of mentorships with one another and to non-mentored individuals, finds positive correlations with mentoring and a variety of protégé outcomes. More specifically, the findings suggest that attitudinal outcomes have a stronger link to mentoring relationships than behavioral outcomes. Additionally, the authors indicate that academic mentoring involves a stronger relationship with mentee outcomes than either youth or workplace mentoring, with workplace mentoring falling in the middle. However, the researchers caution against overestimating the benefits of mentoring programs, as the effect size, while significant, is small (Eby et al. 2008). These empirical successes, while demonstrating the value in mentoring endeavors, are not guaranteed results; rather, they are potential outcomes, which must be facilitated by the mentor, protégé and a well developed program (DuBois et al. 2002; Spencer 2006). 
The conditions under which mentoring of young people leads to the best results include the development of a nurturing and trusting bond between the mentor and protégé (Diversi and Mecham 2005; Rhodes et al. 2006; Santos and Reigadas 2002; Spencer 2006). Additionally, the mentor's motivations for volunteering are important because it can determine how important the relationship will be to the mentor. For example, mentors who enter the relationship with the expectations of meeting new people, enhancing their own life, and contributing to their community as a whole are likely to foster an authentic and sustaining mentoring relationship (Grossman and Rhodes 2002). Thus, authenticity is a building a block when developing trust, and a core feature of a relationship with genuine empathy.

Researchers often highlight the presence of authenticity and empathy when trust develops in mentorships and strong interpersonal bonds are formed (Rhodes et al. 2006; Spencer 2006). Rhodes (2004) emphasizes the effectiveness for mentors and mentees to engage in activities outside of the academic realm to ensure strong interpersonal bonds. This is further supported by DuBois' et al. (2002) meta-analysis that suggests mentoring programs outside of the school environment produce stronger beneficial outcomes than those within schools. These studies emphasize how interpersonal bonds are strengthened through enjoyable activities (Rhodes 2004). Despite this, Eby et al. (2008) determines that academic mentorships yield more positive outcomes than mentorships serving at-risk youth and those within a workplace setting. The researchers posit that this could be a result of academic mentoring programs' more focused and specific goals (Eby et al. 2008) rather than an indication that closer interpersonal ties emerge within academic mentoring relationships. However, DuBois et al. (2002) discover that the benefits accrued 
from mentoring interventions are less dependent on the mentoring program's goals, model, and whether mentoring occurs in conjunction with additional services for the young protégés than specific features or practices of the mentoring program. The features that DuBois et al. (2002) propose are the most likely to engender protégé benefits include "ongoing training for mentors, structured activities for mentors and youth as well as expectations for frequency of contact, mechanisms for support and involvement of parents, and monitoring of overall program implementation" (p. 188).

Distinguishing between informal and formal mentorships provides some insight into the inconsistent findings and lack of agreement concerning the effectiveness of mentoring interventions. Formal mentoring relationships are deliberately created and matched, often with no clear criteria for why specific pairs are matched together (Chao and Gardner 1992). On the other hand, informal mentoring relationships develop organically and are rooted in strong interpersonal ties and common interests between mentor and protégé (Kram 1983). However, informal mentorships are difficult for minority students to access, particularly in historically white institutions (Jacobi 1991). In fact, Erickson et al. (2009) discover that informal mentoring relationships that develop between adults and young adults are more likely to widen social inequalities rather than reduce them. The researchers identify two contributing factors that help explain this phenomenon. First, youth and young adults with access to a wide range of social resources are the most likely to form informal mentorships. Secondly, the most advantaged young people (i.e., those from upper- and middle-class backgrounds) are the best equipped to capitalize on the benefits of these relationships (Erickson et al. 2009). This underscores the need for effective formal mentoring programs in universities that are 
designed to meet the needs of ethnic minority and first-generation students. However, if universities and colleges that are traditionally white institutions are to implement these types of mentoring interventions, it is likely that the majority of these mentoring relationships will comprise cross-race pairs. This suggests the need for a broader understanding of how interracial mentorships might evolve differently than same-race mentoring relationships.

\section{Interracial Mentoring}

This research aims to better understand the process of building interpersonal relationships in the context of interracial mentoring dyads, while potentially contributing to a more inclusive, integrated, and thus, healthier society. Throughout the United States, mentoring programs are developed to improve the lives of a diverse population (Campbell and Campbell 2007; DuBois et al. 2002; Hansman 2002; Jacobi 1991; Rhodes et al. 2006). However, there is a reasonable, ongoing debate on whether cross-race mentoring relationships, specifically between whites and blacks, can be as effective as same-race mentoring relationships. Both proponents and opponents express valid reasons for their position.

Those who express concern about cross-race matches speak to the potential for white mentors to distort the protégé's racial/ethnic identity, whether consciously or unconsciously (Rhodes et al. 2002). For example, a white mentor may promote their own racial views and encourage their mentee to assimilate into the white mainstream. This can happen without the mentor's awareness or even motivation to do so. Simply avoiding difficult conversations about racial issues or downplaying the significance of race can 
potentially be interpreted by a young person of color that experiences of racial

discrimination are unimportant, or even that non-white culture is somehow un-American (Riechert et al. 2006; Robinson and Ward 1991). Additionally, white Americans may experience discomfort during discussions centered on racial issues.

Some have argued that middle-class European Americans might experience powerful negative emotions (e.g., guilt and defensiveness) in relation to America's history of racial oppression and that such emotions are likely to impede the development of mentor relationships and dampen motivation for continued multi-cultural learning (Rhodes et al. 2002:2116).

Furthermore, there are concerns that community solidarity is compromised when mentees of color are matched with white mentors (Robinson and Ward 1991; Rhodes 2002). More specifically, there is uncertainty about the message this promotes to the minority protégé. For example, this might be interpreted as an indication that positive role models are unavailable from communities of color (Rhodes et al. 2002; Santos and Reigadas 2002). However, this position on the advisability of race matching mentoring dyads is more concerned with determining if cross-race mentoring interventions work as well as same-race mentoring, rather than developing mentoring practices that can better support these forms of mentoring relationships. This greatly limits our understanding of, and how we evaluate cross-race mentoring relationships.

Conversely, those on other side of this conversation acknowledge that there are currently more people of color in need of mentors than there are minority mentors (Hansman 2002; Rhodes 2002; Rhodes et al. 2002; Spencer 2006); and thus, understand that waiting for a same-race match can result in valuable time lost for the protégé (Diversi and Mecham 2005; Rhodes et al. 2002; Spencer 2006). Therefore, these 
proponents identify practices that can work to overcome the obstacles of different lived experiences. For instance, it is important to select mentors who promote their mentee's cultural and ethnic identity (Diversi and Mecham 2005; Deutsch and Jones 2008; Rhodes et al. 2002) and who remain cognizant of the lived experience of minorities in the United States (Rhodes et al. 2002; Robinson and Ward 1991; Sellers et al. 2006). Moreover, research indicates that other factors may be more significant than the mentor's and mentee's race or ethnicity, such as parental support/disapproval (DuBois et al. 2002; Rhodes et al. 2002), individual personality traits (Diversi and Mecham 2005; Spencer 2006), criteria for matching (Campbell and Campbell 2007; Chao and Gardner 1992; Kram 1983), and gender (Hansman 2002; Ensher and Murphy 1997; Rhodes et al. 2002). Lastly, proponents also bring attention to the potential of reducing social distance between members of different racial and ethnic groups (Diversi and Mecham 2005; Rhodes et al. 2002). Thus, these relationships hold the potential to benefit the mentee, the mentor, and society in general.

Within the mentoring literature there is a dearth of studies that focus primarily on interracial mentoring relationships. Despite the growing demand to explore interracial mentoring relationships, most of these studies remain focused on protégé outcomes. For example, Campbell and Campbell (2007) investigated a college mentoring program consisting mainly of cross-race dyads, but concentrated the findings on protégé effects. They discovered that those students in ethnically matched mentorships enrolled and completed more credit units, achieved higher GPAs, increased in graduation rates, and were more likely to enroll in graduate programs at the same university than students in cross-ethnic mentorships (Campbell and Campbell 2007). Similarly, Santos and Reigadas 
(2002) find that Latino college student protégés who were ethnically matched with their mentor held more positive perceptions of their mentor's helpfulness in enhancing their own development and clarifying their academic and career goals than non-matched Latino protégés. Additionally, Santos and Reigadas (2002) discovered that the rolemodeling function emerged more frequently within same-ethnic matched mentorships than cross-race pairs. They argue "that a Latino mentor was a more salient and identifiable role model for [Latino] mentees, where similarities in values, expectations, and background enhanced the perceived supportiveness and benefits of the relationship" (Santos and Reigadas 2002:47).On the other hand, regardless of whether mentored students were ethnically matched, mentoring was associated with protégé benefits (Campbell and Campbell 2007; Santos and Reigadas 2002). Furthermore, DuBois' et al. (2002) revealed in their more rigorous meta-analysis that race and ethnicity were not significant factors for explaining which mentoring programs produced the desired benefits for protégé youth. Instead, mentoring program practices and procedures emerged as the characteristics that produced the greatest impact on protégé outcomes. Together these studies suggest that cross-race mentoring interventions can experience barriers that are unique to the interethnic nature of these dyads (Campbell and Campbell 2007; Santos and Reigadas 2002). However, if the mentoring program implements the most effective practices and procedures (i.e., structured activities for mentoring pairs, on-going training and support, strong emotional connections develop, etc.) they are just as capable of producing protégé benefits to similarly structured same-race mentoring interventions (DuBois et al. 2002). 


\section{Considering the Forms of Contemporary Racism}

This section reviews race relations in the United States to situate interracial mentorships within a sociological context, which highlights the racial dynamics and provides an interdisciplinary framework for the analysis of cross-race mentoring relationships. There is no reason to assume that racial attitudes play out differently within mentoring relationships than in society in general; mentorships do not exist in a vacuum. Both mentor and protégé bring with them their own racial attitudes and experiences upon entering a cross-race mentorship. Therefore, this section explores the following question: In light of social science, what are the properties of contemporary racism that are likely to foul mentoring relationships between white mentors and minority youth and young adults?

The insidious nature of contemporary racism is that it lies just "under the surface" for whites, yet produces serious life consequences for minorities (Bobo 2001). Contemporary anti-black racism, variously labeled color-blind racism (Bonilla-Silva 2003), silent racism (Trepagnier 2010), everyday racism (Zamudio and Rios 2006), laissez-faire racism (Bobo 2001), and implicit racial biases (Dovidio et al.2002), is characterized by its more subtle and covert nature, as compared to racism prior to the Civil Rights era. While the above mentioned forms of racism involve nuances that make each type distinct, they all represent the new era of racial oppression in the United States, in which racial inequalities remain a distinct feature of our society but are often discussed in nonracial terms (i.e., economic, political, cultural etc.) by the majority of white Americans (Bobo 2001; Bonilla-Silva 2003), a point further discussed later in this section. It is not uncommon for white Americans to explain racial inequality without any 
mention of our racist history and its continuing impacts on minorities' life outcomes through discriminatory practices that have become institutionalized (Bonilla-Silva 2003; Feagin 2001; Trepagnier 2010). In fact, one of the most deleterious features of contemporary racism is its ability to bypass the link between racist behavior and a conscious dislike or prejudice on the actor's part.

The issue of whites' racial attitudes is complex and underscores the longstanding debate on the prevalence of racism and discrimination in the United States. Admitting one's own racial prejudices is no longer socially acceptable, as most Americans now hold strong egalitarian views; therefore, race and racism are frequently avoided topics among white Americans (Bonilla-Silva 2003; Jenson 2005; Trepagnier 2010). It is precisely when questions move beyond explicit racial attitudes and begin to examine the more implicit and unconscious understandings white Americans hold about racial issues that their racial attitudes become increasingly complex and, at times, contradictory (Bobo 2001; Bonilla-Silva 2003; Dovidio et al. 2002; Feagin 2001; Trepagnier 2010). Sadly, an egalitarian and inclusive ideology has not transferred into the daily practices for the majority of Americans (Bobo 2001).Racism persists, among other areas, in our society's increasing levels of residential and school segregation (Braddock and Gonzalez 2010; Massey 2004), in job markets (Feagin 2001), and in the workings of law enforcement (Alexander 2010).

Although the United States is currently more ethnically diverse than ever in its history, Braddock and Gonzalez (2010) inform us that schools and neighborhoods are increasing in racial and ethnic segregation. Massey's (2004) examination of residential 
trends reflects this pattern of segregation; he finds that 60 percent of Black Americans live in highly segregated neighborhoods and 40 percent live under hypersegregated ${ }^{2}$ conditions. Additionally, school segregation statistics indicate a pattern towards resegregation (Berends and Peñaloza 2010; Braddock and Gonzalez 2010). For example, "In 2004, the average white student attended a school that was about 80 [percent] white; the average black student attended a school that was 34 [percent] white; and the average Latino student attended a school that was 28 [percent] white" (Berends and Peñaloza 2010:979).

School segregation perpetuates racial inequality and stifles social cohesion. Braddock and Gonzalez (2010) write that "because of their role in developing tolerance, respect for diverse others, a common identity, and shared values, schools have long been recognized as key institutions affecting social cohesion” (p. 1634). School and neighborhood segregation consolidates those with access to resources into one area and those with insufficient access into another. For example, minority segregated schools tend to be situated in areas with low levels of family socioeconomic status (Berends and Peñaloza 2010) and lack resources, staff, and curriculum that are on par with predominately white schools (Goldsmith 2004; Massey 1993). This situation undermines black and Latino students' academic achievement. Higher rates of pro-school attitudes found among these students cannot make up for the deficit in school resources and rigor

\footnotetext{
${ }^{2}$ Massey (1993) explains that residential segregation is often assessed by examining five dimensions of geographic traits. For example, segregation can lead to varying levels of unevenness and racial isolation. Additionally, it can produce racially clustered enclaves or they can be concentrated in small spaces. Lastly, segregation can result in minority groups that are spatially centralized on the periphery of a city or within the urban core. "A high score on any single dimension is serious because it removes blacks from full participation in urban society and limits their access to its benefits. As segregation accumulates across multiple dimensions," hypersegregation emerges (P.74).
} 
(Downey, Ainsworth, and Qian 2009; Goldsmith 2004). Segregated socialization in school and community heighten and reinforce each groups' hierarchical social position. In this sense, racial and ethnic isolation become self-sustaining by limiting the students' opportunities to cultivate relationships that transcend racial, nationality, and class boundaries (Beach and Sernhede 2011), which significantly diminishes their likelihood of engaging in cross-race and cross-class relationships as adults (Braddock and Gonzalez 2010). This prevents the inclusion and integration of individuals with contrasting backgrounds, knowledge, habits, and ideas (Beach and Sernhede 2011). Braddock and Gonzalez (2010) argue that while both school and residential segregation significantly influence later life preferences, school segregation produces a considerably stronger affect. This indicates that group boundaries grow increasingly rigid and insurmountable throughout school socialization in segregated environments.

Growing up in environments of residential and school segregation significantly impacts adult choices and preferences, particularly in relation to social networks (Beach and Sernhede 2011; Braddock and Gonzalez 2010), living areas (Bobo 2001; BonillaSilva and Embrick 2007), and perceptions of racial inequality (Bonilla-Silva 2003; Trepagnier 2010). For example, Braddock and Gonzalez (2010) discover that minority individuals who attend segregated schools and live in segregated neighborhoods are more likely than their counterparts to prefer same-race friends and neighborhoods, and feel the greatest social distance from the mainstream white population. Similarly, white students attending highly segregated schools and living in segregated communities are more likely than their counterparts to prefer same-race networks, neighborhoods, and tend to work in 
segregated professions (Braddock and Gonzalez 2010). Thus, the lack of diversity perpetuates segregated lifestyles.

Residential and school segregation also impedes the development of crosscultural friendships and places barriers during interracial communication, particularly at the interpersonal level. Riegle-Crumb and Callahan (2009) emphasize the importance of social networks, particularly during adolescence, that offer information and access to resources; however, segregated lifestyles impede the development of social connections between whites and blacks (and between whites and other people of color) (Bonilla-Silva and Embrick 2007; Braddock and Gonzalez 2010; Massey 2004; Riegle-Crumb and Callahan 2009). Although research indicates that friendships can and do emerge across racial and ethnic groups, most Americans experience a paucity of opportunities to forge these types of friendships (Braddock and Gonzalez 2010; Riegle-Crumb and Callahan 2009).

Contemporary racism within interpersonal interactions emerges in a variety of ways, including when white Americans minimize or even deny institutional racism and its significance (Bonilla-Silva 2003) or when whites treat blacks and Latinos with suspicion (Alforo 2009; Sellers et al. 2006) because of stereotypical images (Trepagnier 2010). There seems to be a clear link between residential and school segregation and the salience of stereotypes. For the majority of white Americans, particularly those living in majority-white areas, stereotypes about black Americans are often their main source of knowledge and therefore frame whites' interpretations of interactions with and the behaviors of black Americans (Feagin 2001), as well as other people of color. These 
stereotypes are ubiquitous in our society and are constructed by the media (Trepagnier 2010), political discourse (Feagin 2001), and during interpersonal communication between whites (Bonilla-Silva 2003). Thus, the institutional features of contemporary racism (i.e., segregation and racial inequality) foster miscommunication and distrust during interracial interactions and leave few opportunities for white Americans to examine their privilege and the exclusion of Americans of color.

Before an individual of color can reconcile an interaction with (or behaviors of) a white person, they must determine if it is a consequence of racial bias (Reichert et al. 2006; Sellers et al. 2006). Because discrimination today often resembles more subtle forms of racism rather than blatant racial epithets, as was the case during the Jim Crow era, it demands more cognitive effort (these subtle forms are discussed in more detail further ahead). For this reason, the psychological effects are significant for minority adolescents and young adults, who spend valuable time mentally processing new and covert forms of discrimination. The adverse psychological effects of perceived discrimination include lower self-esteem, decreased motivation for academic achievement, anger, behavioral problems, and lower levels of life satisfaction (Alfaro et al. 2009; Reichert et al. 2006; Sellers et al. 2006). The concept of perceived discrimination refers to an individual's interpretation of an interaction or action as motivated by racism or prejudice. Thus, whether the actor consciously acted in a racist manner is irrelevant; rather, this concept recognizes that regardless of the intent, the consequences are real and powerful (Alfaro et al. 2009; Sellers et al. 2006). The daily experience for Americans of color is overloaded with the need for interpreting the world through a racial lens (Sellers et al. 2006). 
Dovidio et al. (2002) indicate that black Americans depend on nonverbal cues to assess white Americans' racial biases and are often correct in their interpretations. Sellers et al. (2006) discover that black adolescents who think other racial groups hold negative beliefs about blacks are more likely to experience racial discrimination. However, these same beliefs serve to protect them from the psychological impacts of racial discrimination. This suggests that those who expect, and thus, experience higher rates of discrimination are more effective at developing coping strategies to combat the psychological effects of discrimination (Sellers et al. 2006). At the same time, it implies that these individuals are more critical and find it difficult to develop trust for others outside of their group, specifically in respect to white Americans.

To recap, patterns of racial segregation in the United States continue to be a fixture of our society, significantly limiting social cohesion; residential and school segregation are significant factors for current racial inequality and the prevalence of racial stereotypes; contemporary racism is qualitatively distinct from traditional racism and is characterized by its more subtle and often unconscious forms; and Americans of color continue to be negatively impacted by racial discrimination and are forced to examine their daily experiences through a racial lens.

\section{Conclusion}

This brief review of the literature on mentoring highlights the wide range of functions mentoring interventions are designed to serve and point to a variety of outcomes that can arise depending on the context, goals, and structure of the mentoring program. Positive outcomes for mentoring relationships are greatly enhanced when the 
mentoring program has a solid infrastructure to provide on-going training and support for their mentors. Additionally, when mentoring programs facilitate specific activities for mentoring pairs to engage in the mentor and protégé are more likely to develop a close personal bond. The mentor's motivations and goals for deciding to be a mentor is also another important factor that affects the mentoring relationship. Mentors who become mentors to help another individual and contribute to the larger community tend to build beneficial mentoring relationships.

There are few studies that focus specifically on cross-race mentoring, but from the few that have it is found that cross-race mentoring interventions do produce beneficial outcomes. While there is still disagreement on if same-race matching engenders more benefits than cross-race matching, there are researchers who have discovered that race and ethnicity are not significant in determining the outcome of mentoring experiences. Rather, it is the structure of the program and the mentoring practices that enhance or impede successful mentoring relationships. However, lacking from this area of the mentoring literature is a more in-depth understanding of how cross-race mentoring relationships develop and how white mentors and protégés of color perceive their relationships.

The sociological literature on race relations and contemporary racism in the US indicates that racial segregation continues to plague our society and works to prevent social cohesion; maintain racial inequality; and leaves little chance for white Americans to challenge stereotypes about racial minorities. While many whites believe racism is a thing of the past, Americans of color continue to feel the impacts of racial discrimination 
and understand that racism has not disappeared; it has taken new and covert forms. This points to questions not explored in the mentoring literature about some of the obstacles and barriers that are unique to cross-race matches. Thus, the next chapter will discuss contemporary racism in more detail and outline how the intergroup contact theory might be applied to the context of interracial mentoring relationships. 


\section{CHAPTER III: THE THEORETICAL FRAMEWORK}

\section{Introduction}

This chapter incorporates the theoretical contributions from research on contemporary racism and the intergroup contact hypothesis in order to propose a new model of optimal conditions for intergroup contact in the context of interracial mentoring. This section is organized according to: (1) theories of contemporary racism (2) Allport's original intergroup contact hypothesis and recent contributions to the theory, and (3) additional optimal conditions gleaned from contemporary US racism theories. Following this, I propose a refined intergroup contact theory that integrates Allport's original premise, subsequent intergroup contact research, and the additions from contemporary racism.

\section{Contemporary US Racism Theories}

Social scientists continue to develop and refine concepts and theories that address contemporary U.S. racism. Below is a synthesis of some of the concepts and theories that seem the most applicable to how contemporary racism might frustrate well-meaning efforts of cross-race mentoring interventions. In the United States we stand at a cross road when it comes to race relations; a significant majority of white Americans explicitly reject racism and acknowledge how wrong it is to prejudge someone based on the color of one's skin; however, experiences of racism are neither rare nor insignificant in the daily lives of Americans of color (Bobo 2001). How then do social scientists explain this racial paradox? Certainly, there is no one answer that can possibly unravel this complex puzzle without engendering new questions. Despite this, there are a number of researchers in the disciplines ofpsychology and sociology who attempt to explicate this 
paradox. These researchers and the concepts they develop and refine have implications for the study of interracial mentoring relationships.

In the Post-Civil Rights era, racism is now understood as involving both an overt, conscious component and a more subtle (Bonilla-Silva 2003; Feagin 2001) and even unconscious aspect (Dovidio et al. 2002; Trepagnier 2001, 2010). Dovidio et al. have corroborated that implicit racial attitudes significantly impact interracial communication and that these attitudes can often conflict with one's explicit racial attitudes. Explicit attitudes are found to predict verbal (or conscious) behaviors, while implicit attitudes impact nonverbal (unconscious) behaviors, which make it difficult for people to control these types of cues (Dovidio et al. 2002). During interracial interactions, white individuals use their explicit racial attitudes to evaluate how friendly they act during the interaction; whereas, black individuals rely on whites' nonverbal cues to evaluate the white individual's friendliness (Dovidio et al. 2002). Thus, interpretations of interracial interactions depend as much on the content of the interactions as on the historical narrative and subjective perspectives each actor bring with them. Moreover, this highlights how racism can be perceived even when the actor does not consciously intend to behave in a racist manner. This work indicates that negative racial attitudes can lie below the surface of whites' awareness; that these implicit racial attitudes can be measured; and that racial Others are adept at picking up on the subtle features of implicit racial attitudes (Dovidio et al. 2002). While this research underscores the potential for miscommunication during interracial interactions, it does not address the question of how and why these implicit attitudes are formed. 
Bonilla-Silva (2003) and Trepagnier (2010) attempt to solve a piece of this racial puzzle; how white Americans can explicitly reject racism while simultaneously maintaining race privilege. Their sociological insights, however, can only be one piece of a much larger and complicated story. Despite this, these two theories help us better understand how whites think about race issues and identify coping techniques used by whites during uncomfortable interracial situations.

Bonilla-Silva (2003) delineates different ways white Americans think about and understand our social reality, and how this engenders new strategies for explicating the racial inequalities within the United States. He identifies four frames that frequently shape whites racial understandings. These frames, abstract liberalism, naturalization, cultural racism, and minimization of racism, provide ways of legitimizing the racial status quo without using the reasoning of scientific racism, which dominated the discourse prior to the Civil Rights era (Bonilla-Silva 2003; Feagin 2001). Bonilla-Silva (2003) distinguished between the four frames but notes that the frames often work in conjunction with one another.

The abstract liberalism frame is closely linked to individualistic ways of thinking that ignore the structural and institutional circumstances that significantly impact people's life outcomes. The naturalization frame often emerges in discussions on segregation and claims it is natural for people to choose living areas with residents that are similar to one's self, implying that racial segregation is not a result of racial discrimination. Similar to abstract liberalism, the naturalization frame also ignores structural causes contributing to residential and school segregation and the impact it has 
on life outcomes. The cultural racism frame looks to white culture as a reason for whites' successes and laments that black culture does not value economic and educational success in the same fashion and instead instills a culture of poverty (Bonilla-Silva 2003; Massey and Denton 1993). In essence, this frame blames the victim for their economic and social position and often uses explanations of a lack of effort or laziness for minorities' life outcomes (Bonilla-Silva 2003). Finally, the minimization of racism works just as its name implies. This frame emerges in statements such as "playing the race card" or "racism is a thing of the past" (Bonilla-Silva 2003). Thus, this frame assumes that racism only manifests in the form of overt derogatory statements from whites. These frames or ways of thinking are coined color-blind racism, as they are cultural tools whites use to legitimize and explain racial inequality without naming race as factor (Bonilla-Silva 2003). They are mechanisms for whites to confidently reject racist behaviors while providing whites with the rhetoric to "blame the victims" and rescind the historical impact racism continues to have on people of color in the United States (Bonilla-Silva 2003).

Trepagnier's $(2001,2010)$ analysis of contemporary racism examines how wellmeaning white Americans cope with uncomfortable racial issues and experiences. Her theory of silent racism underscores how subtle and unconscious racism is today. Trepagnier (2001) discovers that racism arises in subtle forms even from whites who reject the color-blind approach to race matters. She argues that too often whites who consider themselves to be not racist do not question their own racial attitudes. Silent racism does not refer to racist actions and behaviors; 
[Rather it] refers to the negative thoughts and beliefs that fuel everyday [and color-blind] racism and other racist action. Silent racism stems from the racist ideology that permeates U.S. society and inhabits the minds of all white people...it refers to the shared images and assumptions of members of the dominant group about the subordinate group...The images and assumptions derive from the dominant ideology, which is fluid and piecemeal " (Trepagnier 2010:15).

Silent racism is not a consequence of racial enmity; rather, silent racism is a symptom of our racist legacy and its lasting impacts that continue to go unquestioned; silent racism is a product of the cultural information that one cannot escape in the U.S. (Trepagnier 2010). Cultural information is a broad concept that refers to socially created knowledge, which is sustained as it transfers from one generation to the next throughout the socialization process (Johnson 2000). In the context that Trepagnier (2010) uses the term, it denotes information or messages that are disseminated through social outlets, such as the media and school system, and shapes our understandings of racial issues. For a significant portion of white Americans, particularly those living in and attending highly segregated areas and schools, the cultural information pertaining to black Americans (and Americans of color in general) is replete with misinformation that is negatively framed (Feagin 2000; Trepagnier 2001, 2010). This racial information is often unexamined, accepted and transmitted among whites as it bolsters whites' group position at the top of the racial hierarchy (Feagin 2001; Trepagnier 2010). This is not to claim that all whites are consciously protecting race privilege when the negative racial information is unquestioned, but to highlight the ease in which this racial information can lie just below the surface from whites' awareness. Trepagnier's $(2001,2010)$ research suggests that cultural racial information affects whites' racial understandings, even those with a high level of race awareness. Still, Trepagnier is able to identify the principle attributes of that 
subgroup of whites who can overcome the handicaps of silent racism. This is the part of her work that I feel is the most fitting to address the question: what type of white adult would make an effective mentor in an interracial mentoring relationship?

For white individuals, a high level of race awareness includes three components: (1) acknowledging the impacts of institutional racism and how it contributes to white privilege; (2) recognizing and comprehending the historical legacy of racism in the U.S.; and (3) remaining cognizant of one's own racist thoughts and behaviors (Trepagnier 2010). But—and this is the key to silent racism—even being aware of how racism plays out today does not preclude whites from internalizing the racist information that is ubiquitous in U.S. culture; no one is immune to cultural information (Trepagnier 2010). However, cultural information does not affect every person in the same way. Still, without a high level of race awareness whites are unlikely to question and recognize institutional and color-blind racism (Bonilla-Silva 2003). Whites with high levels of race awareness are not overly concerned of the label "racist", because they understand that inevitably every white person will at one time or another say something that can be perceived as racist. Trepagnier (2010) highlights how effective meaningful conversations between whites and people of color, particularly when the relationship between the two is a close one, about racial issues and racism can be for increasing whites' racial awareness.

In order for white Americans to participate in meaningful conversations that are able to cross racial barriers and facilitate mutual understanding, they not only have to be self-reflective, but also reject the notion that racism is limited to individual traits, which suggests the culpability lies solely in personal attitudes and behaviors. Jensen (2005) 
explains that many white Americans fail to thoroughly examine, and thus, understand the intersection of race, ethnicity, and life outcomes. He credits fear, guilt, and shame for contributing to the color-blind ideology that Bonilla-Silva identifies. Jensen (2005) agrees that a binary perception of racism prevents white Americans from acknowledging institutional racism. The desire to be perceived as not-racist often hinders even wellmeaning white people from recognizing the consequences of the racial hierarchy minorities confront on a daily basis. The fear of coming across as racist deters many white Americans from engaging in critical self-analysis, and instead, conditions many to suppress any negative beliefs and attitudes (Jensen 2005; Trepagnier 2010). This leaves little chance for a thoughtful analysis of how these attitudes and beliefs might affect one's behaviors.

In sum, contemporary racism emerges in more subtle forms than traditional overt racial bigotry; and, whites can and do engage in racists practices without a malicious intent or even being aware that their actions and behaviors are racist. Thus, in contrast to same-race mentorships, an interracial one must negotiate racial boundaries, which includes contemporary racism and different lived experiences, to establish an enduring and beneficial relationship. There is the chance that an individual of color may struggle to develop trust in the relationship, especially if their expectations and perceptions are based on limited interactions with whites. Simply putting people of different races and ethnicities into the same social environment does not guarantee mutual understanding. Therefore, to better understand how positive intergroup relations may emerge within cross-race mentorships the subsequent section will introduce and discuss the intergroup contact hypothesis. 


\section{Intergroup Contact Hypothesis}

A theory of the conditions under which interracial mentorships might be able to overcome the handicaps created by racism might usefully begin with Gordon Allport's intergroup contact hypothesis ( $\mathrm{ICH})$. While this theory highlights the potential of interpersonal contact between in- and out-group members to ameliorate in-group biases and out-group prejudices (Allport 1954), there remain gaps in the theory that if addressed could broaden our understanding of intergroup relations, generally, and more specifically, of interracial mentoring relationships. Therefore, this section includes a discussion of Allport's ICH, followed with a review of more current ICH research to identify how the theory has been expanded upon, and finally a conversation about how this research might further refine this theory.

Allport (1954) understands that contact takes a variety of forms, under a wide range of circumstances; thus, when he explains the positive effect intergroup contact can make on intergroup attitudes he is referring to specific types of interpersonal contact that meet particular conditions. Contact, in and of itself, does not necessitate a decline in prejudice, and in fact, under adversarial conditions serves to increase prejudices and enmity between groups. Before one can determine if intergroup contact is capable of establishing the optimal conditions that engender a reduction in negative out-group attitudes one must identify the nature of the contact (Allport 1954). For example, casual contact, which is not meaningful to the participants, is not likely to mitigate in-group biases or challenge out-group prejudices. Therefore, Allport (1954) delineates important variables that determine the quality of the contact, which in turn indicates if the optimal conditions for reducing prejudiced thinking are likely to emerge within the contact: (1) 
the amount of time spent interacting and the regularity of the contact; (2) the social status of the out-group in general, and specifically, the social status of the out-group member; (3) the assigned roles associated within the interaction; (4) the social environment that structures the intergroup contact; and (5) certain personality characteristics, such as level and nature of prejudice and personal life security (p.262). Once the nature of the contact is known, the next step is ensuring that the optimal conditions are present within the contact situation for it to be capable of reducing prejudice. These conditions are: (1) frequent contact, (2) authoritative or institutional support, (3) the support from above ensures equal-status, and (4) the individuals cooperate in order to reach a shared goal (Allport 1954:281). At the same time, Allport recognizes that this list of variables and optimal conditions is neither exhaustive nor a guarantee that prejudice will diminish; rather, it is a starting point for creating and fostering contact situations that can overcome in-group biases and out-group prejudices.

The inception of the contact hypothesis has spawned literally hundreds of studies examining it and the results of these studies have significantly refined our understanding of the ICH (Pettigrew and Tropp 2006). Pettigrew and Tropp (2006) undertook a comprehensive meta-analysis of the body of ICH research, which evaluated 515 studies, and what Hewstone and Swart (2011) call "the most convincing evidence accumulated" (p. 375) to date in support for the ICH. Pettigrew and Tropp's (2006) research stands out because of the methodological rigor used to evaluate research on Allport's hypothesis. For example, to address the question of participant selection bias, the researchers established a measurement to identify studies that severely limited the choice participants had for engaging in intergroup contact. This was done in an attempt to answer the 
question: is intergroup contact the mediator for reducing out-group prejudice attitudes or do people with the highest levels of prejudice (and therefore the least likely to change their attitudes) avoid intergroup contact? Pettigrew and Tropp (2006) also circumvent the problem ofpublication bias, the possibility that many empirically sound studies do not get published because:

Researchers may be reluctant to send in studies with modest or countertheory findings. And journals may publish studies with large effects and reject studies with small or no effects. Thus, reviews may systematically overestimate effect sizes, as they rely heavily on published work (Pettigrew and Tropp 2006:754).

This meta-analysis also probes the issue of generalizability to determine if and how a reduction in prejudice attitudes are generalized to other contact situations, out-group members, and/or out-groups not involved in the contact (Pettigrew and Tropp 2006).

Pettigrew and Tropp's (2006) meta-analysis found overwhelming support for Allport's ICH while also providing new insights for refining the ICH. They find a significant correlation between intergroup contact and diminishing levels of prejudiced attitudes, with 94 percent of the 515 studies included in the analysis revealing a significant and negative relationship between intergroup contact and prejudice. Of note isthe studies with the most methodological rigor yield the largest effect sizes (Pettigrew and Tropp 2006). Further supporting Allport's work the researchers explain how the optimal conditions are instrumental in facilitating positive outcomes for intergroup contact. These conditions significantly enhance contact and provide a useful framework for creating intergroup contact situations to ameliorate prejudice and for analyzing intergroup contact. Allport's condition of institutional support emerges as the most critical condition for promoting positive outcomes (Pettigrew and Tropp 2006). Still, the 
researchers strongly caution against relying on this condition in isolation (or any of the conditions in isolation). Rather, this condition is understood to be significant because of its ability to reinforce and strengthen the remaining conditions, particularly the conditions of ensuring equal-status and cooperating towards a shared goal. "Thus, consistent with Allport's original contentions,... optimal conditions for contact are best conceptualized as functioning together to facilitate positive intergroup outcomes rather than as entirely separate factors" (Pettigrew and Tropp 2006:766).

This study did more than just support the intergroup contact hypothesis; it also expanded the theory by confirming new conditions and clarifying unresolved issues. Pettigrew and Tropp (2006) found that intergroup contact's relationship with prejudice is not limited to racial and ethnic groups; it can also be applied to other out-groups, such as religious as political groups and out-groups tied to sexual identity. The researchers also discovered that mutual liking is central to the process that allows intergroup contact to diminish out-group biases. Additionally, group salience emerged as an important factor moderating generalizations of out-group attitudes (Pettigrew and Tropp 2006), further confirming prior ICH research that examines the role of group saliency (Van Oudenhoven, Groenewoud, and Hewstone 1996). When group saliency is high the individuals in the contact remain cognizant of one another's group membership and perceive the situation as intergroup contact, not merely interpersonal contact. "This means, essentially, that ingroup members who have contact with outgroup members, must at some level, continue to be aware of the contact partner as a member of the outgroup and not simply a positive individual" (Van Oudenhoven et al. 1996:658). This allows out-group prejudices to be challenged through positive intergroup contact and for 
these positive attitudes to be applied and generalized to the entire out-group (Hewstone and Brown 1986; Pettigrew and Tropp 2006; Van Oudenhoven et al. 1996).

Also adding to Allport's model of optimal conditions that promote intergroup contact's potential to reduce out-group prejudice, Van Dick et al. (2004) explore the relationship between perceived importance of contact and the positive effect intergroup contact can produce on out-group attitudes. Following Allport's (1954) lead, the researchers predict that intergroup contact that is superficial is perceived as unimportant and will, therefore, not contribute to substantially diminishing ethnic or racial biases. Additionally, Van Dick et al. (2004) content that contact described as important will also be perceived as positive, and that those who perceive their intergroup contact experience as important hold less prejudiced attitudes than those who perceive their contact experience as unimportant. They explain that "important beliefs are more accessible and likely to be activated spontaneously, and strong attitudes based on these beliefs, in turn, are more stable over time, more likely to influence behavior, and less susceptible to persuasion and change" (Van Dick et al. 2004: 212). Their research suggests that, indeed, subjective reports of perceived importance of intergroup contact experiences mediate the positive outcome of reducing negative out-group attitudes. They further discover that perceived importance emerges when the contact is considered to contribute to a personal goal. Three reasons are offered to explain this link between perceived importance and more positive out-group attitudes during intergroup contact: (1) when individuals hold particular topics or experiences as important, they are motivated and involved in developing accurate perceptions of this topic; (2) when individuals perceive experiences and relevant topics as important, they are more likely to spend the cognitive effort 
required to form lasting attitudes, and; (3) when individuals perceive intergroup contact as important there is less chance the individuals' attitudes will be susceptible to outside information sources, such as the media and third-parties (Van Dick et al. 2004: 220-221). This suggests that understanding the perceptions the actors have about their intergroup contact experiences is mutually exclusive from identifying their out-group attitudes, and a critical factor in determining the likely outcome of contact.

\section{Additional Optimal Conditions in Light of Contemporary US Racism}

I now turn my attention towards the theories that explain the form of contemporary US racism reviewed above and in the previous section in order to propose additional optimal conditions for interracial contact that need to be added to theory that has evolved from Allport's work.

From Dovidio and colleagues we discover that racial attitudes take two forms: implicit and explicit. Implicit attitudes can, and often do, contradict explicit racial attitudes and are triggered during situations that are unfamiliar. It is difficult for whites to control the influence of implicit racial attitudes because they are not consciously available and affect unconscious behaviors, such as non-verbal communication (Dovidio et al. 2002). Their research underscores why miscommunication is an all too familiar outcome of interracial interactions and offers insight into specific conditions that could decrease the amount of influence implicit racial attitudes have during intergroup contact. When there is ample time to determine the consequences of one's actions, and the activity is important to the actor, explicit attitudes are reflected in their interactions. On the other hand, if there are time constraints that prevent adequate time to assess one's 
behaviors, and the task at hand is unimportant to the actor their implicit attitudes will dictate their behaviors during interaction (Dovidio et al. 2002).

Based on the research from Dovidio et al,. two additional conditions can and should be added to Allport's ICH: (1) the roles of each participant are clearly defined upon engaging in interracial contact, and (2) the in-group member is self-aware and cognizant that our behaviors are all subject to contradictions between our implicit and explicit attitudes, and that those we are interacting with often form impressions of our racial attitudes based off of the mixed messages that result from these contradictions. The first condition (explicitly designated roles) will help to reduce feelings of anxiety and uneasiness by providing a framework that elucidates the appropriate behaviors and actions during the contact situation (Dovidio et al. 2002b) This condition is distinct from Allport's condition of working toward a shared goal, although both conditions appear to complement one another. For example, the actors can have a clear task at hand while at the same time being unclear about each one's particular role in accomplishing the said task, which could potentially lead to anxiety or conflict. The second condition (selfawareness) might mitigate some of the influence of implicit racial by simply being cognizant of their existence and influence during intergroup contact. Understanding how implicit attitudes undermine positive communication can motive the in-group member, particularly if the individual's explicit attitudes are positive and egalitarian, to take the time needed to reduce the influence of implicit racial attitudes. Individuals who have a high level of self-awareness are less likely to be defensive and more likely to engage in self-critical (or self-reflection) thinking than those who do not exhibit self-awareness (Allport 1954). It follows then, that if this condition is present the in-group member will 
be more open to acknowledging and examining their unconscious biases; and therefore, will make the effort to work with their implicit attitudes instead of being hindered by them. It is plausible that there are individuals who are self-aware, but not in all social situations. This raises the question: Are there other contact conditions that encourage self-reflection? Other optimal factors identified by the ICH literature can, perhaps, supplement the practice of self-reflecting, such as the contact is frequent (Allport 1954), which provides ample opportunities to be self-critical; and, this frequent intergroup contact evolves into a relationship that is important to those involved (Van Dick et al. 2004), which often motivates people to self-reflect on how their partner's impressions are affected by both their own verbal and non-verbal communication cues.

Next, I turn towards Trepagnier's theory of “silent racism” to propose another addition to Allport's intergroup contact hypothesis. Trepagnier (2010) argues that most white Americans who identify as "not racist" actually do, often in subtle and unconscious ways, contribute to everyday racism and exhibit forms of silent racism. These forms of silent racism refer to white Americans' negative beliefs about black Americans and other people of color that result from the internalization of negative and false cultural information about African Americans (Trepagnier 2001). Ironically, it is the very notion of not being racist that prevents these individuals from examining their own racial attitudes and beliefs. For this group of white Americans (those who are "not racist"), it is not a conscious dislike or hostility contributing to their prejudices; rather, it is a lack of race awareness. Trepagnier (2010) explains that a high level of race awareness involves an awareness of how historical, societal, and personal contexts of racism interact with one another to advantage white Americans and limit the life chances of non-whites. Race 
awareness calls for self-reflection, but this is only one piece of developing race awareness; it also requires the ability for social-reflexivity, understanding the relationship between power and privilege. Thus, individuals with high levels of race awareness are less concerned with if they are racist and more concerned with how their behavior might be protecting white privilege.

From Trepagnier's discovery and description of silent racism, I suggest a new condition to add to the ICH: whites who exhibit high levels of race awareness prior to or during contact and/or interracial contact that enhances race awareness will increase the contact's ability to reduce racial prejudice. However, we are still left asking what, specifically, are the features of interracial contact that might promote race awareness? There is not a detailed blueprint for interracial contact that if followed correctly will heighten every white person's race awareness, as we are all constrained by our unique personality characteristics. Despite the subjectivity that must be considered, Trepagnier (2010) does suggest certain practices that are likely to foster the development of race awareness and mutual understanding during interracial interactions. For example, she finds that, among persons that demonstrate a high level of race awareness, all of them had one or more close relationships with people of color and engaged in conversations about race and racism with these individuals. Trepagnier (2010) explains that conversations about racism between whites and people of color can serve to disabuse whites of the often false racial information they use to understand racial issues (i.e., racial inequality, the impacts of discrimination, white privilege); thus increasing whites' race awareness. While on the surface this appears to put the responsibility on minorities to enlighten whites about contemporary racism, this is not the case. These discussions should be 
initiated by white individuals for two reasons: (1) it helps to ensure whites are interested in learning more about racial issues, and (2) it encourages whites to acknowledge that there are different understandings and perspectives.

I do not contend that cross-race relationships guarantee an increase in race awareness for the white individual. It is quite possible to be involved in interracial relationships that never address race and racism, particularly among those who disregard how race and ethnicity impact and shape our interactions (i.e., color-blindness). Moreover, if discussions about racial issues do transpire during interracial contact there is a risk that this topic will prove unproductive and create more distance between the individuals, particularly if many of the other optimal conditions are absent from the contact situation. Thus, reiterating Pettigrew and Tropp's (2006) argument I presume this condition would have the strongest effect, not in isolation, but in conjunction with additional optimal conditions, such as frequent contact, institutional support, equal-status within the contact, the relationship is important, mutual liking arises, and group saliency.

Finally, I turn towards Bonilla-Silva's work that intends to make sense of contemporary racism in order to develop new conditions for Allport's contact hypothesis. He argues that in the post-civil rights era a color-blind ideology has come to permeate whites' perceptions, as a group, on racial issues. Following a color-blind perspective, many white Americans use nonracial explanations to explain racial inequality (BonillaSilva 2003). This color-blind discourse serves to protect racial inequality (and thus, white privilege), and ultimately prevents color-blind whites from examining their own racial attitudes and developing empathy for minorities. Color-blind racism does not necessarily 
arise because of a conscious dislike for Americans of color; rather, it is an ideology, not an individual attribute, that is informally taught and learned throughout the socialization process (Bonilla-Silva 2003) and a result of continued racial segregation and white isolation.

Although a color-blind ideology permeates whites as a group, not every white American adheres to a color-blind way of thinking. Indeed, Bonilla-Silva (2003) discovers a small number of white participants who both acknowledged the pitfalls of color-blind thinking, and rejected it as a valid framework for understanding the racial status quo. He described this small sub-sample of whites as racial progressives who do not conform to "white pattern[s] of social interaction" (p. 180); they engage in interracial relationships, friendships, and intimacy.He identifies common factors among the white participants who are racially progressive: (1) working or lower-middle class backgrounds (2) female (3) close and meaningful relationships with non-whites (4) a liberal or politically radical outlook, and (5) attend racially integrated schools in diverse residential areas (Bonilla-Silva 2003:146). The above factors have little impact on whites' racial attitudes when found in isolation; it is when they are found in conjunction with one another that they are most likely to result in racially progressive attitudes among whites (Bonilla-Silva 2003).

Upon considering Bonilla-Silva's contribution to uncover the nature of contemporary racism, I propose the following additional conditions be added to the intergroup contact hypothesis: (1) for in-group members to bring with them prior intergroup experiences, and/or (2) the in-group member has experience with and 
knowledge of systems of oppression (shared lived experiences). First, it is reasonable to assume that every interracial interaction one participates in is a building block towards developing a broader understanding of how race and ethnicity impact our lives and social interactions. Still, prior experiences that are perceived as positive interracial interactions are more likely to increase intergroup contact's ability to reduce racial biases and negative attitudes than those considered negative. Bonilla-Silva (2003) emphasizes the significance of interracial interactions and relationships that involve equal-status between the actors. This supports the notion that early-life interracial interactions have the greatest impact on whites' later life preferences and choices (Braddock and Gonzalez 2010), as these are likely to involve equal-status relationships. The second condition speaks to Bonilla-Silva's finding that the small sample of racially progressive whites he examines are all members of out-groups themselves (i.e., gender, class). Oppression, prejudice, discrimination, and privilege are not solely linked to race and ethnicity; just as they all take various forms they are also a product of various human attributes and affiliations, such as religion, gender, class, sexual identity, physical capabilities, etc. If this condition is present there is the potential to develop mutual understanding and empathy through their shared experiences with group oppression. These conditions are not meant to suggest that whites who engage in interracial contact with a dearth of prior interracial experience are incapable of reaping the benefits of intergroup contact (or that those with past experiences are guaranteed to benefit from optimal interracial contact), or that every white person who identifies with another type of out-group is destined to develop a strong racial awareness because of optimal interracial contact $^{3}$. Rather, these are optimal

\footnotetext{
${ }^{3}$ There is a significant portion (even the majority) of white Americans who identify with one, or more,
} 
conditions that increase the potential for positive interracial contact to diminish racial biases and prejudice.

At this time, I layout a refined intergroup contact theory that incorporates

Allport's original premise, subsequent ICH research, and the additions illuminated by research on contemporary racism (see Table 1).

nonracial out-group but are still unable to fully understand, or empathize with victims of, racial oppression. This can be because they do not realize that there are varying degrees of oppression, or that oppression is compounded with every out-group one identifies with (multiple levels of oppression), which suggests that white privilege continues to protect whites from experiencing the same levels of oppression as their nonwhite counterparts in their same nonracial out-group. If a white woman claims all oppression is the same, she is ultimately invalidating and rendering invisible black women's experience of racial oppression compounded with gender oppression, and all other people of color who belong to multiple out-groups. 


\begin{tabular}{|c|c|}
\hline \multicolumn{2}{|c|}{$\begin{array}{c}\text { TABLE } 1 \\
\text { INTERGROUP CONTACT THEORY: A MODEL FOR INTERRACIAL CONTACT }\end{array}$} \\
\hline $\begin{array}{ll}\text { Optimal Condition } \\
\end{array}$ & Source \\
\hline 1. Frequent contact & Allport \\
\hline 2. Institutional support & Allport \\
\hline $\begin{array}{l}\text { 3. Support from above ensures equal-status within } \\
\text { contact }\end{array}$ & Allport \\
\hline 4. Cooperation for a shared goal & Allport \\
\hline 5. Mutual liking & Pettigrew and Tropp \\
\hline 6. Group saliency & $\begin{array}{c}\text { Pettigrew and Tropp; Van } \\
\text { Oudenhoven et al. }\end{array}$ \\
\hline 7. Contact is important to all involved & Van Dick et al. \\
\hline 8. Clearly defined roles & Dovidio et al. \\
\hline $\begin{array}{l}\text { 9. Aware of the potential impacts of implicit racial } \\
\text { attitudes in interracial interactions }\end{array}$ & Dovidio et al. \\
\hline 10. Race awareness develops or is enhanced & Trepagnier \\
\hline $\begin{array}{l}\text { 11. White individual has formed meaningful relationships } \\
\text { with nonwhites before }\end{array}$ & Bonilla-Silva \\
\hline $\begin{array}{l}\text { 12. White individual is part of a nonracial out-group that } \\
\text { experiences oppression }\end{array}$ & Bonilla-Silva \\
\hline
\end{tabular}

This new model for the contact theory appears to be an effective road map for broadening our understanding of cross-race mentorships. Still, there are questions that emerge based on this model of the theory. For example, how are these optimal conditions established? Are some more important than others? Can intergroup contact that does not meet these conditions still engender the positive result of reducing prejudice and biases? 
These are important questions and this research aims to make contributions to these questions, among others. What I am purposing at this time is that these conditions are not standalone conditions; they work together as a package. Just as Pettigrew and Tropp (2006) confirmed that Allport's conditions do not significantly impact intergroup contact's outcome on their own, but when present together. Thus, I understand these conditions as an aggregate; it is how they reinforce and interact with one another that is the driving force behind structuring intergroup contact capable of diminishing racial biases and prejudice. These conditions are part of a process where intergroup contact is the catalyst and a reduction in out-group prejudice is the outcome. In other words, these optimal conditions are the mechanisms for this desired result.

\section{Hypotheses}

1.) Protégés and mentors who perceive their cross-race mentoring relationship as very successful will be those who experienced all or most of the conditions in Table 1.

2.) Protégés and mentors who perceive their cross-race mentoring relationship as somewhat successful will be those who experienced some, but not most, of the conditions in Table 1.

3.) Protégés and mentors who perceive their cross-race mentoring relationship as not successful will be those who experienced none or very few of the conditions in Table 1. 


\section{CHAPTER IV: RESEARCH DESIGN}

\section{Purpose and Significance}

This study explores a special type of cross-race relationship; one that, in addition to providing adult mentors for minority youth and young adults who need them, potentially serves to improve relations between groups. This study seeks to increase knowledge in both the areas of mentoring and interracial interaction theories; specifically, the contact hypothesis, as it has not yet been explored within the context of cross-race mentorships. Furthermore, by identifying obstacles that prevent, as well as practices that facilitate integration in transracial mentorships, this research can provide insight on how to structure successful cross-race mentoring interventions.

The purpose of this in-depth interview study is to explore thementoring perceptions of adult and student participants involved in cross-race mentorshipswithin higher education. These data are collected from onementoring program, New Horizons, in a local university, University of the Pacific Northwest (UPN). For this specific research, adult and student participants in a cross-race mentoring relationship is understood to include white adult mentors and African-American or Latino student protégés who are first generation college students soon to make the transition from a local community college (Northwest Community College) to University of the Pacific Northwest. Additionally, mentoring perceptionsrefer to the participants' personal understandings in regards to their activities, interactions, and descriptions of their mentoring experience.

The specific research questions I seek to exploreare: 
1.) How does each participant describe their interracial mentorship?

2.) How do the participants' expectations and assumptions shape their mentoring experience?

The last several decades have seen a rise in qualitative inquiries (Marshall and Rossman 2011); particularly, in studies examining racial attitudes and understandings and the process of developing empathy (Bonilla-Silva 2003; Feagin 2001; Trepagnier 2010). Moreover, quantitative methods run the risk of suppressing valuable data byprescribing a narrow worldview for the participants through predetermined operational variables (Marshall and Rossman 2011). On the other hand, qualitative methods underscore the value of letting participants articulate their lived experience with their own words and subjective understandings. This specific research is not concerned with traditional and quantifiable mentoring outcomes such as increasing academic motivation, enhancing academic and career aspirations, or protégé behavioraloutcomes, but rather, it seeks to understand how protégés of color and white mentors understand and reconcile the racial dynamics that emerge within this dyadic relationship. This remains an understudied aspect of mentorships (Hansman 2001; Rhodes et al. 2002; Spencer 2006).

Given this study's focus on the participants' meanings and perceptions of their interracial mentoring relationship, a qualitative approach is the most suitable choice for generating these data. An in-depth interview design is appropriate because the best way to understand participants' lived experiencesare through detailed descriptions of their experiences in their own words (Marshall and Rossman 2011). 


\section{Site and Participant Selection}

With the support and efforts of the mentoring program's (New Horizons) staff, cross-race mentoring pairs were selected for one-on-one semi-structured interviews. The focus and nature of the research questions demands a purpossive selection method. The criteria for subject recruitment entails that the mentor is a white adult and the mentee an African-American or Latino student. In utilizing the staff to select potential participants, we aim to eliminate as much of the researchers' biases as possible. This particular sitewas selected because of the prevalence of mentoring interventions within education andaccess to the sample.

These data come from a formal mentoring program, which is designed to assist first generation college students expecting to make the transition from NorthwestCommunity College (NCC) into a four-yearstate university, University of the Pacific Northwest. This mentoring program focuses on first-generation students from underrepresented groupswithin higher education, and aims to increase retention rates and establish a support system for students experiencing a paucity of resource endowments. For this reason, applicants are selected and purposively matched with specific faculty members at PacificUniversity. The protégés spend the summer assisting a faculty member with their current research project(s). The hope is that the protégés experience a supportive mentoring relationship and gain a clearer understanding of the university's culture and expectations in regards to student course load and work. While the program does not have this explicit aim, in practice, this program intends to cross cultural, racial, and class boundaries in a manner that encourages the development of a supportive and enduring mentorship. This site was selected because of the researchers' personal 
connection with the program developers and the program's emphasis on mentoring students from underrepresented groups. Thus, this program ensured that a considerable amount of interracial mentorships would be available to sample from.

Given that the focus of this research is to gain a better understanding of how race and ethnicity structure interracial mentorships, the researcher, guided by the current sociological study of race relations and mentoring research, selected protégés who identify as either African American or Latino. Much of the literature concerned with racial attitudes and biases center on the Black-White divide in the United States (Bobo 2001; Bonilla-Silva 2003; Dovidio et al. 2002; Feagin 2001; Trepagnier 2010), while Hispanic Americans' and Latino immigrants' experiences with discrimination has increasingly become a major area of inquiry within the social sciences (Berends and Peñaloza 2010; Goldsmith 2004; Massey 2004) and the mentoring field (Diversi and Mecham 2005; Riegle-Crumb and Callahan 2009; Santos and Reigadas 2002).

\section{Sampling Frame}

These data consisted of eleven protégés, who identify as either black or Latino, from cross-race mentorships involving a white faculty member. The racial/ethnic and gender breakdown for the protégés include four black male protégés (one of which identifies as bi-racial,black and white),two black female protégés, two Latino male protégés, and three Latina female protégés.From this sample, one Latina female and one black male are foreign-born. Three of the protégés participated in the formal mentoring program during the summer of 2010 and eight participated in the program during the summer of 2011. The data from protégés were collected durig the winter of 2012. 
A number of meetings were held between the two lead researchers and the program developers to provide an overview of the study, address any concerns or questions the program developers had, and create a sampling plan that ensured the protection of the protégés' identities from the researchersuntil the students agreed to participate in the study. The program leaders generated a list of the mentoring pairs, which included the name of the faculty mentor and provided a random number in place of the protégé's name, alongside their gender and racial/ethnic identification. The list included thirty-three mentoring pairs; however, some mentors had more than one protégé, so there were more student protégés than faculty members.

From the list, the researchers determined that ten pairsdid not constitute an interracial pair. From the remaining list, two additional pairs were eliminated from the sample because, althoughinterracial pairs, the student mentees identified as Native American and the decision was to limit the scope of this study to black and Latino mentees. With a sampling frame of twenty potential protégé participants, an invitation letter ${ }^{4}$ was drafted by the researchers and subsequently edited, signed, and sent to the homes of the students by the program coordinators. The letters were then followed up with a phone call from a former protégé in the program who was not included in the sampling frame. This student was asked to conduct the follow-up phone calls for two reasons: 1.) because she was part of the mentoring program she was familiar with the other mentees and this still protected the students' identities from the researchers; and 2.)

\footnotetext{
${ }^{4}$ The invitation letter was written from the perspective of the program coordinators. It described the nature of the research, invited the student to participate in one interview, and explained that following the interview the student would receive a cash incentive for participating. See appendix A to read the actual letter.
} 
the researchers and program leaders believed that because she was also a student, less pressure or coercion would be felt by the potential participants to agree to take part in the study. However, because more than a year had passed for some of the students since their involvement in the program, some addresses and numbers were no longer current. Out of the twenty students in the sampling frame, six were unable to be reached. It is unclear if those students received the invitation letter and avoided the follow-up phone calls or if their address and phone number wasno longer current. Fourteen potential participants were contacted by phone and thirteen agreed to participate, only one student explicitly declined to take part. However, two of the thirteen who agreed never found a time to schedule the interview.

There were several faculty mentors involved in multiple interracial mentorships; therefore, the sampling frame of mentors consisted of thirteen faculty members. The mentors were not contacted until after the interviews with the protégés. The researchers agreed that contacting the students first would ensure less pressure on the students to agree to participate. In this sense, it prevented the scenario of a mentor telling their student protégé about the study and subsequently applying pressure on the student to participate, whether intentional or unintentional on the mentor's part. Additionally, we did not invite the protégés and mentors concurrently to increase the likelihood that the participants would not know if their mentoring counterpart participated, as this could also increase pressure to take part. Hence, regardless of which student mentees agreed to participate, all mentors involved in interracial mentorships were contacted and invited to participate in the research. From the sampling frame of thirteen mentors, nine agreed to participate. One mentor was unavailable to participate and three never responded to the 
two emails sent to them. Of the nine mentor participants, six are female and three are male. Eight of the mentors are white, and one was not; however, the minority mentor was involved in an intercultural mentorship with a minority student who is ethnically and culturally from a different background.

\section{Interviews}

The interviews were semi-structured, allowing each participant to use their own personal style to convey their narrative. Each interview lasted between 45 - 70 minutes. Marshall and Rossman (2011) suggest in-depth interviewing as an effective method for better understanding an individual's lived experience. This study is designed under the assumption that in order to grasp the true personal meaning of an experience, the participants' own words are required. Before the interviews begin, the interviewer obtained written consent, described the present study, and addressed any questions or concerns the participants had. It was imperative to obtain written consent from the student protégés and faculty mentors, and we made every effort to gain their trust and address any concerns. We were sensitive to the students' psychological well-being throughout the interview. Names were changed and identities were protected from the beginning to ensure confidentiality (Marshall and Rossman 2011).

The questions were open-ended and followed up with probing questions to incite further elaboration and clarification. The interview explored each protégé and mentor's experience and perception of their mentorship and of one another. Many of the questions asked the participants to reflect on the type of connection they formed with their mentor 
or mentee, how racial or ethnic identity influenced this relationship, and how the structure of the program contributed to the mentorship.

With the hope of eliciting more candid responses, interviews were raciallymatched (Rhodes et al. 2002; Spencer 2006). One of the researchers, a white woman, interviewed the white adult mentors; two additional graduate students, a black male and a Latina female, and one advanced undergraduate student, a black female, interviewed the minority mentees. When possible the interviews were gender-matched as well ${ }^{5}$. The investigatorsare aware of the potential risks in race-matched interviewing: of assuming a false sense of implicit understanding (Marshall and Rossman 2011) and made a deliberate effort to avoid any such assumptions (Spencer 2006). In order to avoid such pitfalls, the interviewers were trained to engage in probing questions even when they felt like they related to what the participants said. Meetings were held with the interviewers to practice with the interview guide, refine the instrument, and discuss the interviewing process. Following the interviews, I met with each interviewer to reflect and discuss. These meetings proved helpful in discovering how interviewers can assume to understand the participants' response and forgo probing questions. Prior to meeting with the interviewers, I listened to the interview and was able to offer specific examples of when these instances occurred. By identifying these instances, the interviewers became more aware and were then more effective at preventing an assumed understanding.

\footnotetext{
${ }^{5}$ Two of the Latino male protégé interviews were not gender matched; three of the male mentor interviews were not gender matched. The rest of the sixteen interviews were gender matched.
} 


\section{Data Analysis}

The interviews were transcribed as they were completed; thus, analysis and data collection occurred in concert to better inform one another. This not only assisted in timemanagement, but also allowed for the refinement of the data collection instrument: the interview guide. Upon listening to the first couple of interviews, the researchers and interviewers were able to identify certain questions and probes that were eliciting rich data and those that needed additional probes. We also found that reordering some of the questions was necessary. For example, following the first few mentor interviews, we discovered that moving questions explicitly about racial and ethnic differences to the end of the interview allowed for a rapport to develop before engaging in questions specifically centered on race and ethnicity. One of the strengths of conducting qualitative research is its inherent flexibility (Charmez 2006), which encourages the researcher to acknowledge her own biases and take proactive steps to mitigate the influence of these biases or previously held assumptions. Once an interview was transcribed it was then listened to again in conjunction with reading the transcript to ensure accuracy.

\section{Coding of the Cross-Race Mentoring Outcome}

The next phase of data analysis involved the listening guide method. This strategy requires no less than three detailed readings (listenings) per transcript (Gilligan et al. 2003) in order to stimulate the discovery of nuances in these data. Each listening focused on a few key factors: the main point of the stories being told, how participants represented themselves, and the meanings they attached to their mentoring relationships (Spencer 2006). It was during this listening phase that each interview was coded as (1) 
successful, (2) medium success, or (3) low-to-no success. In light of a refined model for the intergroup contact theory and the hypotheses I presented in the previous chapter, it was important to discern the level of success the participants perceived in respect to their mentoring experience and relationship.

If the participant described their mentoring experience as positive, the relationship as one involving a close interpersonal bond, there was a high level of agreement between the mentoring partners, and both mentor and protégé identified personal and/or professional gains or growth the interview was coded as a success.

Mentorships coded with a medium success outcome were those described as somewhat positive, but not as a negative experience; lacked a close bond between the two, and only discussed their mentoring relationship as a professional one; there was not a high level of agreement between what the mentor discussed and the protégé described; and the mentor and/or protégé did not explain how their relationship moved their goals forward.

Finally, low-to-no success mentorships where coded as such when the participant explicitly described their experience and/or partner in a negative manner; there was not a relationship that developed between the mentor and protégé; the mentor and/or the protégé did not discuss anything beyond the research project that the pair engaged in; and the protégé was not engaged in the research tasks.

\section{Coding of the Conditions Present in the Cross-Race Mentorship}


Because I am interested in the perceptions and understandings of both members of a cross-race mentorship, the unit of analysis is a complete mentoring pair (mentor and protégé). Therefore, the next phase of analysis concentrated only on the participants whose partner (either protégé or mentor) also participated in an interview. From the eleven protégé participants, six included a mentor interview; from the nine mentor participants, seven included a protégé interview (one pair consisted of two mentors and a protégé). In other words, there are six mentoring pairs, with one 'pair' actually involving three individuals. The interviews from each pair were then compared to one another to confirm if their mentoring relationship was a success or less than a success. Thus, successful mentorships were perceived as such by both participants. Following this, each interview was coded for the optimal conditions (see Table 1). The transcripts were coded if the condition was present and if the condition was absent. Not every single condition could be determined as present or absent in every interview, as some participants answered the questions without clearly identifying the condition or with ambiguous explanations that did not include sufficient information about that condition. Below each condition is listed with my understanding of what constitutes that condition.

Frequent Contact: If both participants indicate agreement about meeting face-to-face on a weekly basis (at least once a week) throughout the duration of the mentorship the pair is coded as involving frequent contact. If both participants describe their face-to-face contact as less frequent than weekly, or that the work consisted of primarily independent work the pair is coded as not involving frequent contact. If there is a lack of agreement between the two, the pair is coded as not involving frequent contact. 
Institutional Support: If both participants describe the structure of the mentoring program as instrumental in creating a positive mentoring experience the pair is coded as having institutional support. This means that the mentoring program's staff provided ongoing support throughout the mentorship (DuBois et al. 2002), not just at the beginning, and both protégé and mentor clearly understood the goals of the program. If there is not agreement from both participants about adequate support, training, and/or criteria for matching the pair the pair is coded as not involving institutional support. If either participant describes the program's structure in a negative way the pair is coded as not involving institutional support.

Equal-Status: The condition of equal-status is a difficult condition to establish in a mentoring relationship comprised of faculty members at a university and first-generation community college students. However, I determined that equal-status could arise in a number of areas in this type of relationship. For example, when both participants describe the mentoring relationship in terms of an employer-employee dynamic I consider this to not indicate equal-status (Allport 1954); however, if the participants explain their relationship as involving teamwork and a collaborative dynamic I determine the presence of equal-status is established. This is further supported when the two describe a friendship and not a superordinate/subordinate relationship. When the participants confirm that both mentor and protégé's goals were equally considered in the relationship I determine the presence of equal-status. Additionally, equal-status is coded as present when the two convey a process of mutual exchange occurring between the pair.For example, both the mentor and protégé indicate they learned and grew from the relationship. However, I considered equal-status to be absent when: the mentors' research 
agenda was the primary, or only, focus of the research tasks; the protégé was only allocated mundane research tasks that are not typically done by a lead researcher (i.e., when the protégé was not able to step into the role of the lead researcher); the mentor did not make an effort to learn about or advance the protégé's academic and career goals; and the relationship was described as solely a professional relationship where the protégé was working for the mentor.

Cooperation Toward a Shared Goal: If the participants describe their relationship as contributing to a personal goal, because of activities or tasks they both participated in, the pair is coded as cooperating towards a shared goal. If the participants report particular issues or problems they worked out together the pair is coded with this condition as present. If the participants explain the mentorship as only benefiting one of the participants, the pair is coded as not involving cooperation. If the participants convey a sense of competition or there is a lack of agreement about cooperation, the pair is coded with cooperation as absent.

Mutual Liking: If both participants talk about one another with positive descriptions and report instances of personal sharing between the two that brought them closer together the pair is coded as involving mutual liking. If either participant describes their partner in a negative way or indicates that there were negative interactions between the two, the pair is coded with the condition of mutual liking as absent.

Group Saliency: If both participants mention their mentoring partner as belonging to their respective racial or cultural groups on more than one occasion, the pair is coded with the condition of group saliency. If the participants are unaware of their partner's 
ethnic or racial background, the pair is coded as not involving group saliency. If either participant explains that they never thought about or considered their mentoring partner's ethnic background, the pair is coded without the presence of group saliency.

Perceived Importance: If both participants describe their mentoring experience as meaningful and instrumental in advancing personal goals, the pair is coded as having perceived importance. If one or both participants explain their mentoring experience as simply a vehicle for advancing the mentor's research agenda and/or as unrelated to the protégé's academic/career goals, the pair is coded as lacking perceived importance. If the mentor reports their motivations and goals for becoming a mentor as contributing to a more inclusive and welcoming university, for first-generation students and the protégé reports developing important academic skills and expanding their social network at the university the contact is coded as involving importance.

Clearly Defined Roles: If both participants indicate a clear understanding of one another's and their own expectations and goals for the mentorship, research project, and how each other will contribute to these the pair is coded as having the condition of clearly defined roles. If either participant is unclear of what the program or each other expect from one another, the pair is coded as not involving the condition of defined roles. If the participants describe clear and consistent communication among one another, the pair is coded with this condition present.

Aware of Implicit Racial Attitudes: If the participants acknowledge the prevalence of racial stereotypes during interracial interactions, the pair is coded as involving the condition of aware of implicit racial attitudes. If the mentor claims that racial or ethnic 
affiliation had no effect on their interracial mentoring relationship or that they never thought about their protégé's ethnic background, the pair is coded as not having this condition.

Race Awareness: If both of the participants mention racial barriers and/or race privilege as significantly impacting our life outcomes the pair, is coded with the condition of race awareness as present. If the participants claim that racial differences do not matter or that they never thought about how their racial and ethnic backgrounds might affect this experience or relationship, the pair is coded with race awareness as absent.

Mentor has Developed Meaningful Interracial Relationships Before: If the mentor discusses building meaningful intercultural relationships prior to this mentoring experience and how the past relationships influence the current one, the mentor is coded with this condition present. If the mentor reports that this is their first interracial relationship, the mentor is coded as lacking this condition. This condition is anchored in the work of Bonilla-Silva (2003) and is related to how whites might develop empathy and is therefore directed at the white individual who is involved in interracial contact.

White Mentor is a Member of a Nonracial Out-Group: If the white mentor discusses experiences of prejudice or discrimination because of their own background or identity as a way to relate or find common ground with their protégé of color this condition is coded as present. The mentor may discuss this with their protégé or they may just mention it in the interview while reflecting on their mentoring experience. If the mentor does not mention experiences or membership in a nonracial out-group, this condition is coded as absent. 
Once the mentoring pairs were identified as either successful or less than successful and the initial coding was complete, the pairs were grouped together by their success outcomes and coded again to verify and refine the codes. During this phase I began to parse out the quotes that illustrated the conditions in the participants' own words. With these quotes I was able to create tables that provided a visual for how often the different conditions were discussed and in what forms the conditions took.

Additionally, I created a map with the coded data, which ultimately helped clarify and conceptually demonstrate the conditions' interconnectedness (Gary 2009).

Finally, the additional interviews (fiveprotégés and two mentors) were coded and are described in the findings chapter to supplement the mentoring pairs' data. While these latter interviews could not provide me with the mentoring partner's perspective on the relationship, this data was used to confirm the results of the mentoring pairs or discover new patterns among the conditions. Additionally, this helped to establish if saturation had been reached or if more data was needed. 


\section{CHAPTER V: FINDINGS}

A variety of mentoring experiences were described by the mentors and protégés, which I then categorized as high success, medium success, or low-to-no success crossrace mentoring experience. Two of the nine mentors and five of the eleven protégé participants that were interviewed did not have a mentoring partner that participated in this study. Thus, there were six complete interracial mentoring relationships (one pair is actually a trio, as one protégé had two mentors) that generated the bulk of these data.The remaining seven participants were still analyzed and were used as supplemental data to support and refine the findings from the six complete pairs. Of the complete pairs, two were labeled with a successful outcome, two with a medium success outcome, and two with a low-to-no success outcome. The following discussion is organized according to their determined outcome, with each outcome related to one of the three hypotheses. Following that is a discussion about the structure of the program.

Throughout this chapter I reference a poster presentation, which was a requirement that the mentoring program, New Horizons, set up for the protégés. The poster presentation was to be designed and prepared by the protégés to delineate the research project they worked on with their mentors. At the end of the formal mentorship New Horizons held a conference modeled on professional scientific and academic conferences where the protégés presented their posters; their mentors, family, and friends were encouraged to attend. For some mentoring pairs this was an activity that facilitated collaboration between mentors and protégés, and will be discussed throughout this chapter. 


\section{Successful Cross-Race Mentoring Experiences}

This section delineates the successful cross-race mentoring experiences by examining the participants' descriptions of their mentoring partner and relationship, and identifies which conditions are present. Thus, this section addresses Hypothesis \#1: protégés and mentors who describe their cross-race mentoring relationship as very successful will be those that involve all or most of the conditions in Table 1. Three subsections organize the following discussion on successful interracial mentoring experiences: (1) Expectations and Perceptions (2) The Mentoring Relationship and (3) The Racial Component. There are two complete mentoring pairs that are considered as successful interracial mentoring experiences. Pair \#1 includes a white female mentor (Karen)and a black male protégé (Ryan). Pair \#6 actually consists of two white mentors ${ }^{6}$, one female(Julie) and one male(Tom), and a Latina female protégé (Maria).The supplemental data derives from three protégé interviews, which include one black female (Candice), one black male (Paul) who is also an international student and has only lived in the United States for a few years, and one Latino male (Alex). In addition to the protégés there are also two mentors who described successful intercultural mentoring relationships. The two mentors involve one white female (Laura) and one male (Eddie) who is the only mentor that does not identify as white ${ }^{7}$. The five participants in this supplemental data group are considered such because their mentoring partner did not participate in this research; therefore, it cannot be confirmed that both members of the

${ }^{6}$ Although there are actually three participants in Pair \#6, I refer to them as a pair throughout this section for the sake of consistency. A slightly different interview guide was created for Julie's interview, as she did not perform the lead mentor role, but still spent a significant amount of time with Maria, the protégé.

${ }^{7}$ The mentoring program had very few nonwhite mentors; therefore to protect Eddie's identity and ensure confidentiality I do not disclose his ethnic background. Even though Eddie does not identify as white he was still a member of an interracial mentoring relationship and his perceptions and understandings make a valuable contribution to this research. 
pair perceive the relationship and experience as a success. Despite this, the supplemental data do provide an opportunity to compare the findings from the complete pairs and search for similar or different patterns. To recap, the two complete pairs are Karen/mentor and Ryan/protégé (Pair \#1) and Tom/mentor, Julie/mentor, and Maria/protégé (Pair \#6); and the supplemental data group includes three protégés (Candice, Paul, and Alex) and two mentors (Laura and Eddie).

\section{Expectations and Perceptions}

This section describes the mentors' motivations and expectations that influenced their decision to be a mentor in this program, how the mentors and protégés describe one another, and how each member of the mentorship understands their relationship. Throughout this discussion I will identify how these descriptions relate to the conditions in Table 1. For example, the conditions that are associated with the mentors' motivations and expectations are perceived importance and cooperation towards a shared goal.

The mentors from this sample who are involved in successful interracial mentoring relationships (Karen, Tom, Julie, Eddie, and Laura) all shared a common motivation when choosing to take on the mentoring role. For these mentors the decision to participate in the mentoring program was centered on providing the student protégés with opportunities that could advance the students' educational and career aspirations. In fact, Karen and Laura were the only mentors in this group that even mentioned their own research when explaining why they were motivated to mentor. When Karen talks about her decision to become a mentor she also describes this role as fulfilling and that it makes her job more meaningful. Karen explains her motivations. 
Well gosh there are a number of reasons; one is that we did have this hole that was left by not having a master's student with us. Second thing is that part of our goal (as a department) is to get people into doing this work, and it's part of our goal to provide mentorship opportunities for people that are from underrepresented groups...And, then the other thing is it's just a lot of fun to share that with young people and I enjoy that part, being able to do teaching. I have had too many administrative responsibilities over the years and so it's really nice to have the one-on-one extensive contact with people over time.

Thus, all of the mentors indicated that they perceived this relationship as important and beneficial for both the mentor and protégé.

Three of these mentors, Karen, Tom, and Eddie, also highlightedthe importantce of mentoring opportunities for students from underrepresented groups within higher education. Eddie also highlighted how important mentoring is in the broader context of a healthy society when he explained why he decided to be a mentor. Eddie adds:

I didn't get here by myself; I got help and it doesn't matter how good you are, eventually you will need somebody to lend you a hand...I also look at the big picture: sometimes we do something special for an individual and in reality we're not doing it for that individual, we're doing it for the people that that individual represents. It goes actually in the university setting the same...If you step back and you realize, society benefits by having a lot of talent and society benefits by having people who are well educated...you're improving everybody's quality of life. So from that perspective I find it rewarding. I find it professionally challenging, yet intriguing to be able to mentor students who are not equally represented in high paying jobs as they are in a population.

These three mentors, Karen, Tom, and Eddie, all demonstrated perceived importance and expressed race awareness with their emphasis on underrepresented and minority students. These mentors' expectations and goals sets them apart from the larger sample; for example, when asked what their goals were for their mentorship and if they were met, 
these mentors mentioned the protégé's growth and progression in pursuit of their academic goals. The mentors' own research agenda played a secondary role in the mentoring relationship and instead, the protégés' academic development and self-efficacy were the focus of the mentorship. Laura illustrates this.

Yeah my experience met those expectations and beyond. You know, I mean seeing...there's one thing when somebody...I mean students always come to me and say, "I want to go to grad school," but to actually work with an undergrad at community college who says, you know, who is facing a lot of challenges and then to see she's really still thriving and headed in that direction and moving closer to that...yeah, it's very gratifying.

Thus, all of the mentors in the successful outcome group, Karen, Julie, Tom, Eddie and Laura, demonstrated perceived importance and cooperation toward a shared goal.

Among both the complete pairs in the successful mentoring group, all five participants (Karen, Ryan, Tom, Julie, Maria) described their mentoring partner and experience as extremely positive. Pair \#1, Karen and Ryan, even indicated that the condition of cooperation and equal-status within the contact were present in the ways they described one another. Karen began her description of Ryan by delineating what his academic and career goals were; while Ryan began his description of Karen by explaining how helpful she was. Ryan said:

Karen, she was as a person very nice. I mean very helpful. She really helped me out with a lot of stuff. She was very nice to me...showed me a lot of stuff. She brought me into a lot of the interviews. I got to even sit in on their staff quarterly meetings and stuff like that. So, I was very thankful for the opportunity.

Both mentoring pairs were labeled as establishing mutual liking. Pair \#1 (Karen and Ryan) was also coded with cooperation toward a shared goal and equal-status within the 
contact. Additionally, all the participants in the supplemental data group (Eddie, Laura, Paul, Alex, and Candice) were also categorized as having mutual liking.However, because these participants' mentoring partners did not participate in the study, it cannot be confirmed that the liking was mutual. Still, it was evident from Laura (mentor) and Alex (protégé) that they had developed a personal bond with their mentoring partners as their discussions revealed a significant amount of personal sharing amongst the mentoring pairs.

When the protégés were asked about their first impressions of their mentors four of the five protégés (Ryan, Maria, Alex, and Candice) in the successful group reported feeling nervous and self-conscious during the first meeting. Ryan, Alex, and Candice also noted that the racial, class, and educational differences created tense and uneasy feelings for them (personally) in the beginning of the mentoring relationship. Candice explains that:

Yes, definitely I felt self-conscious. Definitely not from her giving me that but just because I don't have anyone in my family that I was really raised by who was in higher education. So, that was just intimidating. I definitely feel we're different. I definitely have overcome a lot of issues from my upbringing and different things like that that make me feel apart from others who maybe...I grew up definitely not in, I was lower class. Yeah.

Ryan adds:

The first few meetings I was just like, "Wow!" Just the dynamics, you know, just an older white woman. We have our perceptions about older white women and how they see black men, so it probably played on me more than it played on them. So, it was just like all this, you know, these emotions and kind of just like, okay, wow. I'm in this position. I got to step up; I got to show that I'm worthy; I got to show them that I'm here to learn from all their knowledge. 
While Maria also noted feeling self-conscious when first meeting her mentors, she did not believe it was because of racial or cultural differences. Maria explained that, "Julie's personality is just so outgoing; whereas, I'm just more closed kind of me by myself and she's totally opposite." Similar to Maria, Paul also did not see how race and ethnic differences played any role during his first meeting with his mentor. Thus, three of the protégés (Ryan, Alex, and Candice) exhibited an awareness of how racial or cultural differences might engender stereotypes, particularly during the first few interactions with their mentor.

To sum up, the five mentors (Karen, Julie, Tom, Eddie, and Laura) were all labeled as perceiving their mentoring experience as important and all expected to cooperate with their protégé to further the protégés' academic and career goals. Both of the complete mentoring pairs in this successful group were also categorized as having mutual liking for one another. The participants in the supplemental data group also were coded as mutual liking being present, although this cannot be confirmed by their mentoring partner. Ryan (protégé) expressed being aware of implicit racial attitudes and explained how this made him feel self-conscious in the beginning of the relationship, while two other protégés (Alex and Candice) also mentioned the differences between themselves and their mentors in terms of race, education, and class.

\section{The Mentoring Relationship}

This section provides an overview on how much time the mentors and protégés spent together, how the respondents describe the activities and work the two engaged in, and how the protégés perceive this experience as contributing to their academic and 
career goals. This discussion will confirm if the above conditions were established within the mentoring relationship and indicate other conditions that are or are not present.

In order to determine how much face-to-face contact the mentoring pairs actually had with one another the participants were asked about their typical routine during the mentorship and specifically how much time the two spent with each other. The two complete pairs in this group, which are Karen and Ryan (Pair \#1) and Tom, Julie, and Maria (Pair \#6) all indicated that they had frequent contact with one another. Karen and Ryan met with each other at least once or twice a week during the mentorship, and are therefore categorized as having frequent contact. Julie and Maria worked together two to three days a week with each other and Maria met with Tom on weekly bases, so they too are considered to have the condition of frequent contact. While Laura and her protégé did not meet on a regular basis the two have since continued their mentoring relationship for almost two years are meet with one another bi-weekly, and are therefore considered to have frequent contact. Paul indicated that he worked with his mentor three to four days a week and is coded as having frequent contact. However, Candice was not clear in her interview if her relationship involved frequent face-to-face contact, so it cannot be determined if frequent contact was present in her relationship. Both Eddie (mentor) and Alex (protégé) indicated that they did not have frequent contact with their mentoring partner, at least not frequent face-to-face contact, so they are labeled as not establishing the condition of frequent contact.

The protégés were asked to describe the work they did with their mentor and what they enjoyed the most from their mentoring experience. Their responses indicated how 
engaged the protégés were in the work they were doing and shed light onto how important their mentoring relationship was to them. The presence of equal-status within the relationship emerges in some responses as well. All of the protégés in this group (Ryan, Maria, Alex, Paul, and Candice) described their mentoring experience as an opportunity to expand their social network both within the university and the larger community. Additionally, four out the five protégés (Ryan, Alex, Paul, and Candice) explained that their relationship provided them with opportunities to gain a clearer perspective on their academic goals. While Maria did not mention if the experience contributed to her academic or career goals she did indicate that the experience was important to her and that it contributed to her personal development. Candice reported that her mentor taught her more about "the steps to take to get into the graduate program" and that her mentor was "very open about how she can help me network with people to get me where I need to go." For Candice this inside information she received seemed to enhance her mentoring experience and the importance she attached to the relationship. Ryan explained that because his mentor, Karen, made sure to provide opportunities outside of the research experience he never felt like the relationship was just about the work he was doing for her.

Karen had me talk to different people so I could get a more full perspective of, like, what I want to do. So, I felt like that was a really good thing. I never felt at any point throughout the whole process it was just about getting my work done for her. I felt like she wanted me to learn something about what I want to do too.

Paul described his mentor as instrumental in providing him with the tools and support he needed to advance his writing skills in research. Before this mentoring experience, Paul did not have clear understanding of the difference between writing a good paper that tells 
a story and one grounded in empirical research. Paul explains that because of their collaboration he is now a much better and experienced researcher.

I discovered that my writing was not research based. So, then I discovered that even though I could write good papers, say about telling a story that is different than an argumentative essay. So, I discovered I'm capable of writing some papers, and if I wanted to learn how to write other papers then I have to also switch and then...in other words, she showed me where my strong points were and where my weak side was. So, then I discovered my strengths and my weaknesses.

All of the protégés indicated that their mentoring relationship contributed to their personal growth in one way or another, their mentor was an important piece of this personal growth. Therefore all of the protégés are labeled as describing a relationship with perceived importance and with the condition of cooperation.

Karen, Tom, and Julie confirm the conditions of perceived importance and cooperation towards a shared goal in their explanations of their mentoring relationship. In addition to this, the mentors indicate that there was equal-status within their relationship in respect to whose goals where being considered and how much input the protégé had on their research tasks. Karen, Tom, and Julie actually created research projects for their protégés, Ryan and Maria, to specifically align with their academic goals. Julie explained that it was important to her and Tom to get input from Maria about what interested her most before deciding what projects Maria would contribute to.

We wanted to make sure we were giving her stuff to do that was at least interesting because we had a lot of different things we could have had her work on, and so Maria helped decide in some ways what would help further her career goals. 
Similarly, Karen explained how she worked with Ryan to find a research project that fit with his goals and aspirations.

I decided to interview Ryan and he was just so impressive as a person that I said "Yeah, I'll take him for this project." I didn't know for sure how it was going to work out because I didn't know how my project would move his goals forward for sure. But, we did wind up with a negotiation about what he might do for the project that seemed to be something that would be helpful to him.

The two complete mentoring pairs involve the condition of perceived importance from both protégé and mentors. They also demonstrate that the conditions of cooperation towards a shared goal and equal-status within the contact are present in their relationships.

While Eddie and Laura did not tailor the research tasks to better align with their protégés' specific career goals, they were able to identify how the work the protégés were doing connected with their future aspirations. The research that Eddie and his protégé (Jamal) collaborated on resulted in the two presenting at an international conference, which is a fantastic opportunity for any undergraduate. Because these mentors believed mentoring is important and were focused on their protégés' specific academic goals, these relationships were better able to develop a strong interpersonal bond as these mentors made an effort to learn about the protégés' experiences and discuss the significance of the work they were doing. This also contributed to the participants' perceptions that their mentoring relationship was important, particularly for the protégé participants because it was advancing their academic goals. The protégé, Alex, echoes this when he described how his mentor, Steve, took the extra steps to ensure the work 
Alex was doing paralleled with his career aspirations. Alex recognized that Steve used his personal connections to secure "real lab experience" for Alex. This meant a lot to Alex and contributed to why he felt his mentoring experience was meaningful and important.

We realized early on that what I wanted was bench work - like working in a real lab doing real experiments. The problem there is that Steve is handling the policy side of things, so had I done the project he wanted me to do initially, I would have been researching policy and educating legislature about implementing that policy in different institutions and things like that. I'm interested in that, but he empathized with my need to get lab experience. He got in touch with some of his colleagues and the hospital and got me into a lab there where I started doing hands on bench work. It turned into employment, I still work there. And, there's no way I would have ever been able to do that without him. The system is very much a buddy-buddy or a boy's club sort of thing. And, you don't just get that stuff unless you know somebody who knows somebody. I was happy to take advantage of it cause it's turned out really well.

The condition of equal-status was a difficult area to understand in this context of interracial mentoring relationships between faculty members and first-generation undergraduate students in higher education. There is a clear hierarchy between professors and their students, but within these relationships equal-status emerged in relation to how the mentors and protégés worked together, the amount of input each member had on the research tasks, and whose goals were being served. Thus, the most successful mentoring experiences included mentors that engaged in collaborative research with their protégé. This included asking the protégé what parts of the research they most wanted to work onrather than just assigning the most mundane research tasks. When the protégés felt like their input mattered they were more engaged with the work and were able to step into their mentors' role as a lead researcher. Ryan, a black male protégé, exemplified this when he discussed his accomplishments during his mentoring experience. 
She helped me as far as, "Okay, how do you want to present this?" and stuff like that. But as far as the words, they were all my words with little edits here and there that we worked on together. So, pretty much it was all my work, that's what made it more fulfilling because I even googled it and like my name just popped up with Ryan Miller and all their names. And, I'm like "Wow, it's on the internet."

Paul, also a black male protégé, illustrates that working together as a team made the experience more enjoyable.

Like there were things she didn't know and there were things I didn't know. But, then what I enjoyed the most were the moments we sit down and kind of discuss something and kind of say, "Oh, this is not right" or "This is right" or sometimes we would make fun of the data... some countries will actually fudge the data. So, those were the moments I really enjoyed. It was like team work...it wasn't all her ideas and it wasn't all my ideas; so in other words, we were kind of doing team work.

Two other protégés, Maria and Alex, also echoed this same sentiment of feeling like their input mattered in the research process. However, Candice did not mention this process of mutual exchangein her interview, but because her mentor did not participate in the research it cannot be determined with certainty that this process did not occur. From the group of mentors that indicated a successful mentoring relationship, Karen, Julie, Tom, and Eddie all described their mentorship as involving a process of mutual exchange. Laura was the only mentor from this group that did not mention times of mutual exchange in her mentoring relationship.

The presence of mutual exchange fostered a feeling a friendship for three of the protégés, Maria, Candice, and Alex. Both Maria and Candice explicitly reported feeling like their mentoring relationship developed into a friendship, which led to feeling more comfortable with their mentors. Because she now considers her mentor, Julie, a friend, 
Maria explained: "That to me is real...if it was just me and her as me the intern and her as a mentor it would have been kind of hollow." Alex went a bit further and explained how developing a real connection helped him feel more equal with his mentor Steve.

I think after we first met - so it's been over a year now - and we joke with each other and speak really casually, the emails aren't as formal. So, I don't think I feel insignificant or anything like that. We're on even ground I think.

All of the respondents in this successful group highlight how important it is for each member of the mentoring pair to cooperate with one another and find ways to align each others' goals so that each individual is helping the other work towards these goals. This process also contributed to each member's perception that their relationship was important and enhanced their mutual liking for one another. It is likely that in the absence of this process of cooperating towards shared goals, the protégés might not perceive the relationship as important for their own goals. At the same time, this process also fostered a sense of a mutual exchange between the two and increased a sense of equality between the mentors and protégés.

To summarize, the two complete pairs in the successful group are categorized as having the conditions of perceived importance, cooperation towards a shared goal, and equal-status (or a process of mutual exchange and reciprocity), frequent contact, and mutual liking. From the supplemental data group Eddie (mentor) is labeled as expressing the conditions of perceived importance, cooperation towards a shared goal, mutual exchange (equal-status), mutual liking; however Eddie is not considered to have frequent contact present in his mentoring relationship. Paul is found to have the conditions, frequent contact, equal-status, cooperation, mutual liking, and perceived importance 
present in his mentorship. Alex indicates that his mentoring relationship included the conditions of perceived importance, mutual liking, cooperation, and equal-status; however, Alex did have the condition of frequent contact in his relationship. Candice is considered to have the conditions of perceived importance, cooperation, and mutual liking; however, it could not be determined if the conditions of frequent contact or equalstatus where part of her mentoring relationship. Finally, Laura (mentor) described her mentoring relationship as involving the conditions of frequent contact, cooperation, perceived importance, and mutual liking.

\section{The Racial Component}

The interviews for both mentors and protégés asked a few questions that explicitly addressed the racial component of the mentoring relationship. For example, the participants were asked towards the end of the interview, "Did New Horizons do anything that helped you build a relationship with a student/faculty member who is from a different racial or cultural background?" Additionally, the mentors and protégés where asked, "Imagine if your mentor/protégé had not been white/from a minority background, do you think that the experience would have been different at all?" These questions were asked in order to better understand if the participants had considered the racial dynamic present in their mentoring relationship. Not surprisingly, this area of questioning elicited the biggest difference in responses between mentors and protégés. First, I will discuss the mentors' responses and comments about the racial and ethnic aspect of their mentoring relationship. Following this, I will consider the protégés' assessments on the topic. 
The mentors from the successful group provided more in-depth commentary on the interracial feature of their mentorships than the mentors in the less successful groups. Despite this, there were still two mentors in this group (Julie and Laura) that did not consider racial or ethnic differences as being a factor in their mentoring relationships, or relationships with students in general. Their responses to these questions conveyed that they were uncomfortable engaging in a conversation about racial and ethnic differences. However, it could be that both Julie and Laura really do not believe race or ethnicity affects their interactions in a professional setting. In fact, Laura avoided the question about how the experience might be different if her protégé were white all together. When asked specifically about her experience mentoring a student of color, Julie commented, "I didn't think about it.” As a follow up question she was asked, "Throughout the mentorship, did you ever consider the barriers that racial and ethnic minority students face?" Julie replied:

I don't know that I gave it any special consideration than I would for anybody else just on a personal basis. I mean we certainly were aware, but as part of the New Horizons program and we attended all the sessions and...but, I don't think I've ever really thought about it. I didn't think about her differently than anybody else at work.

After a long pause Julie said, "Maybe I should have. It's a good question, I mean." What is interesting about this is that Julie was the only mentor who after reporting that she never considered race or ethnicity did not attempt to explain the justification for this. Instead, Julie took some time to think about it and was able to acknowledge that race and ethnicity might be an important factor to consider when developing an interracial relationship. While I did not consider this to be an indication that Julie was aware of 
implicit racial attitudes or that this demonstrated race awareness, it does suggest that Julie is open to further examining this topic. Both Julie and Laura were categorized as not exhibiting race awareness and not recognizing implicit racial attitudes. On the other hand, mentors, Karen, Tom, and Eddie did demonstrate race awareness and acknowledged that racial stereotypes continue to plague interracial interactions and relationships. Karen and Eddie conveyed the highest levels of race awareness out of all the mentors that participated in this study. These mentors exhibited their race awareness prior to the questions explicitly about race being asked. For example, Karen and Eddie both stressed the importance of providing these types of mentoring opportunities for students from underrepresented groups and creating a more inclusive and welcoming environment for first-generation students. Tom mentioned early on in the interview that it was important for him to consider the status differences between him and Maria, particularly in the beginning of the relationship, in order to make Maria feel more comfortable. Tom explained:

I'm quite a bit older than her, so there is a difference there, and also white male, and so kind of on all levels there's a lot of differences in terms of social status, you know, there are a lot of variables. And, I knew I'd have to, I guess, to make it work, try to, you know, for me to be comfortable with her, to kind of put her at ease and just have some regular contact with her...I just had to kind of keep in mind my age, my degree, my kind of status, that I was male and white, you know all those kinds of things just to try and make it comfortable for her.

Thus, not only does Tom indicate a degree of race awareness, he also implies that group saliency was present for him during their contact with one another.During one of Karen's discussions about developing intercultural bonds she expresses that a number of the conditions from Table 1, particularly those anchored in the contemporary racism 
literature, were present during her contact with Ryan. Karen described how the mentoring program provided a more diverse environment.

Being in a room where people are from a variety of backgrounds makes it easier;rather than, if Ryan were the only AfricanAmerican in that room.It puts a strain on a relationship of somebody withmy background. We had age difference, as well as gender difference, age difference, you know just you name it- we had those differences, but the room was pretty diverse, and I think that makes it easier to make those connections because hey, everybody's different from everybody else in the room. And, I've actually had the experience of being in adifficult situation myself. I was a woman in a...what was essentially an all male undergraduate institution that only had female graduate students...And I've been on the other side of that- where you know, you're the woman walking into this room. You must be the psychologist. Well, yes I am. But you know, you walk into a room- it's all men and you, and immediately they say 'you're the psychologist' because after all you wouldn't be the physicist or the mathematician.So yeah,so I've been on the other side of that. So it'sbetter to be in an environment that is more diverse (to say the least). In terms of doing anything explicit or giving us directions about it, no. That didn't happen. But, the person that is the leader of the program - Alberto - is a wonderful person to create afeeling of warmth and welcoming. Andthat helps as wellto have the leadership be somebody who comes from a diverse cultural background.

In this single vignette, Karen exhibits race awareness, the presence of group saliency, an awareness of implicit racial attitudes (although, not necessarily her own), and uses her experience as a member of a nonracial out-group (women in higher education) to empathize with how it feels to be the Other. This was not the first time in the interview that Karen made the connection between her own experiences as a nonracial out-group member; she also reported that she and Ryan shared similar class backgrounds, as both grew up in a working class family and were the first in their family to pursue higher education. In fact, Karen's father was in the same profession that Ryan was transitioning 
out of. She saw this as a salient common thread between the two, as she mentioned that a number of times throughout the interview.

Additionally, Karen, Tom, and Eddie were the only mentors from the entire sample of mentors that acknowledged how their mentoring relationship might differ if it were a same-race match. Every other mentor either avoided the question or claimed nothing would have been different, at least not from their perspective (one mentor did say it might have been more comfortable for her protégé if he had a same-race match, but nothing would change for her). Both Tom and Karen recognized that if their protégé had been white like them they might not have "tried as hard". For Tom, "trying" was in the context of making an effort to be "sensitive interpersonally" to Maria; put another way, with a white protégé Tom might be more task focused and not concerned with if they felt comfortable or welcome. Similarly, Karen described what she thought would be different if she were a same-race mentor.

In some ways I think I would have learned less. And, especially in a city like this, people have more entrée - they feel more comfortable I think in an environment like the university for example. And...so it...there would have been less of that...maybe I would have done less in terms of trying to connect him up with other people, although I hope not. I hope I would have done as much. But, sometimes it's hard to open those doors and I'm afraid the doors are more open for white people in this city, and maybe even in this college, you know, things do need to change.

Tom and Karen both acknowledge that they perceive an interracial mentoring relationship as requiring more effort on their part and that there are dynamics that must be considered when developing a relationship that bridges racial and ethnic differences. However, Karen mentioned that she too gained from being a part of an interracial 
mentorship and that the benefits to these types of relationships are mutual, a sentiment that Eddie expressed as well. Tom's response was coded as demonstrating an awareness of implicit racial attitudes and group saliency, while Karen and Eddie were coded as exhibiting race awareness, perceived importance, and group saliency.

I turn now towards the protégés explanations to the questions about racial and ethnic differences and how they might affect a mentoring relationship. Four of the five protégés (Maria, Ryan, Candice, and Alex) suggested that having a same-race mentor would have the biggest impact on how they felt in the beginning of the relationship. Other than providing a greater level of comfort upon meeting their mentor, Ryan, Candice, Maria, and Alex did not think having a same-race mentor would change a lot about their mentoring relationship. While Maria and Candice did not further elaborate on this question, Ryan and Alex did. For example, both Ryan and Alex acknowledged that a same-race mentor would likely provide some common ground between the two at the beginning, but they also explained that having a cross-race mentor presented unique benefits as well. Ryan described how this experience contributed to his personal growth.

I mean I think this experience has made me grown so much...just putting me in unfamiliar, I wouldn't say awkward, just unfamiliar positions. Where I'm at, I'm talking to doctors and stuff. I'm just like...I'm doing one at NCC right now, you know. I mean that's how it was, but it made me grow. It's made me grow a lot more in school right now. I mean I was already doing well as far as grades, but this made me like, when I go in front of the class and I speak in front of people, I don't feel that nervousness like I used to feel. So, I feel like it just gave me the confidence to, you know, keep it up and keep going for it. I mean I can say initially maybe my first impression - I would probably have been a little more comfortable, as far as that. But, as far as what I've learned and as far as what I took from being in the positions, I don't think none of that would have changed. I think maybe this position, putting 
me with another person, it challenged me to grow and become more comfortable with other people.

Similarly, Alex points out what he gained from his experience in an interracial mentoring relationship with his mentor, Steve.

I bet I wouldn't have gained as much. I think that meeting someone like Steve brought it to another level. I guess it made me work harder maybe, instead of being comfortable with the same ethnicity;maybe just the potential for advancement. Like just to know that I'm worth more than I thought I was, because maybe someone from the same ethnicity would have just confirmed what I was thinking - that we can only do this much.

All five of the protégés (Ryan, Maria, Paul, Alex, and Candice) indicated that this experience made them feel more positive about connecting with people from different racial or ethnic backgrounds. In other words, the five protégés all explained that they now feel more comfortable about developing cross-race relationships, particularly in the context of mentoring. Candice remarked, "I don't have any uncomfortable feelings or intimidation now at all," about working with a different race mentor. Ryan echoed this when he explained:

I just know that like just because they are not your same race or your ethnic background - they don't match it - that they still can want your best interests and I feel like that's all people really want. They want to be able to trust people and know that these people still want you to really succeed, and I feel like I had that told to me.

Thus, every protégé in the successful outcome group believed this experience was beneficial and contributed to them feeling more at ease in future cross-race mentoring situations. 
To summarize the findings relating to the racial component of these mentoring relationships, three of the five mentors (Karen, Tom, and Eddie) who were categorized as having a successful mentoring experience demonstrated some race awareness. However, Karen and Eddie both conveyed higher levels of race awareness than Tom. These three mentors also shared the conditions of group saliency and awareness of implicit racial attitudes. Karen and Eddie also expressed their perceived importance in regards to their mentoring relationship when discussing the racial and ethnic dynamics of the mentorship. Karen was the only mentor from the entire sample that expressed the condition of experiencing out-group biases because of belonging to a nonracial out-group; in fact, Karen mentioned three different nonracial out-groups she identified with: working class background, first-generation student, and gender. On the other hand, Julie and Laura did not exhibit any of the conditions related to contemporary racism ${ }^{8}$. While Julie did not deny the significance of race during interracial contact, she did acknowledge that she did not consider it. Laura did indeed deny that race or ethnicity impacted her mentoring relationship, as well as with students in general. Furthermore, Laura often changed the direction of the discussion when questions about race and ethnicity arose, indicating that she is uncomfortable engaging in discussions about race (Trepagnier 2010). Thus, I determined that Laura has a low level of race awareness (in terms of how it impacts her interpersonal relationships). For the protégés, four (Ryan, Maria, Alex, and Candice) indicated they would feel more comfortable with a same-race mentor, particularly when

\footnotetext{
${ }^{8}$ These conditions are: race awareness; aware of implicit racial attitudes; mentor has prior experience building meaningful relationships that are interracial or intercultural; and mentor has experience as a member of a nonracial out-group. The condition of group saliency is also included in this group of conditions, although it was not taken from the literature on contemporary racism, this is further explained in the discussion chapter.
} 
beginning this relationship. However, beyond the initial meeting these protégés suggested race did not influence their mentoring relationship. Ryan and Alex even recognized the gains they received by participating in a cross-race mentoring relationship.

\section{Medium Success with Interracial Mentoring Relationships}

I categorized two complete pairs as having the outcome of medium success; Pair \#2 consists of a white female mentor (Jill) and a Latina female protégé (Andrea), and Pair \#3 includes a white male mentor (Patrick) and a Latina female protégé (Renee). In addition to the two pairs, there are two protégés also categorized with the outcome of medium success who make up the supplemental data group for medium success. The two protégés are one black male (Nathan) and one Latino male (Hector).

This section highlights Hypothesis \#2: mentors and protégés who describe a medium successful interracial mentoring experience will be those who expierenced some, but not most, of the conditions from Table 1 . This hypothesis is supported by my data. However, these findings also indicate that the outcomes of these cross-race mentoring relationships are not merely explained by the presence of these conditions, but that both mentoring partners must convey the presence of the conditions, and the conditions are more effective at facilitating a successful outcome when they arise in multiple areas of the mentoring relationship.

\section{Expectations and Perceptions}

This section begins with a discussion about the mentors' motivations and expectations for deciding to become a mentor and what that suggests about the optimal conditions for intergroup contact. Following that, I examine how the mentors and 
protégés describe one another and their interracial mentoring experience and identify what this reveals about the conditions in their relationship. While most of this discussion will center on the two complete pairs, the two protégés from the supplemental data group will be referenced occasionally to further support the findings or highlight a nuance not discovered from the two complete pairs.

The two mentors, Jill and Patrick, both provided a number of different reasons for deciding to be a mentor. For example, Jill explained all the different research projects she had going on and how they "have generated stacks of articles in my lab, which Rick [a minority staff member who Jill worked closely with] was not able to coral and get under control, so when the programs' solicitation came out," she thought Rick could use the help. Later on in the interview she again mentioned Rick, but in a different context, as being her motivation for being a mentor.

Well [Rick]...He is a scholar of color. And he is lonely as heck, and I don't like him having to be lonely as heck; so, when this came up I thought, oooh, let's see whether somebody who would not be a peer, but still would be somebody else to... and see how he feels about the possibility. He was interested, and he thanked me for it, which I thought was a little unsettling... Okay, so my dad taught at a HBCU (historically black college or university) and so I really noticed...I mean that's not the only thing, but you know...the only reason. But I really noticed how incredibly white this university is, at the faculty and administrative levels. And we're missing a lot of perspectives that we need, so I feel like I would like to be part of a larger project to bring more people of color on board.

While Patrick's motivations did not include contributing to a more diverse institution, he too mentioned reasons other than his research agenda, although his researchwasalso a factor in his decision. He explained, "I mean I think in my mind I was half thinking that 
I'm doing this program half because I think I might be able to get some research assistance out of it, and half because I thought it was a good program and I would give it a go." Further on in the interview, he was asked to elaborate on why he thought the program was good. Patrick answered

Right. There are probably three streams. One is I'm currently funded by [the same institution as New Horizons is] so I feel beholden to those programs. So there's that. And then obviously you're always looking for someone to help out in the research and get work done. But like I said, I have to say going into the program I knewin my heart of hearts that it was probably going to end up more of a net loss of time than it was a net gain of productivity. It didn't turn out to be that way. I was pleasantly surprised. But you know I didn't know too much about the New Horizons program beforehand; just kind of from what the PR was out there- that they were bringing something like lower SES or disadvantaged students, trying to bring them into the university and research domain. And I thought that was a good project.I didn't do it for the money, cause I didn't even end up claiming the money at the end. I just think it's important to get younger people in the college scheme of things more involved in research.

Both Jill and Patrick highlight their own research agenda as important reasons why they chose to be a mentor. At the same time, they both explain their choice as being motivated by something bigger. For Jill, she mentioned contributing to a more diverse institution, which does demonstrate some race awareness. However, the focus is placed on Rick and not her protégé Andrea. Similarly, Patrick does not really mention any goals for his protégé Renee. Interestingly, when both mentors were asked if their mentoring experience met their expectations and goals their answers only related to the mentors' research agendas and how Andrea and Renee contributed to it. Patrick reported that the experience actually "exceeded" his expectations. He went on to say: 
When we did it next time I might even try to see if I can have two students or three students. I mean I know there needs to be more going, to go around; but because you're going to spend all the time and effort to train, and advise, and mentor one, you might as well train, mentor, advise three. And then you actually do make more of a...you sort of recoup more. Although, it's still true that those other two may be complete duds.

Along those same lines, Jill answers the question about her experience meeting her goals and expectations with:

Yeah. I would say about $95 \%$ in the sense that we got rid of the stack of un-entered bibliography items, which is huge for me. The 5\% that's missing is that it turns out that when the database spits out citations, they are not all in the same format now. I don't understand how that happens. So, I'm not quite sure... so now my grad student spits them out of the database and then spends all kinds of time in Mircrosoft Word fixing them. And that's not what it was supposed to do. But, I feel like, "Well, you know, this is the one thing in my lab that I don't want to have to micromanage. And, this is the price I pay!" I have a tendency to micromanage too much, and this is why. But yeah, in general, I think it went really well.

The two follow-up questions asked Jill about her specific goals for Andrea and if Andrea was transitioning into the university. To the former she replied "No" and the latter, "Oh, I think so. Yeah, I don't know too much about that." These mentors indicate that learning about their protégés' academic and career aspirations was not a priority when choosing to be a mentor, or when evaluating their mentoring experience. Therefore, both Jill and Patrick do not fully confirm the conditions of perceived importance or cooperating towards a shared goal when discussing their motivations for mentoring.

All of the respondents were asked to describe their mentoring partner, and later were asked if their impression of their partner changed throughout the relationship. These questions helped to determine if the pairs shared a mutual liking between the two and if a 
personal connection developed throughout the mentorship. Five out of the six participants (Patrick, Renee, Andrea, Hector and Nathan) that are categorized with the outcome of medium success described their mentoring partner in a positive manner. Jill, one of the two mentors in this group, was the only participant that I did not label as describing her partner in a positive way; however, this is not to suggest that Jill only used negative descriptions when discussing Andrea, but that it was a mix of positive and not positive descriptions. Jill described Andrea more in terms of how Andrea could contribute to Jill's research than in respect to Andrea's personality.

I remember that Andrea seemed to take it seriously in a way that impressed me favorably. She understood that it was an interview that it wasn't just dropping in to be friendly...she seemed open to being ready to participate and help out in a range of ways, and that mattered a lot to me because I didn't want to have to worry about whether somebody thought the work was below them.

Jill was then asked to elaborate more on her first impression of Andrea. Jill responded:

Quiet, naïve, curious, and kind of...maybe limited in her education. Well, I already said naïve, but more like, she didn't have a whole lot of...I mean she's a community college student what could she have?

Jill also noted that Andrea was "hard to get to know" and "informal in her dress." From Jill's responses, I did not feel like I could confidently code Jill as having developed a mutual liking or personal connection with Andrea. On the other hand, Andrea's description of Jill was much more positive and centered on Jill's personality. Both Patrick and Renee described one another in positive ways, yet their descriptions suggested that the two had not developed a close interpersonal bond, but that mutual liking was present between the two. 
Similarly, the two protégés, Nathan and Hector, from the supplemental data group both described their mentors positively but also indicated that their relationships were very centered on their work with their mentors and that they did not know much about their mentors beyond their education and research accomplishments. Although, when Nathan described his mentor, Brian, one of the first things he said was, "I don't think he has...I don't think he's a racist at all.” Thus, Nathan's comment suggest that as a protégé of color, it was important to him to determine that his mentor was not racist and that Nathan was aware of the potential of having a mentor that he perceived as racist.

All of the protégé participants were asked how they felt upon meeting their mentor for the first time. For example, did they feel self-conscious during the first meeting? Did they pick up on any similarities or differences right away? Three (Renee, Andrea, and Hector) of the four protégés in this medium success group described feeling nervous or self-conscious when they met their white mentor for the first time. Nathan was the only protégé that made an immediate connection with his mentor right away. Although Andrea did say there was an immediate connection, her explanation of the connection does not indicate that there really was a connection right away. Andrea said, “I guess so. I didn't feel like, 'Oh God! I'm going to be working with this woman!” Additionally, she mentioned that if Rick, the minority staff member, had not been at the first meeting it would not have been as comfortable for her. None of the four protégés described picking up on any similarities or commonalties between themselves and their mentor right away. However, three of the protégés (Andrea, Hector, and Nathan) mentioned their racial, cultural, and educational differences as standing out to them at 
their first meeting. Hector explained that he felt like his mentor, Lisa, had different lived experiences than himself.

Given my, you know, the many identities I have... in terms of like being a communication major... but, yes. I'd say not too different, but somewhat different because I'm Latino and she is...she was...she is white. You know, the education difference as well...like, you know, being Mexican. I identify myself as Mexican-American. I have...it's like, you know, that's all a lot of different things and traditions and things and are completely different in that aspect. There's some differences in terms of the people I socialize with normally outside...like professional settings or things like that. Maybe different lived experiences as well.

Renee was the only protégé that said she did not feel like there were a lot of difference between herself and her mentor, Patrick. On the other hand, the mentors, Jill and Patrick, did not feel like their cultural or racial differences stood out when they met their protégés. For example, Patrick said, "For me, sort of the notion of cultural/national differences was very, very, very low on my radar screen. I mean I was really looking for someone, you know, can you do the work? Can you do it well?" Both Jill and Patrick said there was not an immediate connection on a personal level, because that's not what they were looking for.

To recap, both mentors, Jill and Patrick, indicated that they lacked the condition of perceived importance in regards to how the mentoring relationship would benefit the protégés' academic and career goals, and instead were more focused on their research needs. While Jill did indicate some race awareness it is unclear how she saw this experience benefiting her protégé Andrea, and directed her race awareness at contributing to a more inclusive environment for her colleauge, Rick. Patrick's comments suggest a 
low level of race awareness. However, three of the four protégés (Andrea, Hector, and Nathan) in this medium success group implied that the racial and cultural differences between themselves and their mentors impacted how they felt, particularly in the beginning of the mentorship. While Patrick and Renee indicated mutual liking for one another, they also both claimed that they did not develop a real connection. Because Jill explained that there was not a real personal connection between her and Andrea, I labeled this pair also as not having a personal connection. Thus both mentoring pairs were categorized with a low level of mutual liking that is not accompanied with a strong personal connection. Nathan is the only respondent in this group that I determined made a real connection with their mentoring partner; however, because he is from the supplemental data group his mentor, Brian, did not participate in this research and this condition cannot be confirmed among both members of the mentoring pair.

\section{The Mentoring Relationship}

This section is organized according to how much time the protégés and mentors spent with one another, the activities and work the pair engaged in, and if and how the mentoring experience contributed to the protégés' academic aspirations. The respondents' descriptions concerning these aspects of their mentoring relationship help to determine if a number of the optimal conditions for intergroup contact are present or absent. For example, the conditions of frequent contact, perceived importance, cooperation, and equal-status within the relationship are some of the conditions most emphasized in this section. I will also highlight specific conditions that only emerge in a small number of the participants' responses. 
All of the participants were asked about their typical routine throughout the mentorship and how much of their time was spent interacting with their mentoring partner. Patrick and Renee were the only mentoring pair that indicated they had frequent contact with one another. They met at least once a week during their summer mentorship and have since continued working together for almost a year, still continuing to meet either once a week or bi-weekly. Therefore, Patrick and Renee are labeled as having frequent contact. Conversely, Jill and Andrea did not meet the criteria for frequent contact. While Andrea did report that she had frequent contact with her mentor it was unclear if she was referring to frequent meetings with Jill or with Rick, the staff member. Ultimately, I categorized this pair as not involving frequent contact, because Jill was explicit that she did not spend a lot of time with Andrea and that most of Andrea's time was spent with Rick. Jill explained:

Andrea met with my staff assistant every day, every day that she came in. She didn't have a key to the lab herself, so my grad student had to be there. But, if my grad student hadn't been there I would have met with her more. So, it's not like...I don't want to give the impression that I abandoned her.

Similarly, Hector reported that he spent most of his time meeting with his mentor's research partner, not his mentor Lisa. He also mentioned that he did not have as much direct interaction with Lisa as he had originally expected, that she was extremely busy, and that he wished he could have had more support during the poster presentation ${ }^{9}$. Nathan also indicated that he only had face-to-face contact with Brian "once or twice a month, but not much more than that." Most of their contact was through email or phone

\footnotetext{
${ }^{9}$ For a description about the poster presentation please see the second paragraph of this chapter, as it is discussed in detail there.
} 
conversations. Therefore, Pair \#2 (Jill and Andrea), Hector, and Nathan were all labeled as not having frequent contact. Additionally, Hector's comment about not receiving as much support as he wanted suggests that the condition of cooperating towards a shared goal was not present.

I now focus on the research tasks the protégés did and if they perceived it as important and contributing to their professional development. Additionally, I identify how much effort the mentors gave in respect to their protégés' personal academic and career goals. In some cases, how the participants discussed this aspect of their mentoring relationship also conveys if there was a sense of equal-status between mentor and protégé, and the level of cooperation that occurred throughout the mentorship. Equalstatus was reflected when both participants described having equal input on deciding tasks and that both members' goals were considered and promoted by the research tasks. Three out of the four of the protégé respondents (Renee, Hector, and Nathan) in the medium success group described their research tasks as contributing to their professional development. Renee and Nathan both explained that their research experience fostered a growing passion for research and both plan to pursue a career in research and have greater confidence in their skills. While Hector did not mention that his career goals include pursing research, he did indicate that he felt he was able to significantly contribute to his mentor's research and this increased his confidence in his academic capabilities. This suggests that Renee, Hector, and Nathan all perceived the work they were engaged in as important to their personal academic goals. The actual research task that Andrea worked on during her mentorship did not appear to be perceived as important to either Jill or Andrea, in the sense of enhancing Andrea's research skills and 
knowledge. The only research task that Jill discussed in terms of Andrea's contributions was organizing a bibliographic database, which she also mentioned would have been Rick's (her colleague) responsibility if she had not been a mentor to Andrea. This indicates that Andrea and Jill did not establish a level of equal-status within their relationship, as Andrea never had the opportunity to step into the role of a lead researcher. Conversely, Patrick did make an effort to create research tasks for Renee that allowed her to experience what being a lead researcher entails; thus, their relationship did involve some level of equal-status in respect to the research tasks. Indeed, Patrick explained that it was important to him to provide research work for Renee that was engaging and fun.

You'd hope that the faculty members would try to not just use the students as a vehicle for enhancing their own program, but would actually take the pedagogical side of it seriously and try to make it fun for these students to get them...cause it...any research project (from my perspective) can be made interesting.But, if you're just giving a bunch of stuff to you know... wrote stuff, hunt down, or whatever - I don't think that serves the project or the program or the student any. And, I heard you know sort of by the grapevine, Renee would tell me things like you know some of the students weren't happy cause all they were doing was just literature reviews. They were just doing literature reviews. And it wasn't...all they were doing was photocopying.

At the same time, Jill and Patrick did not make an effort to learn more about their protégés' (Andrea and Renee) academic and career aspirations or go out of their way to ensure that they helped with the transition from community college to the university. Similarly, neither Hector nor Nathan mentioned anything their mentors did for them outside of giving them research work. Therefore, the two complete mentoring pairs and 
the protégés from the supplemental data group are not categorized as establishing the optimal condition of cooperation towards a shared goal.

Another area where some mentoring pairs were able to establish cooperation, perceived importance, and/or equal-status is the poster presentation the protégés created and conducted at the end of their mentorship. Equal-status emerged in this area in the ways the protégés described the amount of attention and emphasis the mentor placed on helping the protégés meet this goal of designing a professional poster presentation. Additionally, some protégés also indicated that working on the poster presentation with their mentor was a collaborative process (equal-status present) while others explained their mentor took over when helping the protégé with this task (equal-status not present). However, in this group of participants (medium success) the only mentoring relationship that suggested optimal conditions were present was Pair \#3 (Patrick and Renee); but, because Patrick made only one reference to Renee's poster presentation I cannot confirm if perceived importance or equal-status was present in this portion of their relationship. Renee did indicate that Patrick did work with her to create her poster.

He's really supportive. He really helped me especially to, like, put my thoughts together when we had to do the poster presentation because I was like, "Okay, I have all this information or this data. Where should I focus on?" And he really helped me to like... what to focus and how to do the poster and [had me present for him] again and again. So, he was really helpful.

While Andrea did not discuss if and how much Jill helped with the presentation, Jill did explain that she only "saw a few graphs and then was gone" and that it was Rick who helped guide Andrea through that process. Hector felt like he did not have enough 
support from his mentor when he was creating the poster presentation, because she was so busy. Nathan's experience was quite the opposite from what Hector described. Nathan explained:

I did that independently. And there was a problem. This is when I began to realize that this was going to be an issue, us working together, because my mentor was so hands on that I felt like that the poster wasn't going to be mine, it was going to be his, so I started to push back. And I ended up doing something at the last minute because I didn't want to be nit-picked,you know. And then I think one of the other issues was they didn't send me...I didn't have a really good guide to start with. I didn't have an example of what they wanted. So I started on something on my own; it wasn't what they wanted, so that was just creating more and moreissues between us because I finally decided it's gonna be his and it's not gonna be mine; so, I'm just gonna like put it on the back burner because I had fall classes and I had a lot things going on with school. Yeah. So I just basically didn't want to do a poster that wasn't going to be my own. But I ended up getting one that was my own. And it was kind of tough to try to keep my own voice for it.

Nathan's experience during the poster presentation part of the mentoring relationship demonstrates a lack of cooperation and equal-status, while Andrea and Hectors experiences with designing their posters suggest a lack of perceived importance, cooperation, and equal-status between mentor and protégé. The condition, equal-status, was determined as absent because the mentor did not put equal emphasis or consideration on helping the protégé when it was not related to the mentor's research agenda.

To sum up, only one complete mentoring pair (Patrick and Renee) in the medium success group met the condition of frequent contact. Additionally, among the two complete mentoring pairs, only one pair (Patrick and Renee) established equal-status, cooperation, and perceived importance in their mentoring relationship; however, these 
conditions were not present in all areas of their relationship or at all times. Therefore, Patrick and Renee are labeled as involving a mixed bag of the optimal conditions: equalstatus, perceived importance, and cooperation towards a shared goal. Jill and Andrea are categorized as not establishing the conditions of equal-status, cooperation, and perceived importance, although Andrea did at times indicate perceived importance. However, Jill did not, the condition is considered to be absent. Finally the supplemental data group, consisting of two protégés, Hector and Nathan, also described a mixed bag of the optimal condition of perceived importance and did not indicate that the conditions of equal-status or cooperation were present in their mentoring relationships.

\section{The Racial Component}

This segment is arranged according to how the two mentors, Jill and Patrick, described the impact of race on their mentoring relationship, which determined if the optimal conditions of group saliency, race awareness, aware of implicit racial attitudes,

prior interracial relationships, and identification with a nonracial out-group were present, absent, or sometimes present in the mentors' interracial mentoring relationship. Following this, is a discussion about how the protégés explained the impact on race in their cross-race mentorship and how this relates to the same optimal conditions.

When Jill and Patrick were asked how their protégé identifies racially or ethnically, it was clear that neither of the mentoring pairs engaged in conversations about their protégés' racial background and experiences. Jill answered the question by saying, "I assume as Latina. I never asked her." Patrick did not explicitly say that he and Renee never talked about her ethnic background, but when he was asked about her ethnicity he 
could not remember where she was from. Another interesting aspect about how Patrick discussed Renee's ethnic background is that throughout the entire interview he never referred to her Latina background, instead he always referred to her as an international student and he talked about their national differences not ethnic or racial differences. Patrick also indicated that for him, group saliency was not present in their relationship.

I mean there's the issue of can you understand me. But was it very clear from the very first meeting that she could understand and be understood. You know, yes she has an accent and all that stuff. But from my perspective, it was never...I never thought in my mind that I'm doing this for international students. I didn't really even feel...I didn't really know that that was like a MO for New Horizons from the get go. I knew it was like disadvantaged students, but I didn't know that it was necessarily or had to be international students. So for me it was always just like I said it wasn't really a big issue for me.

Patrick did not respond to any of the questions about race and ethnicity in a way that suggested he was aware of their racial or ethnici differences. His statement implied that he perceives a qualitative difference between racial/ethnic minorities and immigrant minorities, an important distinction within the social science literature as well (Portes 1999; Portes and Zhou 1993). For example, racial prejudice is a distinct feature of US society; thus, many immigrants enter the United States without knowledge of, or experiences with this form of discrimination (Portes and Zhou 1993). It could then be the case that Patrick does not understand this relationship as an interracial relationship, but rather an intercultural relationship. Therefore, I can only confidently determine that Patrick did not consider their cultural differences as impacting the relationship and cannot label the condition of race awareness as either present or absent. 
Only once did Patrick indicate that he was aware of the potential forcultural stereotypes to arise; however, in the same comment he also explained that he was unlikely to give into stereotypes.

I mean I think that we're prone to stereotype. So you know it's possible that I could say something...I don't believe this...but, I could actually say something like "well you know, because she's from a different culture, she respected power more. And therefore she was more deferential and professional." But that might just be a load of hogwash. I mean I've worked with people from, you know, other...other international students as graduate students who are massively entitled and pains-in-the-butts. And, so I think, you know, I don't think it really affects my future relationships with them in that sense. Cause at least...with what I was doing there wasn't any sort of tension between, you know...that was caused by the international angle.

When asked how his mentoring experience might have been different if his protégé had been white like himself, he suggested that he might have been able to read her (the imaginary white protégé) a little bit better and created less feelings of “I don’t know her. I don't know what I'm getting into." But, that it would not have "changed anything about the relationship." It appears that Patrick was implying that he would have felt more comfortable, particularly at the beginning of the mentoring relationship, if his protégé had been white. However, his answer again indicates that the biggest differences were their cultural and national differences not racial/ethnic differences. Therefore, as the researcher, I feel that applying his statements to the conditions that I proposed based on contemporary US racism is not a legitimate categorization. I did not code Patrick then for indicating the presence of the condition, aware of implicit racial attitudes. The only condition I can confidently determine from his discussion is that group saliency was not present for Patrick. 
Jill was more forthcoming about discussing race and ethnicity, and demonstrated

some race awareness. However, she avoided the topic of the racial dynamic between

herself and Andrea and never indicated being aware of her own implicit racial attitudes.

In fact, when Jill did express race awareness it was usually in reference to how most

other white people in the university and surrounding area lack any kind of race

awareness.

It really seems to me like there's very little here for students of color. There are student organizations, but like there's no real way that grad students or undergrad students of a particular...particularly minority ethnic background, and let's just even say particularly underrepresented that can collect around common interests. And so these common interests could be about, you know topics or something else that we don't anticipate because we're not them. So I just really think that the faculty at this institution needs to find out that they're missing a lot. And it really...I think the...particularly at the faculty meetings, when we're trying to figure out how to distribute some scarce resource, a faculty member, such as myself for example, can raise the question "oh and can we think about whether we want to use... bringing more underrepresented groups aboard as part of this." And people nod and go "oh yeah that would be good." And then they go on, and they think they're done. Because they just had an emotional experience of how nice that would be and they are done they think. It gets to the point where it's like almost not worth raising it, because all it is is "oh, there they go again. Okay, we're going to smile and nod, smile and nod. And then we'll be done. We just have to endure this statement from this colleague." Things like, "gee it's really too bad there weren't more qualified scholars of color in this applicant pool," or whatever. What's it gonna take for people to figure out they're missing something? So to me, that's where this mentoring program fits in. And by the way, I'm not besmirching my department in particular. I'm just trying to, you know I'm trying to describe what I think is a broader issue. And I don't know what it's gonnatake to address it, but I love it that when I go to a mentoring thing and I look around and I see who the other faculty are, I feel like okay whatever those faculty members own ethnic backgrounds are, I've got something in common with them. You know, and I like that very much. 
In the above statement, Jill does exhibit some race awareness, but at the same time she only discusses how hard this is for her when her white colleagues are not concerned enough with the lack of diversity within the institution. Moreover, when she talks about the contributions the mentoring program is attempting to make by creating a more racially inclusive university, she never mentions the students, only that she feels good being in the same room with other racially aware faculty members. Additionally, Jill was asked if there was anything the mentoring program could or should do to strengthen these interracial mentoring bonds between the faculty mentors and student protégés. She responded with a quick "No," and, instead went into a detailed explanation about what the mentoring program should do is address things like Andrea's poor writing skills. Then she explained that there were no cultural barriers between her and Andrea, which was then followed with another suggestion; that the mentoring program teach the protégés how to appropriately dress when their mentor invites them to meetings with community partners, as this was an issue for her with Andrea. She also explained that Andrea's casual dress was not cultural, "but more a socializing thing." Jill then said, "I don't really see that as cultural for the record. I see that more about maybe a firstgeneration issue." Thus, Jill's level of race awareness is at times difficult to assess. For example Jill acknowledges the lack of diversity within University of the Pacific Northwest, but also demonstrates what Bonilla-Silva (2003) labels as color-blind racism when she points to Andrea not being "socialized" to know how to dress appropriately in a professional environment, but then following that up by implying that that has nothing to do with her Latina background and is instead a result of her first-generation status. Thus, 
the statement becomes confusing as to what Jill means. In the end, I labeled her as sometimes demonstrating race awareness, but not a high level of race awareness.

Out of the four protégés, three (Andrea, Hector, and Nathan) all mentioned their racial and ethnic backgrounds as impacting their mentoring relationship. For example, Andrea explained that she developed a much closer relationship with Rick, the staff member, than with Jill.

I spent a lot more time with him (Rick) and we just had a lot of things in common in terms of social-economic status, dealing with racism, and experiencing racism, and like I was definitely closer with him. I would say probably [because] of the race factor, cause we had a lot of conversations about that. I felt like that really...I don't know. It's always nice to feel the shared...like your experience with another person who has shared... who have experienced other forms, or similar forms.

This was a recurring theme with Andrea, Hector, and Nathan. All of them mentioned that if they had been matched with a same-race mentor they would have felt more comfortable and shared some same lived experiences. At the same time, all three of the protégés explained that their mentors were all culturally competent, but that was most likely not the case with all the other white mentors. Additionally, all three protégés mentioned being aware of how inequitable the world of higher education is and that they need to be comfortable working with white mentors and faculty members, because "that's the way it is." Andrea, Hector, and Nathan all exhibited high levels of race awareness and group saliency was present to them during their mentoring experience. Conversely, Renee did not express race awareness or indicate group saliency in her mentoring experience. 
Similar to her mentor Patrick, she gave the impression that she did not have the experience, or the identity, of a racial minority and does not treat herself as "nonwhite"

In sum, I categorized Patrick and Renee's mentorship as not being applicable for determining the presence or absence of the conditions: race awareness, aware of implicit racial attitudes, and membership in a nonracial out-group. However, I did code Patrick and Renee as lacking the condition of group saliency. The only condition that was present was prior intercultural interactions; however, it was not a strong condition as it was hardly mentioned by the two. Jill and Andrea were labeled as having race awareness, but not a high level; and group saliency as present somewhat. The conditions of aware of implicit racial attitudes and membership in a nonracial out-group were not present in Jill and Andrea's mentorship.

\section{Low-to-No Success in Cross-Race Mentoring Relationships}

This section describes how the participants who were found to have low-to-no success with their interracial mentoring relationships discussed their experiences and perceptions surrounding this relationship. It is organized according to: (1) expectations and perceptions; (2) the mentoring relationship; and (3) the racial component.The two complete interracial mentoring pairs that are considered as not successful mentoring experiences involve Kathy/Jarrod and Melissa/Miranda. The first pair (\#4) consisted of a white female mentor (Kathy) and a black male protégé (Jarrod); the other pair (\#5)

\footnotetext{
${ }^{10}$ For more on the differences between racial/ethnic minorities and immigrant minorities see Portes (1999) who delineates these differences and explains that in the US, racial minorities are involuntary minorities and go through a socialization process, in which they are informally and formally taught how to interpret and cope with racial discrimination. On the other hand, immigrant minorities are voluntary minorities and do not undergo said socialization and have a dual frame of reference with which to interpret their experiences. Also, see Portes and Zhou (1993) for a more in-depth explanation on
} 
included a white female mentor (Melissa) and a black female protégé (Miranda). There were not any participants from the supplemental group that indicated an unsuccessful mentoring experience; however, I cannot say with certainty that their mentoring relationship was not unsuccessful. It could be that if their mentoring partner had participated in the research my analysis of their relationship would be different. For example, with Pair \#4 (Kathy and Jarrod) it was only Kathy, the mentor, who explicitly reported the relationship as unsuccessful; thus, with only Jarrod's participation I would have had a difficult time determining the outcome of his mentoring experience.

The following discussion addresses Hypothesis \#3: mentors and protégés that describe an unsuccessful mentoring experience will not have any, or very few of the conditions from Table 1 present in their relationship. The hypothesis was supported from my data, as the mentoring pairs with a low-to-no success outcome were not able to establish many of the conditions. Additionally, the few conditions that were present were not present in conjunction with other conditions in order to build on each other and strengthen their impacts.

\section{Expectations and Perceptions}

Determining what the mentors' expectations and goals were for becoming a mentor offer insight into how important it was to the mentor to develop a bond with their protégé and if they were concerned with advancing the protégé's academic and career aspirations. The mentors in this group expressed very different motivations for joining this mentoring program. First, Kathy's expectations and motivations were very much 
centered on her research agenda and little importance seemed to be placed on developing an interpersonal bond with Jarrod, her protégé.

I was kind of at the point where I was working on my research and that was really the priority for me. I was thankful Jarrod was able to give me some help, but also I wasn't too concerned about, you know, do we like each other?

Later in the interview when Kathy was asked if her mentoring experience met her goals and expectations, she still did not mention having any specific goals for Jarrod and again illustrated that her priority was her research.

You know, I really thought it would be cool to meet someone who was interested in the project and someone who might want to be more involved in it and really understand more of what was going on. I thought it would be really cool to have someone that would want to come to a conference with me, someone who could really be a contributor. So in that way, no; but, I thought, well it would be nice to get another pair of eyes on the references and get the data entered, that really helped. And, I think he did a good job with that, but that is where it ended I think.

The reasons Kathy gave for deciding to be a mentor, suggested that she did not perceive this interracial contact as important. What was most important to her was having an "extra pair of eyes" to assist with her research, regardless of whose eyes they were. Her response also indicated that it was unlikely that both her goals and Jarrod's academic goals would receive equal consideration within their mentoring relationship; thus, foreshadowing that the condition of equal-status will not be established. In fact, when Kathy was asked if she thought she was able to help Jarrod with a smoother transition to the university the only thing she mentioned was that she checked out a book for him that was related to his major. 
On the other hand, Melissa emphasized this relationship as an opportunity to create connections and positively impact a student's life. She also highlighted the goals of the mentoring program as a motivator for her to become a mentor.

It just seems like this beautiful ideal at the moment that you've been waiting for that frankly, in this institution, we don't get a lot of opportunities to have. It gives you the idea that what we're doing isn't just coming to work every day and regurgitating something to people, but you can have connections; you have the potential to make impacts on people's lives...More importantly, I think the biggest asset to the program is the transition from community college to regular university life. I believe it's my responsibility as a member of this community to be an active member and that was a way that I saw to do that.

Melissa expressed that this relationship was important to her and she hoped to contribute to the academic goals of her protégé, Miranda. Although this relationship did not turn out to be a success, Melissa does demonstrate that, at least in the beginning of the mentorship, she did perceive the contact as important and expected to develop a close interpersonal bond with Miranda.

All of the participants were asked about their first impressions of their mentoring partner and later asked if their impressions changed over the course of their mentorship. These questions provided information about whether there was mutual liking between mentoring pairs for the four respondents in this section. Three (Kathy, Melissa, and Miranda) out of the four participants in this group indicated that there was difficulty developing a personal bond between mentor and protégé among these pairs. While Jarrod did say that he felt he connected with Kathy, his explanation seems to suggest that a strong connection between the two did not actually develop, which Kathy confirms in her interview. Jarrod reported that they connected from the beginning and said "I guess I 
consider her a friend now." He was then asked to talk about what made the connection

real to him.

I think it was when...because I'm really shy and have trouble talking to people sometimes. And, in the beginning Kathy would always like ask me questions and stuff; but, as the summer progressed she started to like give me more space and would just...like she wouldn't ask too many questions. Like she would ask something and then if I said "yes" or "no" that would be good enough for her.

Kathy also described Jarrod as shy and timid, and for Kathy this was a major reason why the two did not connect. In fact, Kathy indicated that at times their personality differences created an uncomfortable dynamic between the two.

I remember when I took him on interviews one day and I thought oh this is a chance to...I'm going to be able to, you know, talk to him a little bit more about outside things. So I said, "So what do you like to do in your spare time?" And he just sat there and said [in a lower voice to mimic a male voice] "I like to play video games." And that was kind of all. And I remember feeling really strange. I look back now and I guess it was funny, but I just thought okay this is strange.

Both Jarrod and Kathy reported that the two did not share any common interests and that their starkest differences were their personalities, with Jarrod being extremely shy and Kathy being an out-going extrovert. Although the two both described the other as nice and smart, it was apparent that there was not a strong connection and they knew little about the other person.

Turning towards Pair \#5, Melissa and Miranda, it was clear that they too were lacking the condition of mutual liking, particularly towards the end of the mentorship. Melissa explained that upon first meeting her protégé, Miranda, she had a positive impression of her and had high expectations that their mentoring relationship would 
continue after the program ended. At the beginning of their relationship Melissa

described Miranda as "positive...very kind, and honest." Within the first few weeks of their mentorship Miranda shared with Melissa some of the life struggles she was going through at the time, and Melissa felt like this personal sharing brought the two close together. However, this quickly changed according to Melissa.

I feel like that if anything...I felt that we were more connected because she shared that information with me and I worked hard to facilitate flexibility with what might be going on her life, and an opportunity for her to share this open communication about the struggles. But, she, in my opinion, started to take advantage of that and actually then our...I felt like our relationship deteriorated because of that.

Miranda did not mention this moment of sharing between the two, but did mention the life circumstances that she was going through during her interview. In fact, Miranda could not recall Melissa's name when asked at the beginning of the interview. Miranda also commented that, "going in, I did feel a little intimidated only because she was so academic heavy, like I did feel judged to a degree." This indicated there was not equalstatus present in their relationship, as Miranda felt she was looked down upon. In addition to this, Miranda described the mentorship as a job, and never thought of Melissa as her mentor and did not feel like any mentoring occurred. Melissa also commented that her impression of Miranda's motivations for joining the mentoring program had less to do with developing a mentoring relationship and more to do with enhancing her resume.

It felt often times like she was faking the process; she did a lot of talking and not a lot of showing. I almost felt like she engaged in the program because she thought it would look good on her resume as opposed to that she actually wanted to do it, which I think you get different results from that.

Melissa also added 
I just felt like I invested a lot in this and I had a lot of expectations, high expectations, for the way it would turn out. So, I felt really frustrated that it didn't go that way, but I felt really let down as well that this person obviously was not who I thought they were and that they didn't care, they didn't have respect for me and what I do because they left me high and dry. I just felt...frustrated, let down, maybe even hurt that I chose to this and I chose to invest my time in this and this person obviously didn't have the same expectations.

Between the mentor, Melissa, explaining that she felt taken advantage of by Miranda, and the protégé, Miranda, reporting that she never thought of Melissa as her mentor it is clear that this pair did not involve the conditions of mutual liking, clearly defined roles, or cooperation towards a shared goal. Both of the participants indicate the lack of these conditions throughout their interviews. Additionally, Kathy and Jarrod are not coded with the condition of mutual liking as present or absent at this time (this condition is further discussed below). This pair was also categorized as lacking perceived importance and cooperation.

\section{The Mentoring Relationship}

When these participants answered questions about the work the pairs did and how mentor and protégé interacted with one another it became clear that there was little cooperation or mutual understanding between Pair \#5 (Melissa and Miranda). Moreover, Miranda's comments about why the two did not develop a real connection underscore the significance of perceiving the contact as important. Miranda noted that

I could have developed a stronger connection in the sense of looking at her (the mentor) as a future colleague or someone that I might utilize to write me a letter of recommendation; that sort of connection. But as far as connecting on a personal level, maybe I just didn't desire that. I didn't find the need I guess. 
Although Melissa does suggest that the two had a meeting in the beginning of their

relationship to outline one another's expectations and Miranda's tasks for the summer, Miranda reported that she was unclear about what was expected of her. Below, Melissa describes their first meeting together.

Our first meeting in person was at my office. We kind of just talked about what the goals were for the experience and what kind of project that she would be working on. Then we set up a plan that would basically start once the contract started. The way I approach those kinds of sessions are always like, "Here's what I need. What do you think you need and want?" And, I don't remember the details of the conversation, but I know that she had already expressed to me the interest, even after the data entry, which I consider extremely boring, wanting to be on the project and saying, "Well when I become a [part of this] major maybe I can still work on these projects with you."

In contrast to Melissa's comments above, Miranda explained that

After the data entry I really wasn't aware what...of what was expected of me, other than researching a ton of journal articles that were related to environmental justice, but that was all mundane and just...I don't know if I did it right or not, lack of feedback.

Miranda seemed to contradict herself later in the interview when she explained that her and Melissa did talk about what Miranda's tasks and responsibilities where going to consist of. At the same time, Miranda again indicates that she did not perceive Melissa as her mentor, but rather saw this experience as an opportunity to gain job experience.

We did go to lunch, and that was, I believe, her attempt to connect. But, it was very driven to her research. It was...to me it felt like a job. To me it was...I didn't see her as the mentor. It was, "Okay, these are the tasks at hand. This is what you need to do. I guess I didn't look at her as...she wasn't my academic mentor; she was more like, okay let's get experience in a job setting. 
Upon both Melissa and Miranda reflecting on their mentoring experience, it became apparent that the condition of perceived importance was lacking in their relationship. As I noted above, Melissa did indicate that at the onset of the relationship she did perceive it as important; however, this condition is only present if both participants express feeling the relationship and experience was important. Miranda reported that the work she did during the mentorship was not engaging for her and that it did not benefit her career goals.

The only question I ever asked myself is 'Am I too qualified for this internship?' Cause it was very base...they were in the beginning of their research, so it was a lot of data entry, which helps the time go by, but I felt like I didn't get as much...me being where I am in my life and my career, I didn't feel that I got as much out of it as somebody who was, you know, without a career, without the knowledge base would have got. I think I would have chosen a different research project for myself. Only because they were in the early stages, so I didn't get to hone in on my skills - I got to kill time.

At this point what emerges from Melissa and Miranda's interviews is that a number of conditions were never established in their relationship. Miranda's comments demonstrate a lack of perceiving the contact as important and that she was unclear about her mentor's role as well as her own role in the relationship. Additionally, Melissa's responses indicate that the condition of mutual liking was not a feature in their relationship. Idetermined thatequal-status was not a feature of their relationship. Neither Melissa nor Miranda talked about how they worked together. Melissa did indicate that there was the opportunity for Miranda to be a part of the research team and participate in professional conferences, which does seem to set the stage for equal-status to emerge. However, Miranda never confirmed this. In fact, Miranda only referenced data entry and 
looking through journal articles as her research tasks and described the tasks as mundane, which indicates equal-status was not present in the contact.

Kathy and Jarrod's interviews did not provide the same level of detail as the previous pair; however, it was discernible that they too were lacking optimal conditions in their relationship. For example, Jarrod's response to the question "What did you enjoy most about your mentoring experience?" was "I guess getting paid." The follow up question, "What did you get out of your mentoring experience?" elicited the response, “Just knowing that I wouldn't want to be a researcher and have to interview people or do data entry all day." This suggested that he was not engaged in the work nor perceived the experience or relationship as important. Additionally, when Kathy was asked how she contributed to Jarrod's transition from community college to the university and career aspirations, she answered

That's hard to say. I think what Jarrod got out of it was that he doesn't want to do social science research. Um, and I think he was thinking he was going to take more computer classes, and I think maybe it helped him find that direction. I think he is responsible and, you know, he seems very smart and he's a hard worker. Um, I don't know if...I don't think I helped him the transition but the experience might have helped him find direction.

Kathy's response indicated that she did not work with Jarrod to further his academic goals, as she appeared to be unclear about what they even were, and Jarrod's answers suggested he was not engaged with the research. Both Kathy and Jarrod suggested a lack of equal-status, as the mentorship was built around the needs of the research agenda and not promoting Jarrod's academic growth. Thus, I labeled the conditions of cooperating towards a shared goal and equal-status absent in their mentoring relationship. 
Another explanation for why Kathy and Jarrod did not experience mutual liking or perceive their mentoring relationship as important that emerges from both of their interviews is that the two never found any commonalities or common ground. Jarrod explained that "we never talked about our interests." Kathy frequently mentioned that the two did not share any interests with which to build on to form a connection. For example, Kathy said, "Jarrod and I were mismatched in terms of interest and personality, but I don't have any ill will towards him...I don't think it was fun for him; it wasn't fun for me."

To recap, Melissa and Miranda (Pair \#5) were categorized as not meeting the conditions of frequent contact, perceived importance, cooperating towards a shared goal, clearly defined roles, mutual liking, or equal-status. Similarly, Kathy and Jarrod (Pair \#4) were coded as lacking the conditions of perceived importance, mutual liking, cooperation, and equal-status.

\section{The Racial Component}

Both of the white mentors (Kathy and Melissa) who experienced unsuccessful mentoring relationships did not believe that the racial differences between themselves and their protégés had any bearing on the outcome of their relationship. Kathy was adamant that the real reason her and Jarrod did not connect was his disinterest in her research project. When she was asked if there was anything that could have been done differently to encourage a closer bond between a mentor and protégé with different racial backgrounds Kathy responded: 
I mean I don't know, I mean I...I think it depends on the nature of the project too. If you find someone who is interested in your project that you're doing that kind of transcends all those...um, and if not, it doesn't really matter anyway, you know, because they're not interested in the work.

Kathy's response indicated that because Jarrod was not interested in her project it was not important for her to consider how the racial dynamics might affect their interactions, and if he had been interested in the research the racial dynamics would not have influenced their relationship because their common interest would supersede the racial component of their mentorship. Thus, regardless of Jarrod's level of interest in the research Kathy did not perceive race as a factor in their relationship. While Kathy claimed that race was inconsequential in their mentorship, she appeared uncomfortable when answering the questions that explicitly asked about race. Kathy did not give any indication that she was aware of implicit racial attitudes or exhibit any race awareness during her interview.

Similarly, Melissa was not aware of how she and Miranda's racial differences had any significance on their relationship. Melissa was asked if her mentoring experience with Miranda had any impact on how she thought about mentoring a protégé of a different racial or ethnic background in the future. She answered with, "I just don't see how race, ethnicity, class, minority, anything...I just don't see how that came out. I really don't, and I thought about it." While Melissa did not appear uncomfortable during the questions about race and ethnicity, she did not elaborate on these questions like she did with the other questions. There was nothing in Melissa's interview that revealed a high level of race awareness. However, she did mention prior mentoring relationships with minority protégés, but again indicated that race was not a factor and that group saliency was not strong in those relationships, as recalling their ethnicity was difficult for her. It 
was also difficult to gauge how strong those relationships were, because they occurred many years ago.

The protégés (Jarrod and Miranda) differed in their responses about how race might have played out in their mentoring relationships. Jarrod did not elaborate on any of the questions about race. He simply said "No" race did not have any significance on his relationship with Kathy. Miranda, however, did discuss race in more detail.

It makes me wonder how things would have went if we were of the same background. I didn't think anything of it while I was going through the internship, cause I thought it was a job and I early on learned that I would be working with people of different backgrounds... I think we would have...not only if our stories would have been more aligned would I have gained something different, cause feeling that connection I think is important on many levels when we're looking at it as a mentorship because you're trusting in this person. You're trusting in this person to provide you with valuable input for your career, your life.

This response from Miranda echoed a lot of the other protégés responses when asked how their mentoring relationship might have been different if their mentoring relationship had been a same-race mentorship. What is interesting about Miranda's explanation is she seemed to suggest that it is more difficult to establish a connection and trust in an interracial relationship than a same-race relationship.

To summarize, three out of the four respondents (Kathy, Melissa, and Jarrod) did not indicate that any of the conditions related to contemporary racism (race awareness, aware of implicit racial attitudes, belonging to a nonracial out-group, or prior interracial interactions) were present in either mentoring pair. While Miranda did reflect on how the racial differences might have impacted her mentoring relationship, her mentoring 
relationship is still categorized as not involving any of the racial conditions, as the conditions were not expressed in Melissa's interview.

\section{Institutional Support}

All of the participants were asked a series of questions about the structure of the mentoring program, New Horizons. The questions were designed to elicit information about the participants' perceptions on how much support they felt from the program's structure and coordinating staff. In addition to this, the questions were aimed at gauging the participants' feelings on how the program helped them develop a bond with their mentoring partner specifically, and with a person from with a different racial or ethnic background more generally. The participants were then asked what, if anything, they thought the program should do to increase the level of support in developing a successful mentoring relationship. However, upon analyzing these data, I found it difficult to confidently determine if the condition of institutional support was present for any of the participants. Therefore, this section is organized according to the most common ways the participants described the program's level of support and the most frequently mentioned critiques. I begin with a discussion on how the complete pairs described the support they received, followed with how the participants in the supplemental data groups discussed the program.

I begin with the two complete pairs that are categorized with an outcome of a successful interracial mentoring experience. These pairs are Pair \#1 (Karen/mentor and Ryan/protégé) and Pair \#6 (Tom/mentor and Maria/protégé). Out of this group, Tom was the only participant to offer suggestions about what the program could do better. Tom 
said, "More feedback should be provided from the previous years so mentors can know what worked...It seems like they just start all over again every year instead of building on year after year." Karen commented that the program provided a welcoming and diverse environment, which she explained helped protégés and mentors develop a connection. The conversation then turned toward the larger university and how the university itself needs to create a more inclusive and welcoming atmosphere. Both of the protégés (Ryan and Maria) from these two pairs, credited the program for matching them with their mentor, but that developing the connection between protégé and mentor was up to the mentor and protégé. However, Ryan and Maria did not think the program needed to change anything. Thus, Pair \#1 and Pair \#6 are not categorized as having institutional support, but are not labeled as not having it either; in other words, it is unclear if institutional support was present.

For the complete pairs that were labeled with medium success, Pair \#2 (Jill/mentor and Andrea/protégé) and Pair \#3 (Patrick/mentor and Renee/protégé) there was not institutional support. Jill did not think the program was clear enough in their guidelines for the poster presentations the protégés do at the end of the mentorship. She also thought the program should do more for the protégés, in the sense of better socializing the protégés into the academic community. Patrick, Renee, and Andrea all commented that the program did not do anything to help the mentoring pairs develop a better connection. However, Patrick did not think the program should do more. Pair \#2 and Pair \#3 are categorized as not including institutional support. 
The two complete pairs in the low-to-no success group are Pair \#4 (Kathy and Jarrod) and Pair \#5 (Melissa and Miranda). For Pair \#4, neither Kathy nor Jarrod indicated that they felt a high level of institutional support. Kathy suggested that regardless of the level of institutional support, her and Jarrod would not have developed a better connection. Both of them offered the same suggestion for enhancing the program: more input from the mentors and protégés when matching the pairs together. In respect to Melissa and Miranda (Pair \#5) they are categorized as lacking institutional support. Melissa discussed how much potential the program has, but that it does not have the infrastructure it needs to meet those goals. She felt like she was not given clear enough guidelines on how she was supposed to meet the program's goal of helping her protégé transition into the university. In this sense, she explained that too much emphasis is on the research component and not enough on the actual mentoring component. Miranda echoed Melissa's feelings, saying:

Well as long the mentor and the mentee understands that you are here not to just complete a task, but to actually mentor your mentee. And both people are in line with what the end goal is, then maybe it would strengthen that bond or create opportunities for that bonding to happen. But if a mentee goes into it looking at it as, "Okay this an internship. I'm going to get some job experience." And doesn't look at it beyond that. And that's why I say maybe it was my fault by not looking at...because I didn't feel she was a mentor.

Thus, Melissa and Miranda (Pair \#5) are labeled as not having institutional support.

The five protégés (Nathan, Candice, Hector, Alex and Paul) in the supplemental data group all said that the program only helped them connect with their mentor by introducing them to one another, after that they were on their own. Nathan, Candice, and Paul all indicated that that was enough for them and did not think the program should do 
more. However, Alex and Hector both thought the program should do more consistent check-ins with the mentoring pairs so that they can help those that are not connecting. Alex also thought they should incorporate more activities for the mentoring pairs outside of the research tasks to enhance the bonds.

The two mentors (Eddie and Laura) differed slightly on their opinions of the program's support. Laura thought the program did plenty and that she probably did not take advantage of all the resources. She also mentioned that if they added more activities or sessions for the mentors it would be too much of a burden on her time. On the other hand, Eddie thought the program could do more to help facilitate better connections. However, he also recognized how difficult it is for the program to find mentors in the first place so asking them to do more would most likely result in even less faculty participation. Thus, all the participants in the supplemental data group are not coded as having institutional support in their mentorship.

\section{Conclusion}

The mentoring pairs and participants from the supplemental data group described a variety of cross-race mentoring experiences, which illuminated how many of the optimal conditions work together, or do not work together, to influence the outcomes of their mentorship. Those mentoring pairs that exhibited the most conditions described the most successful outcomes. Interestingly, these successful pairs also demonstrated the conditions throughout their discussions in different areas of the relationship. Mentors that perceived the mentoring role as important and understood what the role entails were more

likely to promote their protégés' academic and career aspirations. This most often led the 
protégé to also perceive the relationship as important and they were more engaged in the research tasks they worked on. Additionally, the mentors with the most successful mentoring experiences demonstrated an ability, which is unique among whites in our culture, to acknowledge the barriers students of color often face, particularly within higher education.

The mentoring pairs with a medium success outcome did demonstrate a number of the conditions, but they did not emerge as often as in the successful mentoring relationships and they did not emerge in multiple aspects of their relationship. The level of agreement between mentor and protégé was often lacking among these complete mentoring pairs as well. I could not confidently determine if the conditions related to contemporary racism were present in one pair, Patrick and Renee, because they indicated that Renee identified as (and Patrick perceived Renee as) an immigrant minority and not a racial minority. This pair indicated they perceived the relationship as intercultural rather than interracial.

The cross-race mentoring pairs that described unsuccessful mentoring experiences had two very different experiences, however, they did share one thing in common: they did not confirm the presence of any of the conditions among mentor and protégé in their relationship. For Kathy and Jarrod the lack of perceived importance and a shared interest appeared to be significant factors in why the two never developed a real connection and mutual liking. The mentors in both of these pairs did not demonstrate the presence of any of the conditions related to modern racism (race awareness, membership in a nonracial out-group, aware of implicit racial attitudes, etc.). In fact, Miranda was the only 
participant in this group that considered how race and ethnicity might have impacted the mentoring relationship.

Finally, the condition of institutional support lacked an adequate amount of data that addressed this condition. Only Melissa and Miranda indicated that the condition was lacking; however, there was not a complete pair that demonstrated the condition was present. It was clear that there was not on-going training for the mentors and structured activities for the mentoring pairs were not a part of the program's structure. At the same time, a number of the mentors explained that the program might become a burden if there were required sessions for the pairs to attend, as much of the focus was on the research tasks.

In sum, the data generally supports all three hypotheses: (1) mentors and protégés whoperceive their cross-race mentoring relationships as successful will be those who experiences all, or most, of the conditions in Table 1; (2) protégés and mentors who perceive their cross-race mentoring relationship as somewhat successful will be those who experienced some, but not most, of the conditions in Table 1; and (3) protégés and mentors who perceive their cross-race mentorship as not successful will be those who experienced none, or very few, of the conditions in Table 1. The following chapter will offer a more in-depth discussion about how the data conveyed the interconnectedness of the conditions and what this contributes to our understanding of cross-race mentoring relationships, specifically, and the intergroup contact hypothesis more generally. 


\section{CHAPTER VI: DISCUSSION}

I would like to start by summarizing my study's findings and presenting a table to provide a visual representation of these findings. The first pair (Pair \#1), Karen and Ryan, confirmed that the following conditions were present: frequent contact, perceived importance, cooperation towards a shared goal, equal-status within the relationship, mutual liking, group saliency, aware of implicit racial attitudes, race awareness, and Karen had nonracial out-group experience. The conditions, institutional support and past experience with developing meaningful interracial relationships, and clearly defined roles, could not be confidently determined as present in their relationship. At the same time, these conditions could not be labeled as absent either, as there was not enough information to assess whether they were present or absent. I categorized Karen and Ryan with the outcome of successful. The next pair (Pair \#6), Tom, Julie, and Maria, affirmed the presence of conditions: frequent contact, perceived importance, cooperation, equalstatus, mutual liking, group saliency, aware of implicit racial attitudes, and race awareness. However, Pair \#6 did not confirm the presence of institutional support, past interracial relationships that were meaningful, clearly defined roles, and that the white mentor had experience as a nonracial out-group member. Tom, Julie, and Maria were also labeled with a successful outcome. Both of these mentoring pairs confirmed the presence of at least eight of the twelve optimal conditions (Pair \#1 confirmed nine). However, what is most striking about these pairs is that many of the optimal conditions emerged in multiple aspects of their mentoring relationship and often in conjunction with one another, which is discussed in further detail below. 
The next two mentoring pairs, Pair \#2 and Pair \#3, were both determined to have a medium successful outcome for the cross-race mentoring experience. First, Pair \#2 (Jill and Andrea) indicated that the conditions, perceived importance, mutual liking, race awareness, and Jill's prior experience of developing meaningful cross-race relationships, were somewhat present in their relationship. When the two did indicate these conditions, they were not conveyed in all areas of their relationship or as strongly as the two pairs with a successful outcome. The conditions, frequent contact, equal-status, cooperation towards a shared goal, membership in a nonracial out-group, and aware of implicit racial attitudes, were not present in their relationship. The remaining conditions, institutional support, clearly defined roles, and group saliency, could not be confidently determined as present or absent in their mentoring relationship. The next pair (Pair \#3), Patrick and Renee, did convey the presence of frequent contact and mutual liking; however, the condition of mutual liking was not rooted in a personal connection in the same way that it was for Pair \#1 and Pair \#6. Additionally, the two confirmed that perceived importance, cooperation, and equal-status were present in some aspects of their relationship, but that they were also absent in other aspects of their mentorship. The conditions, aware of implicit racial attitudes, experience developing meaningful cross-race relationships, race awareness, member of a nonracial out-group, and group saliency were not present in their relationship. Institutional support and clearly defined roles could not be determined to be either present or absent in their relationship. In sum, these two mentoring pairs established some of the optimal conditions, but not most of the conditions, and many of the conditions were present in some areas of their relationship but not all aspects of their mentorship. 
Finally, the last two pairs, Pair \#4 and Pair \#5, both experienced unsuccessful cross-race mentoring experiences. Neither pair was able to demonstrate that any of the conditions were present. Melissa, the mentor from Pair \#5, did indicate perceived importance in relation to her role as a mentor; however, because Miranda, the protégé, did not perceive the relationship as important, their mentoring relationship did not establish this condition. Kathy, the mentor from Pair \#4, did describe perceived importance in relation to advancing her own research agenda; however, because the research tasks that were allocated to Jarrod, the protégé, were mundane research tasks (i.e., inputting data), Jarrod did not perceive this as important or contributing to his own academic and career goals, and therefore, the mentoring relationship lacked perceived importance.

In sum, all three hypotheses were supported by my data. Hypothesis \#1 predicted that the mentoring pairs with a successful outcome would have all or most of the optimal conditions present in their relationship. While neither pair with a successful outcome demonstrated the presence of all the conditions, they did indicate at least eight of the twelve conditions. Hypothesis \#2 proposed that the mentoring pairs with an outcome of medium success would have some conditions present, but not all or most of the conditions present. Both pairs with a medium success outcome conveyed the presence of at least four of the twelve conditions; however, these conditions were not present in all aspects of their mentoring relationship. Finally, hypothesis \#3 expected the mentoring relationships with a low-to-no success outcome would not have any or very few of the conditions present. Neither pair with low success indicate that any of the conditions were present in their relationship. In addition to finding support for my hypotheses, I 
discovered that it is not merely the presence of the conditions that explained the success for these cross-race mentoring relationships. After analyzing these data I discerned that the presence of these conditions in multiple aspects of their relationships and the interconnectedness of these conditions offer the best explanation for the outcomes of their experiences.

The impact of institutional support could not be determined from my data. I was not able to confidently categorize any of the pairs as having this condition present; at the same time, Pair \#5 (Melissa and Miranda) was the only pair I confidently determined to not have institutional support. Thus, it is difficult for me to make an analysis on how this condition interacted with the other optimal conditions.

Below, Table 2 provides a visual of these findings. 


\begin{tabular}{|c|c|c|c|c|c|c|}
\hline \multicolumn{7}{|c|}{$\begin{array}{c}\text { TABLE } 2 \\
\text { Conditions Present in Cross-Race Mentoring Relationships }\end{array}$} \\
\hline & $\begin{array}{l}\text { Karen and } \\
\text { Ryan } \\
\# 1\end{array}$ & $\begin{array}{c}\text { Tom, Julie, } \\
\text { and Maria } \\
\quad \# 6\end{array}$ & $\begin{array}{c}\text { Jill and } \\
\text { Andrea } \\
\# 2\end{array}$ & $\begin{array}{c}\text { Patrick } \\
\text { and } \\
\text { Renee } \\
\# 3\end{array}$ & $\begin{array}{c}\text { Kathy } \\
\text { and } \\
\text { Jarrod } \\
\# 4\end{array}$ & $\begin{array}{l}\text { Melissa } \\
\text { and } \\
\text { Miranda } \\
\# 5\end{array}$ \\
\hline \multicolumn{7}{|l|}{ Conditions } \\
\hline 1. Frequent contact & $*$ & $*$ & $\mathbf{X}$ & $*$ & $\mathbf{X}$ & $\mathbf{X}$ \\
\hline $\begin{array}{l}\text { 2. Institutional } \\
\text { Support }\end{array}$ & - & - & - & - & - & $\mathbf{X}$ \\
\hline $\begin{array}{l}\text { 3. Cooperation } \\
\text { towards a shared } \\
\text { goal }\end{array}$ & $*$ & $*$ & $\mathbf{X}$ & $* /-$ & $\mathbf{X}$ & $\mathbf{X}$ \\
\hline 4. Equal-status & $*$ & * & $\mathbf{X}$ & */- & $\mathbf{X}$ & $\mathbf{X}$ \\
\hline 5. Mutual liking & $*$ & $*$ & $* /-$ & $* /-$ & $\mathbf{X}$ & $\mathbf{X}$ \\
\hline 6. Group saliency & * & $*$ & - & $\mathbf{X}$ & $\mathbf{X}$ & $\mathbf{X}$ \\
\hline $\begin{array}{l}\text { 7. Perceived } \\
\text { importance }\end{array}$ & $*$ & $*$ & */- & */- & */- & $* /-$ \\
\hline $\begin{array}{l}\text { 8. Clearly defined } \\
\text { roles }\end{array}$ & - & - & - & - & $\mathbf{X}$ & $\mathbf{X}$ \\
\hline $\begin{array}{l}\text { 9. Aware of implicit } \\
\text { racial attitudes }\end{array}$ & * & $*$ & $\mathbf{X}$ & $\mathbf{X}$ & $\mathbf{X}$ & $\mathbf{X}$ \\
\hline 10. Race awareness & $*$ & $*$ & */- & - & $\mathbf{X}$ & $\mathbf{X}$ \\
\hline $\begin{array}{l}\text { 11. Member of } \\
\text { nonracial out-group }\end{array}$ & $*$ & $\mathbf{X}$ & $\mathbf{X}$ & $\mathbf{X}$ & $\mathbf{X}$ & $\mathbf{X}$ \\
\hline $\begin{array}{l}\text { 12. Experience with } \\
\text { forming interracial } \\
\text { relationships }\end{array}$ & - & - & $* /-$ & - & - & - \\
\hline $\begin{array}{l}\text { Outcome of Cross- } \\
\text { Race Mentoring } \\
\text { Experience }\end{array}$ & Successful & Successful & $\begin{array}{l}\text { Medium } \\
\text { Success }\end{array}$ & $\begin{array}{l}\text { Medium } \\
\text { Success }\end{array}$ & $\begin{array}{l}\text { Low-to- } \\
\text { No } \\
\text { Success }\end{array}$ & $\begin{array}{l}\text { Low-to-No } \\
\text { Success }\end{array}$ \\
\hline \multicolumn{7}{|c|}{$\begin{array}{l}\text { KEY: } \\
*=\text { Condition present for both members of mentoring pair, and the condition was mutually reinforcing with } \\
\text { other conditions } \\
\mathrm{X}=\text { Condition not present } \\
* /-=\text { Condition somewhat present, but not mutually reinforcing with other conditions } \\
\text { _ = Unclear if condition is present or absent }\end{array}$} \\
\hline
\end{tabular}




\section{The Interconnectedness of Optimal Conditions}

This section discusses the conditions that emerged most often and that I determined had the greatest impact on the success (or lack thereof) of these cross-race mentoring experiences. These conditions consist of perceived importance, cooperation towards a shared goal, equal-status within the relationship, and mutual liking. These four conditions manifested in a number of ways according to my data. The most frequent aspects of the mentorships where these conditions emerged were: (1) mentor's motivations and goals for becoming a mentor; (2) protégé's reported engagement in the research tasks; (3) if and how the mentor made an effort to advance the protégé's academic/career aspirations; (4) how each participant described their mentoring partner; and (5) protégé's description of how they benefited from their cross-race mentoring experience. Not only were these conditions discussed in great detail among the most successful mentorships; they also revealed a mutually reinforcing dynamic between the conditions. Therefore, this discussion is arranged as an analysis of how these conditions are linked together.

Interestingly, perceived importance was one of, if not the most, instrumental condition in promoting a successful cross-race mentoring experience. Perceived importance was particularly impactful among the mentors who explained their motivations and goals for mentoring in respect to how mentoring would benefit their protégé and the larger climate in the university. The mentors who perceived the mentoring relationship as important because of the mentoring function also knew the most about their protégé's personal goals and offered multiple examples of how they contributed to their protégé's personal and professional development. This was confirmed 
by the protégés (Ryan and Maria) in two of the six complete pairs (Pair \#1 and Pair \#6).

In contrast, the mentors (Patrick, Jill, and Kathy) did not convey perceived importance in light of their mentoring role; rather, their research agendas emerged as the most important reason for deciding to become a mentor, or as the criteria that determined if the relationship was a success to them. However, out of these three mentors, Patrick was the only one that made an effort to provide his protégé, Renee, with research tasks that were engaging and allowed Renee to step into the role of a lead researcher. Both Patrick and Renee did indicate perceived importance surrounding the research tasks, but not in the area of contributing to Renee's aspirations outside of enhancing her research skills or upcoming transition from Northwest Community College to University of the Pacific Northwest. The mentors (Karen, Julie, and Tom) that perceived the mentoring role as important, and therefore, made an effort to promote the protégés' personal goals were at the same time promoting the condition, cooperation towards a shared goal. This suggests support for Van Dick et al. (2004) who found perceived importance to be a mediating condition in intergroup contact that reduced out-group prejudice ${ }^{11}$. They determined that perceived importance emerges when an individual perceives the contact as promoting a personal goal (Van Dick et al. 2004). This explains why the complete mentoring pairs with mentors (Jill, Patrick, and Kathy) who perceived the mentoring relationship as a mechanism to advance their own research agenda, but not as a vehicle to enhance the student protégés' academic and career goals, were not able to corroborate the presence of perceived importance among protégé and mentor in all aspects of their relationship.

\footnotetext{
${ }^{11}$ I use the word suggests and not offers because this study could not determine if out-group prejudice was reduced because of this mentoring experience, as all of the data was collected after the mentoring experience.
} 
The condition of cooperation towards a shared goal frequently emerged alongside the condition of perceived importance. This is not surprising given that individuals perceive intergroup contact situations as important when they feel it is contributing to a personal goal (Van Dick et al. 2004). While this does suggest a very close link between these two conditions, the conditions are still distinct from one another. What emerged from my data is that perceived importance for the mentor's role as a mentor was a catalyst for the condition of cooperation to be established; however this was not the case when perceived importance arose only in respect to the mentor's research agenda. The one exception was Patrick and Renee (Pair \#3), which I will discuss further below. The mentors who understood the importance of their role as a mentor established cooperation towards a shared goal by making efforts to promote the protégés' personal academic and career goals; this in turn resulted in the protégés perceiving this relationship as important. For example, the three mentors, Karen, Julie and Tom, described creating opportunities for their protégés that were outside of the research tasks and specific to the protégés' academic goals. The condition of cooperation also emerged in three of the six pairs (Pair \#1, Pair \#6, and Pair \#3) in the way the mentor and protégé described the research tasks they worked on together. It was clear that cooperation was present when the protégé was engaged in the research work and felt like they were making a significant contribution to the research. This condition was very closely linked to perceived importance. For example, when the student protégé was engaged in the research tasks and encouraged to significantly contribute to the research, they (Ryan, Maria, Renee, Alex, Paul, Candice, and Nathan ${ }^{12}$ ) all described feeling more confident in their educational capabilities and

${ }^{12}$ Alex, Paul, and Candice were in the successful supplemental data group; Nathan was in the medium 
motivated to pursue careers in research. Thus, the research task became a professional goal for the protégés, and was not perceived as just a job, except in Renee's case. While Renee did mention being more confident in her research skills, she still thought of this mentoring experience as a job. Similarly, when the mentor presented their protégé with engaging research work the mentor was involved in a collaborative dynamic with their protégé. The collaborative nature also encouraged the condition of equal-status within the relationship; however this was not the case for the protégés (Andrea, Jarrod, and Miranda) who were assigned the mundane research tasks such as inputting data, organizing bibliographies, or photo-copying articles.

The condition of equal-status was a difficult dynamic to establish within these cross-race mentoring pairs with white faculty members and student protégés of color. However, when mentors perceived their mentoring role as important (Karen, Tom, Julie, Eddie, and Laura ${ }^{13}$ ) and put equal emphasis on promoting their own research agenda goals and their protégés' academic and career goals, equal-status did emerge. There was one mentoring pair, Pair \#3, (Patrick and Renee) that indicated the condition of equalstatus was somewhat present, but not in all areas of their mentoring relationship. For example, Patrick (mentor) did engage Renee (protégé) in research tasks that allowed her to step into his role as a lead researcher and he encouraged Renee to contribute her own analysis and understandings to the research. However, there was not equal-status in relation to whose goals were being advanced in their relationship. Neither Patrick nor

success supplemental data group; Ryan and Maria were in the successful complete pairs, \#1 and \#6; and Renee was in the medium success complete Pair \#3.

${ }^{13}$ The condition of equal-status could not be confirmed with mentors Eddie and Laura as their protégés did not participate in this research. 
Renee described efforts on Patrick's part to learn or contribute to Renee's personal academic and career goals. However, only when both members of the mentorship exhibited perceived importance for the mentoring function of the relationship and cooperation towards a shared goal involved both a personal goal of the mentor and protégé, the condition of equal-status emerged in conjunction with the former conditions. These findings support Pettigrew and Tropp's (2006) assertion that the optimal conditions for intergroup contact are most effective when they work together as a package.

In every case that the conditions of perceived importance, cooperation, and equalstatus were present in more than one aspect of the relationship, mutual liking also emerged. Of the complete mentoring pairs, Pair \#1 (Karen and Ryan) and Pair \#6 (Tom, Julie and Maria) expressed the highest degree of mutual liking and demonstrated the pairs had developed a real personal connection. In contrast, Pair \#4 (Kathy and Jarrod) and Pair \#5 (Melissa and Miranda) conveyed the absence of mutual liking and neither the mentors nor the protégés knew much about their mentoring partner outside of their research interests or lack of research interests. While Pair \#2 (Jill and Andrea) and Pair \#3 (Patrick and Renee) did not express a dislike for their mentoring partner, it was clear that the mutual liking that they did exhibit was not rooted in a personal connection.In fact, the mentors from these pairs, Jill and Patrick, often described their protégés, Andrea and Renee, only in terms of their research skills and how they contributed to Jill and Patrick's research agendas. Thus, the condition of mutual liking emerged when the participants not only described one another favorably, but also indicated they had developed a real relationship that was anchored by trust and authenticity, which are also fundamental 
characteristics of effective mentoring relationships (Rhodes et al. 2002; Spencer 2006). Without the conditions of perceived importance, cooperation towards a shared goal, and equal-status there was less motivation for the mentoring pairs to engage in personal sharing and the mutual liking that was expressed was not rooted in a real and personal connection between the two. My data point to the likelihood that the condition of mutual liking is promoted when the conditions perceived importance, cooperation, and equalstatus work in conjunction with one another. This does not contradict Pettigrew and Tropp's (2006) assertion that mutual liking is a condition for intergroup contact that reduces out-group prejudice, as my data was not capable of determining reductions in prejudice. Instead, this highlights the possibility of how the optimal conditions progress and which conditions engender other optimal conditions.

To recap, the optimal conditions perceived importance, cooperation towards a shared goal, and equal-status within the relationship were most effective when present together for promoting the condition of mutual liking. The most successful complete interracial mentoring pairs (Pair \#1 and Pair \#6) often demonstrated these conditions in concert with one another, while the medium success complete pairs (Pair \#2 and Pair \#3) conveyed the presence of these conditions in only some aspects of their mentoring relationship and often described the optimal conditions singularly, indicating that the conditions were not working together to strengthen and reinforce one another. The unsuccessful mentoring pairs (Pair \#4 and Pair \#5) lacked the optimal conditions in almost all aspects of their mentorship. Thus, my data supports the assertion that these optimal conditions are best understood as a collection of conditions that function together (Allport 1954; Pettigrew and Tropp 2006). Additionally, these findings support Van 
Dick's et al. (2004) addition to the ICH, perceived importance, but also indicate that perceived importance is equally important to be present for the in-group and out-group member in the contact and that it is most effective when it emerges in multiple aspects of the relationship.

I now turn the discussion towards the optimal conditions of: group saliency, aware of implicit racial attitudes, race awareness, membership in a nonracial out-group, and past experience forming meaningful cross-race relationships. According to these data, group saliency was closely connected to the group of conditions I proposed from my earlier discussion on contemporary racism. I first summarize what conditions emerged (and which were absent) in the data and follow up with a discussion on how these conditions were connected to one another. Most of the focus here is on the mentors, as they were the in-group members in this contact situation; however, there were protégés that revealed an awareness of implicit racial attitudes in respect to their white mentors' racial attitudes that merits discussion as well.

There were only three mentors, Karen, Tom, and Jill that demonstrated race awareness; however, each of these mentors conveyed different levels of race awareness. Karen indicated the highest level of race awareness, which was accompanied with four additional conditions: awareness of implicit racial attitudes, group saliency, experiences as a member of a nonracial out-group, and perceived importance. Karen was the only participant to mention experiences as a member of a nonracial out-group, and she mentioned it multiple times. Tom's race awareness did not appear as high as Karen's but did emerge in conjunction with the conditions of group saliency and aware of implicit 
racial attitudes. In contrast, Jill exhibited race awareness, but I did not consider it a high level of race awareness; when she did indicate race awareness it was in the context of how other white faculty members lack it or it was in relation to the minority staff member she worked closely with, Rick. Thus, Jill never mentioned race awareness in regards to her mentoring relationship with Maria. Additionally, when she exhibited race awareness it often appeared to emphasize that her own race awareness was greater than most whites in the university and surrounding area. Thus, she also indicated that she did not consider her own implicit racial attitudes as a factor in her interracial relationships. The condition of group saliency arose in a similar manner for Jill; she did not indicate that Maria's (protégé) Latina background was salient to her other than it presented an opportunity for her Latino colleague, Rick, to not feel so racially isolated in their department. The remaining four mentors, Julie, Patrick, Melissa, and Kathy did not indicate any of these conditions during their interviews. However, Julie was the only mentor that upon reflecting on it, remarked that maybe she should have considered the racial and ethnic barriers minority students confront.

Karen and Tom provided the most evidence for how these conditions work together. In most instances when they demonstrated one of these conditions it was done in conjunction with other conditions. For example, when Karen reflected on her experiences as a woman grad student in what was essentially an all male university she provided an example of how she felt when the male students assumed she was a teacher because of their implicit gender attitudes. This was done in the context of describing how her black protégé, Ryan, must feel when he walks into a room full of white people and he is the only black person. When Tom discussed the impacts of race and ethnicity, he too, 
exhibited more than one of these conditions. Furthermore, Karen and Tom were the only mentors from the complete pairs that acknowledged how their mentoring relationship would differ if it were a same-race match. It was clear from Karen and Tom that because they were racially aware, they were more cognizant of the barriers faced by students of color and considered these factors when beginning to develop a relationship with their protégé. It appears that when these conditions, race awareness, aware of implicit racial attitudes, group saliency, and experiences as a nonracial out-group member make the biggest contribution to positive interracial relationships when they are present alongside one another.

While I cannot confidently say that the mentors who did not demonstrate race awareness necessarily lack race awareness, these mentors often avoided the questions that were explicitly about race and ethnicity or quickly changed the direction of the conversation away from race and ethnicity. I can say with confidence that these mentors did not have a high level of race awareness, as they all claimed that their racial difference with their protégé played no role in their relationship. My data support Trepagnier's (2010) assertion that whites with low levels of race awareness often avoid discussing racial topics.

Interestingly, the protégé participants were much more likely to discuss the racial differences between themselves and their mentors. At the same time, the protégés indicated that these differences had the most impact at the very beginning of their mentoring relationships. In fact, every protégé remarked that by the end of the mentorship race and ethnicity did not hold much influence on how they perceived or felt 
about their mentor. However, out of the eleven protégé participants, seven felt they would have felt more comfortable with a same-race mentor at the onset of their relationship. From this group, four believed they would have developed a closer relationship with their mentor had they shared the same racial or ethnic background. Ryan and Maria, the two protégés with the mentors who demonstrated the most optimal conditions in relation to contemporary racism ${ }^{14}$, did not believe they would have gained more from having a same-race mentor. Therefore, I discerned that white mentors who can acknowledge and overcome the pitfalls of implicit racial attitudes are better equipped to foster successful interracial mentoring relationships.

I now turn my attention to the two conditions not yet mentioned in this discussion: frequent contact and institutional support. First, frequent contact was most significant when it was present alongside the additional conditions. Both successful cross-race mentoring pairs (Pair \#1 and Pair \#6) did establish frequent contact. Within the medium success interracial mentorships there was one pair (Pair \#3) that did establish frequent contact and one (Pair \#2) that did not. Neither pair (Pair \#4 and Pair \#5) indicated frequent contact. Again, this implies that frequent contact is most effective as an optimal condition when present in combination with the other conditions.

My data support Pettigrew and Tropp (2006) who found that the optimal conditions are most effective when present together and working in conjunction with one another. The impact of perceived importance appeared to play a pivotal role in fostering many of the other conditions as well, which lends support to Van Dick et al.'s (2004)

\footnotetext{
${ }^{14}$ The conditions of: race awareness, group saliency, aware of implicit racial attitudes, experience with building meaningful cross-race relationships, and experiences with being a member of a nonracial outgroup.
} 
discovery of this relationship and its significance. My findings also highlight that perceived importance only occurs as a significant condition if it is present from both sides of the relationship. Additionally, the data indicate that the white mentors who were able to overcome the common pitfalls of contemporary racism, and thus demonstrated race awareness and recognized the impacts of implicit racial attitudes, were also the white mentors that exhibited empathy for their protégé and were cognizant of the barriers racial minorities confront in institutions of higher education.

Following the data analysis process, it became evident that delineating between each distinct condition is not always a clear cut process. At times the specific characteristics of each condition blended in such a way that they became less distinct, particularly in the cases of the most successful cross-race mentoring experiences. For example, it was often difficult to determine if the participants were describing the condition of equal-status or cooperation towards a shared goal, particularly in the cases of the most successful interracial mentoring experiences. Equal-status emerged when the mentor and protégé both explained feeling like their own goals were being advanced because of their mentoring relationship. For the mentors this was often discussed in relation to their research agenda; for the protégés it was often explained in relation to their academic or career aspirations being moved forward. Thus, when both mentor and protégé worked to advance one another's goals, the two were also cooperating together towards a shared goal. The methodological question can be reasonably raised, are these two distinct "variables"? I believe they are two distinct conditions, but can at times manifest in similar forms. Although there was overlap between the two conditions at times, this was not always the case. The ovelap demonstrates how the conditions 
reinforce one another, rather than indicating that the conditions are not distinct from one another. Another example of conditions that appeared to bleed together involves race awareness and awareness of implicit racial attitudes. It is not surprising that the conditions of race awareness and aware of implicit racial attitudes often arose without a clear distinction from one another. Indeed, when Trepagnier (2010) explicates the components of race awareness, being cognizant of one's own racial assumptions and understandings is a critical component for developing a higher level of race awareness. This is very closely tied to Dovidio et al.'s (2002) research on implicit racial attitudes. Although Trepagnier does not use the language of implicit and explicit racial attitudes, she does explain that whites informally learn negative information about nonwhites, which is often internalized and becomes the foundation for interpreting the actions and behaviors of nonwhites. Here again, the methodological question can be raised, are these two really distinct variables? I belive they too are distinct from one another, but involve a great deal of overlap. My data suggests that a distinction needs to be made between whites who understand the continuing impacts of race and racism in the United States (i.e. race awareness) and whites who question their own racial attitudes and assumptions. While I did not discover any participants that expressed their awareness of implicit racial attitudes without also exhibiting race awareness, I did find participants that demonstrated race awareness but never provided any indication of an awareness of their own implicit raical attitudes.

\section{Implications}

This part of the discussion addresses the implications and contributions of my data for both (1) interracial mentoring, and (2) intergroup contact theory. As the 
mentoring literature to date remains underdeveloped in the area of interracial mentoring, specifically in the context of white adult mentors and young minority protégés, I will begin my discussion here. I then identify this study's contributions to the diverse literature on the intergroup contact theory.

The literature on cross-race mentoring places much of its focus on determining if cross-race matching yields the same benefits for protégés as same-race matching (DuBois et al.2002; Campbell and Campbell 2007; Santos and Reigadas 2002). While this is certainly an important question to examine, it does little to broaden our understanding of how cross-race mentoring relationships develop or how contemporary U.S. racism might impede these relationships. Researchers discovered that cross-race mentoring can, and often will, benefit protégés of color (DuBois et al. 2002; Campbell and Campbell 2007). In fact, DuBois et al. (2002) found that race and ethnicity are not factors that can account for why a mentoring relationship produces the desired benefits or does not; instead, it is the mentoring programs' procedures and practices that produce the greatest impact on the outcomes of mentoring interventions. My findings suggest that disregarding the role of race and ethnicity altogether is not advisable, particularly when considering the start of the relationship. Despite this, these data do not necessarily contradict DuBois' et al. (2002) argument that the structure of the mentoring program is instrumental in explaining outcomes of mentoring experiences. It could be that cross-race mentoring interventions are even more depended on a well developed infrastructure from mentoring programs to cultivate a strong interpersonal bond between mentor and protégé. However, because my data did not provide adequate information to validly make this assertion more research in this area is needed. Many of the protégé participants described their mentoring 
relationship as having a professional boss/employee dynamic and not a close supportive bond. It could be that a mentoring program's structure is even more critical in the case of cross-race mentoring. Additionally, my study suggests that the mentors' motivations and expectations greatly impact how the relationship develops between the mentoring pair. This supports Grossman and Rhodes (2002) argument that mentors who take on the role to contribute to a more inclusive community are more likely to develop trusting and supportive bonds.

In light of this study's contribution to the body of research on the intergroup contact theory, these findings offer additional confirmation to the impact the optimal conditions have when they are present together and work to reinforce one another (Allport 1954; Pettigrew and Tropp 2006). In addition to this, these data highlight the different aspects of the cross-race mentoring relationships where the conditions were working in concert the most. For example, when the mentor made an effort, because it was important to them, to promote their protégé's academic and career goals the protégé perceived this relationship as important as well. This led to the pair cooperating together to find ways to advance these goals.

Perceived importance emerged as a significant factor for explaining the most successful pairs' outcome. Thus, offering support to Van Dick's et al. (2004) proposal of adding this condition to the intergroup contact theory. However, my data revealed that this condition can manifest in different ways and the outcomes can be completely different depending on where the perceived importance is directed. Perceived importance was most significant for explaining successful outcomes when it led to the pair 
cooperating with one another. Furthermore, when perceived importance was only present for one member of the pair, and not both, it lost its significance. This suggests that perceived importance is not simply a condition that needs to be established for the ingroup member, but that this condition could be equally important in regards to the outgroup member.

In addition to this, these data also point to adding conditions to the ICH that consider recent developments in the area of contemporary US racism. The most successful cross-race mentoring pairs included mentors who exhibited the ability as a white individual to recognize and confront the perils of contemporary US racism. Finally, my data indicate that the optimal conditions are best understood when both the in-group and out-group members' understandings and perceptions are considered. For example, if I had only interviewed the white mentors it is very possible that I would have determined that some conditions were present in the relationship that upon examining the data from their protégé indicates the condition was not present.

\section{Limitations of Study}

A limitation of this study involves the duration of the mentorships that were examined. These mentoring relationships were structured to be a summer long mentorship of only three months. Therefore, it is important to point out that these results might be different if the mentoring relationships were designed to last longer, and the participants had more time to develop a bond with one another. It is likely that a number of these mentoring relationships would have conveyed much stronger connections had they spent more than a summer together as mentor and protégé. At the same time, these 
mentoring relationships shed light on the process of beginning a cross-race relationship and what some of the common perceptions, assumptions, and expectations are for mentors and protégés in higher education interracial mentoring relationships.

\section{Future Research}

The scope of this research examined cross-race mentoring pairs in one formal mentoring program that served first-generation students from underrepresented groups that were making the transition from Northwest Community College to University of the Pacific Northwest. Future research might further build on this research by incorporating multiple mentoring programs in order to compare the different institutional settings. This could shed light on how the structure of the program facilitates the development of the bond between mentor and protégé. Cross-race mentoring relationships that develop informally are another area of interest that can broaden our understanding of these relationships. Comparing how the mentoring relationships develop among formal and informal cross-race pairs can provide insight into what the most important factors are for creating supportive and effective mentoring interventions. In addition, because this research only interviewed cross-race mentoring pairs, future research is needed that compares the understandings and perceptions of those in same-race and cross-race mentoring relationships within the same mentoring program.

Additionally, future researchers interested in intergroup contact can further validate my findings by using the model I proposed and applying it to alternative crossrace situations, other than in the context of mentoring. Examining these optimal conditions in cross-race relationships that are not also bogged down with such drastic 
status differences as education and age as well as the racial and ethnic differences can help refine and affirm the significance of these conditions during interracial contact. 


\section{REFEERENCES}

Alexander, Michelle. (2010). The New Jim Crow: Mass Incarceration in the Age of Colorblindness. New York: The New Press.

Alfaro, Edna C., Adriana J. Umaña-Taylor, Melinda A. Gonzales-Backen, Mayra Y. Bámaca, and Katharine H. Zeiders. 2009. “Latino Adolescents' Academic Success: The Role of Discrimination, Academic Motivation, and Gender.” Journal of Adolescence. 32:941-962.

Allport, Gordon W. 1979(1954).The Nature of Prejudice: Unabridged. $25^{\text {th }}$ ed. Cambridge, MA: Perseus Books.

Anderson, Eugene M., and Anne Lucasse Shannon. 1988. “Toward a Conceptualization of Mentoring." Journal of Teacher Education. 39: 38-42.

Beach, Dennis and Ove Sernhede. 2011. "From Learning to Labour to Learning for Marginality: School Segregation and Marginalization in Swedish Suburbs."British Journal of Sociology of Education. 32(2):257-274.

Berends, Mark and Roberto Peñaloza. 2010. "Increasing Racial Isolation and Test Score Gaps in Mathematics: A 30-Year Perspective." Teachers College Record. 112(4):978-1007.

Bobo, Lawerance D. 2001. "Racial Attitudes and Relations at the Close of the Twentieth Century." Pp. 264-301 in America Becoming: Racial Trends and Their Consequences, Volume 1, edited by N. J. Smelser, W. J. Wilson and F. Mitchell. Washington, DC: National Research Council. 
Bonilla-Silva, Eduardo. 2003. Racism Without Racists: Color-Blind Racism and the Persistence of Racial Inequality in the United States. Lanham, ML: Rowman\& Littlefield Publishers Inc.

Bonilla-Silva, Eduardo and David G. Embrick. 2007. “"Every Place Has a Ghetto...’: The Significance of Whites' Social and Residential Segregation.” Symbolic Interaction. 30(3):323-345.

Burke, Ronald J. 1984. "Mentors in Organizations.” Group \& Organization Management. 9(3):353-372.

Braddock II, Jomills Henry, and Amaryllis Del Carmen Gonzalez. 2010. "Social Isolation and Social Cohesion: The Effects of K-12 Neighborhood and School Segregation on Intergroup Orientations.” Teachers College Record. 112(6):1631-1653.

Campbell, Toni A, and David E. Campbell. 2007. “Outcomes of Mentoring At-risk College Students: Gender and Ethnic Matching Effects.” Mentoring and Tutoring. 15(2):135-148.

Chao, Georgia T., Pat M. Walz, and Philip D. Gardner. 1992. "Formal and Informal Mentorships: A Comparison on Mentoring Functions and Contrast with Nonmentored Counterparts." Personnel Psychology. 45:619-636.

Charmez, Kathy. 2006. Constructing Grounded Theory: A Practical Guide Through Qualitative Analysis. Thousand Oaks, CA: Sage Publications Inc. 
Deutsch, Nancy L., and Jeffery N. Jones. 2008. "'Show Me an Ounce of Respect' Respect and Authority in Adult-Youth Relationships in After-School Programs." Journal of Adolescent Research. 23(6):667-688.

Diversi, Marcelo, and Connie Mecham. 2005. "Latino(a) Students and Caucasian Mentors in a Rural After-School Program: Towards Empowering Adult-Youth Relationships." Journal of Community Psychology. 33(1):31-40.

Dovidio, John F., Samuel L. Gaertner, and Kerry Kawakami. 2002. "Implicit and Explicit Prejudice and Interracial Interaction." Journal of Personality and Social Psychology. 82(1):62-68.

Dovidio, John F., Samuel Gaertner, Kerry Kawakami, and Gordon Hodson. 2002. "Why Can't We Just Get Along? Interpersonal Biases and Interracial Distrust." Cultural Diversity and Ethnic Minority Psychology 8(2): 88-102.

Downey, Douglas B., James W. Ainsworth, and Zhenchao Qian. 2009. "Rethinking the Attitude-Achievement Paradox Among Blacks." Sociology of Education. 82(1):119.

DuBois, David L., Bruce E. Holloway, Jeffrey C. Valentine, and Harris Cooper. 2002. "Effectiveness of Mentoring Programs for Youth: A Meta-Analytic Review." American Journal of Community Psychology. 30(2):157-197.

Eby, Lillian T., Tammy D. Allen, Sarah C. Evans, Thomas Ng, and David L. DuBois. 2008. "Does Mentoring Matter? A Multidisciplinary Meta-analysis Comparing 
Mentored and Non-mentored Individuals.” Journal of Vocational Behavior. 72:254-267.

Ensher, Ellen A. and Susan E. Murphy. 1997. "Effects of Race, Gender, Perceived Similarity and Contact on Mentor Relationships." Journal of Vocational Behavior 50:460-481

Erickson, Lance D., Steve McDonald, and Glen H. Elder. 2009. “Informal Mentors and Education: Complementary or Compensatory Resources?" Sociology of Education. 82:344-367.

Feagin, Joe R. 2001. Racist America: Roots, Current Realities, \& Future Reparations. New York: Routledge.

Gilligan, C., Spencer, R., Weinberg, M.K., and Bertsch, T. 2003. “On the Listening Guide: A Voice-Centered Relational Method.” Pp.157-172 in Qualitative Research in Psychology: Expanding Perspectives in Methodology and Design, edit by P.M. Camic, J.E. Rhodes, and L. Yardley. Washington, DC: American Psychological Association.

Goldsmith, Pat António. 2004. 'Schools’ Racial Mix, Students' Optimism, and the Black-White and Latino-White Achievement Gaps.” Sociology of Education. $77: 121-147$

Grossman, Jean B. and Jean E. Rhodes. 2002. "The Test of Time: Predictors and Effects of Duration in Youth Mentoring Relationships." American Journal of Community Psychology. 30(2): 199-219. 
Hansman, Catherine A. 2002. "Diversity and power in mentoring relationships." In Critical perspectives on mentoring: Trends and issues, edited by C.A. Hansman. Columbus, OH: ERIC

Hewstone, M, and Brown, R. 1986. "Contact is not Enough: An Intergroup Perspective on the "Contact Hypothesis." Pp. 1-44 in Contact and Conflict in Intergroup Encounters, edit by M. Hewstone and R. Brown. Oxford, England: Basil Blackwell.

Hewstone, Miles and Herman Swart. 2011. "Fifty-Odd Years of Inter-Group Contact: From Hypothesis to Integrated Theory.” British Journal of Social Psychology 50:374-386.

Jacobi, Maryann. 1991. "Mentoring and Undergraduate Academic Success: A Literature Review." Review of Educational Research 61:505-532.

Jensen, Robert. 2005. The Heart of Whiteness: Confronting Race, Racism, and White Privilege. San Francisco, CA: City Lights.

Johnson, Allan G. 2000. The Blackwell Dictionary of Sociology: A User's Guide to Sociological Language, Second Edition. Malden, MA: Blackwell Publishers.

Kram, Kathy E. 1983. "Phases of the Mentor Relationship.” Academy of Management Journal. 26:608-625.

Marshall, Catherine and Gretchen B. Rossman. 2011. Designing Qualitative Research: Fifth Edition. Thousand Oaks, CA: Sage Publications Inc. 
Massey, Douglas and Nancy A. Denton. 1993. American Apartheid: Segregation and the Making of the Underclass. Cambridge, MA: Harvard University Press.

Massey, Douglas S. 2004. "Segregation and Stratification: A Biosocial Perspective." DuBois Review. 1(1):7-25.

Pettigrew, Thomas F. and Linda R. Tropp. . 2006. “A Meta-Analytic Test of Intergroup Contact Theory." Journal of Persoanlity and Social Psychology. 90(5):751-783.

Portes, Alejandro and Min Zhou. 1993. "The New Second Generation: Segmented Assimilation and Its Variants." Annals of the American Academy of Political and Social Science. 530:74-96.

Portes, Pedro R. 1999. "Social and Psychological Factors in the Academic Achievement of Children of Immigrants: A Cultural History Puzzle.” American Educational Research Journal. 36(3):489-507.

Reichert, Michael C., Brett Stoudt, and Peter Kuriloff. 2006. “Don’t Love No Fight: Healing and Identity Among Urban Youth.” The Urban Review. 38(3):187-209.

Rhodes, Jean E. 2002. Stand By Me: The Risks and Rewards of Mentoring Today's Youth. Cambridge, MA: Harvard University Press.

Rhodes, Jean E., Ranjini Reddy, Jean B. Grossman, and Judy Maxine Lee. 2002. "Volunteer Mentoring Relationships with Minority Youth: An Analysis of Same Versus Cross-Race Matches." Journal of Applied Social Psychology. 32(10):2114-2133. 
Rhodes, Jean E. 2004. "The Critical Ingredient: Caring Youth-Staff Relationships in After-School Settings." New Directions for Youth Development. 101: 145-161.

Rhodes, Jean E., Ren $\square$ e Spencer, Thomas E. Keller, Belle Liang, and Gil Noam. 2006. "A Model for the Influence of Mentoring Relationships on Youth Development." Journal of Community Psychology. 34(6):691-707.

Riegle-Crumb, Catherine, and Rebecca M. Callahan. 2009. "Exploring the Academic Benefits of Friendship Ties for Latino Boys and Girls.” Social Science Quarterly. 90(3):611-631.

Robinson, Tracy and Janie Victoria Ward. 1991. "'A Belief in Self Far Greater Than Anyone's Disbelief': Cultivating Resistance Among African American Female Adolescents." Pp. 87-103 in Women, Girls \& Psychotherapy: Reframing Resistance, edited by C. Gilligan, A. Rogers, and D. Tolman. New York: Haworth.

Ross-Thomas, Elaine and Charles E. Bryant. 1994. "Mentoring in Higher Education: A Descriptive Case Study.” Education. 115(1):70-76.

Santos, Silvia J. and Elena T. Reigadas. 2002. "Latinos in Higher Education: An Evaluation of a University Faculty Mentoring Program." Journal of Hispanic Higher Education. 1(1): 40-50.

Sellers, Robert M., Nikeea Copeland-Linder, Pamela P. Martin, and R. L'Heureux Lewis. 2006. "Racial Identity Matters: The Relationship between Racial Discrimination 
and Psychological Functioning in African American Adolescents.” Journal of Research on Adolescence.16(2):187-216.

Spencer, Ren $\square$ e. 2006. "Understanding the Mentoring Process between Adolescents and Adults." Youth \& Society. 37(3):287-315.

Trepagnier, Barbara. 2001. "Deconstructing Categories: The Exposure of Silent Racism." Symbolic Interaction. 24(2):141-163.

Trepagnier, Barbara. 2010. Silent Racism: How Well-Meaning White People Perpetuate the Racial Divide. Boulder, CO: Paradigm Publishers.

Van Oudenhoven, Jan P., Jan T. Groenewoud, and Miles Hewstone. 1996. “Cooperation, Ethnic Salience and Generalization of Interethnic Attitudes.” European Journal of Social Psychology 26: 649-661.

Van Dick, Rolf, Thomas F. Pettigrew, Carina Wolf, Vanessa Smith Castro, Ulrich Wagner, Oliver Christ, Thomas Petzel, and James S. Jackson. 2004. "Role of Perceived Importance in Intergroup Contact.” Journal of Personality and Social Psychology. 87(2):211-227.

Wilson, Pamela P., Deborah Valentine, and Angela Pereira. 2002. "Perceptions of New Social Work Faculty about Mentoring Experiences.” Journal of Social Work Education. 38(2):317-332.

Zamudio, Margaret M., and Francisco Rios. 2006. "From Traditional to Liberal Racism: Living Racism in the Everyday." Sociological Perspectives. 49(4):483-501. 


\section{Appendix A: Invatation Letter for Protégés from New Horizons Mentoring Program}

December 1, 2011

Dear $<$ New Horizons Protégé name>:

Hello from the crew at New Horizons, Dr. Cruiz, Vanessa, and Heather. We are hoping this Fall term has been a good one. We are writing to give you a "heads up" that we need you for a follow up interview/chat, and we will be contacting you to set it up for a convenient time between the week after finals and the first couple of weeks in January.One of the many interesting things about our North Pacific University New Horizons Program is that many of the faculty-student pairs match people from different cultural backgrounds. We have decided that it would be a real neat thing to do a follow up interview to collect your story about this experience of having a mentor from a different background. Dr. Eduardo Ramirez, from the Sociology Department, who is one of our New Horizons mentors, is joining us with some of his student, to collect your stories. Please cooperate with Dr. Ramirez and his crew when they call you to set up an appointment. The chat will take an hour, give or take. And, as a token of thanks, you will get a $\$ 15$ certificate.

If you have any questions, please feel free to contact Vanessa (Vjackson@NPU), or Heather (hwilson@ npu.edu), your caring New Horizons leaders, or our director Dr. Cruiz ( $\underline{\text { acruiz@ @ npu.edu }), ~ o r ~ o u r ~ f a c u l t y ~ m e n t o r ~ i n ~ c h a r g e ~ o f ~ t h e s e ~ i n t e r v i e w s, ~ D r . ~ R a m i r e z ~}$ (eramirez@npu.edu).

From all of us at New Horizons, all the best wishes for the end of the term, and for a peaceful and fun holiday break!
Alberto Cruiz
Vanessa Jackson
Heather Wilson 


\title{
Appendix B: Voluntary and Informed Consent for Protégé Participants
}

\author{
BE PART OF AN INNOVATIVE AND IMPORTANT PROJECT
}

\author{
A Master's Thesis from the Sociology Department of North Pacific University
}

\section{Consent Form}

The Sociology Department at North Pacific University is conducting a research study about cross-race youth mentoring pairs. Specifically, this research is focusing on the experiences and perceptions of both participants and identifying practices which facilitate integration.

\section{What Will I Have To Do?}

If you are willing to participate in this research, we will ask you to talk with us for an interview lasting approximately 60 minutes. Some of the topics up for discussion will be:

- Your experience of participating in a cross-race mentoring pair.

- Your thoughts on how being involved in mentoring has impacted your life.

- Your description of the activities you have done with your mentor/mentee.

\section{Are There Any Risks?}

Your mentoring relationship is an important and personal part of your life, and we respect and understand that. You are not required to participate, and nobody will be upset with you if you do not want to. If you do decide to participate, you could feel uncomfortable, sad, confused, guilty, angry, or embarrassed from some of the interview questions asked. You do not have to answer anything you do not want to. At any point, you may stop, and nobody will be upset, then you can continue or stop altogether and that is okay. If you find yourself upset after the interview and want to talk with somebody, you can call Eduardo Ramirez with the NPU Center of Mentoring Research and Sociology Department at 503-724-6504; he is the faculty member leading the project.

\section{What Will I Get In Return?}

- A $\$ 15$ gift card.You will receive the gift card as soon as the interview is over, regardless of completion. This is our way to show our appreciation; we know your time is valuable.

- A chance to share your story and strengthen your mentoring relationship.Discussing the nature of your mentoring relationship has the potential to show its importance and could indicate types of activities that bring the two of you closer.

- An opportunity to help others.Lots of people get pleasure out of helping others. Your story is important and we can learn a lot from you, and teach others how to improve their mentoring relationship. This study also has the potential to contribute to providing adult mentors to minority youth who need them.

\section{What Are You Doing To Protect Me?}


Protecting your identity is extremely important to us and we have taken the following measures to ensure confidentiality.

- We will not tell a single person if you decide to participate or if you decide not to.

- You will be interviewed alone and what you say will be kept private.

- Your name and what you tell us in the interview will be kept private to the extent allowed by the law. (By 'kept private' we mean that the names of people who take part in this study will not be given to anyone else. And it means that we will only reveal what you say in a way that no one could ever guess or know it was you who said it.) If, in the course of the interview you disclose that you are, or are intending to, harm yourself or others, we are ethically and legally required to notify the appropriate authorities.

- There are three researchers involved in this project, and these three people are the only people that will know what you say. Nobody from your program will know what you say.

- All files concerning this research will be stored on the NPU secure network in a password protected file. A password is required for the opening of every file, folder, and document. Any written notes, memos, or printed documents will be stored in a locked file cabinet in the lead faculty support's office.

- The writing of the final report will be written in a way so nobody will be able to identify you. For example, all names will be changed.

\section{Any Questions?}

If you want to talk to anybody about this project; the interview, the questions, or your reactions you can talk to the interviewer or feel free to contact the principle investigator and lead faculty advisor at Department of Sociology, P.O. Box 782, Portland, OR 972000782, tel. (503) 724-6504. If you want to talk to someone else about your rights as a research participant you can contact the Chair of the Human Subject Commission of North Pacific University. Hours are 9 A.M. to 5 P.M. The offices are located at North Pacific University, Market Circle., $4^{\text {th }}$ floor, 817 SW $9^{\text {th }}$ Avenue, Portland, OR 97200. The number is 503-724-4298/1-877-460-4600, or e-mail at: hsrcc@ lists.npu.edu.

\section{If I Sign What Does It Mean?}

If you sign the consent form this means:

- You have fully read and understand this form.

- You are deciding to be a part of this research by participating in an interview.

- You are aware that you can choose not to be involved in this research. If you do agree, you can stop at anytime. It will be no problem if you change your mind and nobody will be mad.

- If you decide not to participate nobody at school will treat you differently. The program's staff will not know if you say 'yes' or 'no' to taking part in the research, and everyone will treat you the same.

- You will receive a copy of this consent form for yourself. 
Participant Signature Date

Interviewer Signature

Date
Participant name, printed

Interviewer name, printed 


\title{
Appendix C: Voluntary and Informed Consent for Mentors
}

\author{
BE PART OF AN INNOVATIVE AND IMPORTANT \\ NEW HORIZONS STUDY
}

\section{Consent Form}

New Horizons is working with Prof. E. Ramirez, from North Pacific University, to study the mentoring relationships between students and their mentors. They want to learn from you, how you built a relationship with a student protégé who is from a different background, and what that experience was like.

\section{What Will I Have To Do?}

If you are willing to participate in this interview, we will ask you to talk with us for approximately 60-90 minutes. Some of the topics for discussion will be:

- Your experience with your New Horizons student protégé.

- Your thoughts about this relationship, and its impact on your life.

- The things you have done with your New Horizons student.

\section{Are There Any Risks?}

Your relationship with your New Horizons student protégé may be an important and personal part of your life. We respect and understand this. You are not obligated to do this interview, and nobody will be upset if you do not want to. If you do decide to do this interview, you could feel uncomfortable, sad, confused, guilty, angry, or embarrassed from some of the interview questions asked. If you feel this way, you do not have to answer any question you do not feel like answering. If at any point in the interview you feel like not continuing, that is okay, and nobody will be upset, and this will not affect your relationship with New Horizons, with your protégé, or with anyone at North Pacific University. Also, if after doing the interview you feel like something upset you, you can call Dr. Eduardo Ramirez, who is the main person in charge of this study, at North Pacific University, 503-724-6504, or eramirez@npu.edu.

\section{What Will I Get In Return?}

- A chance to share your story and to help make stronger and better relationships between New Horizons mentees and their faculty mentors.

- An opportunity to help others. Lots of people get pleasure out of helping others. Your story is important and we can learn a lot from you, and teach others how to improve their mentoring relationship. This study also has the potential to contribute to providing adult mentors to minority youth who need them.

\section{What Are You Doing To Protect Me?}

Protecting your identity is extremely important to us. We want to learn from your experience, but your name is kept confidential. This is what we are doing to guarantee your confidentiality: 
- We will not tell a single person whether you decide to participate or not.

- You will be interviewed alone and what you say will be kept private.

- Nobody at New Horizons, or at North Pacific University, not even your mentee, will ever know if you did the interview, or what you specifically said.

- The interviews will be digitally recorded, and we will type it out after the interview in order to be able to read your story. But your name will be removed from the recording and the typed transcript of your interview, and we will give these files a number. The files will be stored in a password-protected computer on the North Pacific University secure network, and be password protected. We will delete them after the study is completed (no later than 4 years from the interview). Any written notes, memos, or printed documents will be stored in a locked file cabinet at the lead faculty support's office.

- Your name will never be given to anyone else. We will only reveal what you say in a way that no one could ever guess or know it was you who said it. However, if, in the course of the interview you disclose that you are, or you intend to, harm yourself or others, we are ethically and legally required to notify the appropriate authorities.

- The writing of the final report will be written in a way so nobody will be able to identify you. For example, all names will be changed.

\section{Any Questions?}

If you want to talk to anybody about this project; the interview, the questions, or your reactions you can talk to the interviewer or feel free to contact the director of the study, Dr. Eduardo Ramirez, at Department of Sociology, P.O. Box 782, Portland, OR 972000782, tel. (503) 724-6504, or eramirez@ npu.edu. If you want to talk to someone else about your rights as a participant in this study, please contact the Chair of the Human Subject Commission of North Pacific University. Hours are 9 A.M. to 5 P.M. The offices are located at North Pacific University, Market Circle, $4^{\text {th }}$ floor, 817 SW $9^{\text {th }}$ Avenue, Portland, OR 97200. The number is 503-724-4298/1-877-460-4600, or e-mail at: hsrcc@lists.npu.edu.

\section{If I Sign What Does It Mean?}

If you sign the consent form this means:

- You have fully read and understand this form.

- You are deciding to be a part of this study by participating in an interview.

- You are aware that you can choose not to be involved in this study and that if you do agree to do the interview, you can stop it at any time, and that will not be a problem, and nobody will be mad.

- The staff from the New Horizons Program will not know if you say 'yes' or 'no' to taking part in the research, and everyone will treat you the same whether you participate or not.

- You will receive a copy of this consent form for yourself.

Participant Signature Date

Participant name, printed 


\title{
Appendix D: Interview Guide for Protégé Participants \\ Questionnaire for MENTEE Interview \\ NEW HORIZONS MENTORING PROGRAM (NPU)
}

\begin{abstract}
Note to Interviewers
Demonstrate genuine and attentive listening. Keep the emphasis on their story. This means you actively work to avoid interacting with some agenda you have formed in your mind.
\end{abstract}

Attentive and useful listening is requires concentration and empathy, but you also build it into your behavior:

1. Confirm what you have heard:

"I hear you saying that... Did I get this right?... Okay, great, thanks."

2. When you get to a question which your interviewee has already talked about in part, acknowledge that fact, and build on what you already heard:

"Now I want to ask you about something that you already touched on earlier. My question is.... And I remember you saying that... Can you talk more about this...

3. Most of the questions have follow up "probing" questions. Stay away from beginning your questions or your probes with "why." 


\section{INTERVIEW}

Step 1: Introduction: What is this Interview About?

All of the people we are interviewing received a letter describing what the interview is about. And, in most of the follow-up calls, the caller also reiterated what the interview is about.

However, it is not unusual for the interviewee to show up at your interview still feeling unsure about, or not quite remembering, what you want to talk to them. This is normal. Be ready to answer that question, if and when it comes up. And if it does not come up, hit on this in the introduction:

This interview is about your experience as a mentee in the New Horizons program. You will see that in some of the interview I am going to ask about the experience of having a mentor who was from a different cultural background. My main purpose is to learn about your experience crossing a bridge of culture, or ethnicity, or race, in that relationship with your mentor. We want to learn from you what youlearned in that experience as a mentee with a mentor from a different background.

Does this sound like what you expected?! Okay, lets get started.

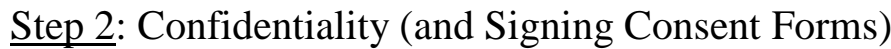

Your interview today is a real important contribution to better, stronger, mentoring for other students in the future.

This interview, I want you to know, is completely confidential. I will ask for permission to record it, because I can't talk to you, and keep up with writing notes, all at the same time. But your name, and the name of your mentor, are not going to appear anywhere when we write this out. In other words, you remain confidential.

Nobody will ever know which New Horizons mentees were interviewed, or who said what. This is what we call a confidentiality agreement. We do this so you can feel comfortable being as honest as possible in sharing your true experiences. There are not right or wrong answers to my questions. There are only your answers.

I have the confidentiality agreement here, in writing, which is a good thing, because I would not be able to share it from memory. Let me read it quickly.

Here is a copy for you.

And I need you to sign my copy, so I can show that I read it to you and you agreed. 


\section{Step 3:The Interview}

\section{Q1. Basic Facts}

(A) Were you in the New Horizons Mentoring Program in the summer of 2010, or 2011?

(B) What NCC campus were you in when you did the New Horizons program?

(C) Do you remember the name of your faculty mentor that summer?

(D) How do you identify yourself, ethnically or racially?

-Is your New Horizons mentor also <ETHNIC/RACIAL> group?

\section{Q2. Icebreaker Question:}

To break the ice, I have a bit of a funny question: Can you tell me about the funniest or strangestmoment you ever had with your mentor?

That was funny...

\section{Q3. Your New Horizons Mentor:}

You said your mentor was < Mentor name>. Can you please describe < Mentor name to me> as a person?

Q4. How did you first meet your mentor? Was starting a relationship with this mentor your choice completely, or did you get matched with her/him?

\section{Q5.[Interviewer: FYI, the theme here is the relationship timeline]}

You told me already that did the New Horizons program in the summer of
(A) Do you remember how you first met your mentor?
(B) That summer, how often would you and <mentor> usually meet?
(C) How much time did you get to spend with your mentor?
(D) Did you continue meeting your mentor after that summer?

Q6. [Interviewer, FYI, the theme here is: First Impressions of your Mentor] 
Think back to when you first met <MENTOR NAME>. Imagine you had never shared time with your mentor again after that first meeting. What impression did your mentor make?

Probes:

-- (At that first meeting) Did you feel self-conscious, and I mean by this, did you feel

"what is this person thinking about me"?

- How do I look to this person?

- What am I doing here with this person?!

$-\ldots .$.

-Did you make a connection immediately?

- Did you feel your mentor and you were very similar, or did it feel like you were meeting someone who was very different?

- What differences stood out? (Culture? Language? Race? Gender? Class??)

(Take note of the differences noted, and probe:)

- Had you ever had a <<race/culture of mentor >> mentor before?

- Do you remember feeling that it would be nicer if your mentor were $<<$ mentee race/ethnicity > like you?

\section{Q7. Things You Did With Your Mentor}

What did you work on with your mentor?

- Tell me, what kinds of things would you and mentor do when you met or shared time? - What did you enjoy the most? What did you get the most out of?

\section{Q8. [Interviewer, FYI, the theme here is: how "real" was the relationship]}

I would like to talk a little about moments when you maybe felt a connection with your mentor, if you ever felt this way.

Did you ever feel like you made a real connection? (We all have a sense of a real connection is, as opposed to one that does not feel real. So whatever "a real connection" means to you is fine)

If Yes:

- Okay, can you talk about what made the connection real to you?

If No:

- Okay it seems like the connection with your mentor did not feel close a lot of the time. Can you talk about this? What do you feel was missing. 
Q9. [Interviewer, FYI, the theme here is: More on the Mentee's feelings as the program progressed]

A few minutes ago you talked about some of the main things you worked on together with your mentor... (Interviewer, here we build on what mentee has talked about in Q7.)

- When you met, who initiated meetings

- When you worked on <<Item 1 from Q7>>:

- Who's idea was it to work on that?

-(If mentor's): how much input did you have?

- What concrete things did you accomplish?

- Was your mentor important in getting you there?

- Was your mentor important in getting you to discover something about yourself?

Interviewer, if there were more items in Q7, repeat same questions for up to three items.

[Interviewer, FYI, the theme of the next two questions is: Mentee's perception of how the structure of the New Horizons Porgram influenced the relationship between self and mentor]

Q10. Is there something about the New Horizons program that made it easier for you to connect and build a good relationship with <MENTOR'S NAME〉

- I hear you saying that <<PROGRAM $>>$ does feel this is part of why you connected with $\langle<$ MENTOR $>>$ , and you

-- Are there other things you feel $<<$ PROGRAM $>>$ should do?

Q11. Is there something about the New Horizons program that made it easier for you, SPECIFICALLY, to build a relationship with a mentor who is from a different culture/ethnicity/race than you?

-I hear you saying that <<PROGRAM $>>$ does which helped you make a real connecting with a mentor from a different race ethnicity.

-- Are there other things you feel <<PROGRAM >> could do to help connect with mentors of a different culture/race/ethnicity?

Q12. [Interviewer, FYI, theme here is: Changes in First Impressions] 
I asked you at the start of the interview to imagine you never shared time with your mentor again after the first time you two met, and to tell me, what impression of $<$ Mentor's name> you would have walked away with?

-You said

- Do you still see you mentor the same way?

- How has this changed from the first time you met $<<$ mentor $>>$ ?

- Do you still feel self-conscious when you meet with your mentor? Do you feel "what is this person thinking about me?

- After knowing getting to know your mentor for <<TIME $>>$, have you discovered you and your mentor have things in common?'

- Were you surprised by any of these things in common?

- Was it important to discover these things in common?

\section{Q13. After your experience with New Horizons:}

- Do you feel any differently now about working with a mentor who is from a different race or ethnicity?

- Do you feel this experience will make it easier for you in the future to connect a person of a different race or ethnicity who could be a useful mentor at school, or in a career?

\section{Q14. What if...}

Tell me, what if your mentor in the New Horizons Program had been $<$ Black/Latino/American Indian/Asian/White> like you?

- Would things have been different?

- Would you have gained something different?

- Would you have missed out on something?

\section{Q15. Do you think you would like to be a mentor in the future?}

- How would you feel mentoring someone of a different racial or ethnic background from yourself?

\section{CLOSING}

Well, that is the interview. I have really enjoyed talking to you about your experience with the New Horizons. I know that your experience, and the experience of all other New Horizons mentees being interviewed, is going to teach us a lot about mentoring that to 
connects mentees and mentors from different ethnic, cultural, or racial background. Thank you very much for your time. 


\section{Appendix E: Interview Guide for Mentor Participants}

\section{INTERVIEW \\ Step 1: Introduction: What is this Interview About?}

All of the people we are interviewing received a letter describing what the interview is about. And, in most of the follow-up calls, the caller also reiterated what the interview is about.

However, it is not unusual for the interviewee to show up at your interview still feeling unsure about, or not quite remembering, what you want to talk to them. This is normal. Be ready to answer that question, if and when it comes up. And if it does not come up, hit on this in the introduction:

This interview is about your experience as a mentor in the New Horizons Mentoring program. You will see that in some of the interview I am going to ask about the experience of having a student protégé who was from a different cultural background. My main purpose is to learn about your experience crossing a bridge of culture, or ethnicity, or race, in that relationship. We want to learn from you what youlearned in that experience as a mentor with a student protégé from a different background.

Does this sound like what you expected?! Okay, lets get started.

\section{Step 2: Confidentiality (and Signing Consent Forms)}

Your interview today is a real important contribution to better, stronger, mentoring relationships in the future.

This interview, I want you to know, is completely confidential. I will ask for permission to record it, because I can't talk to you, and keep up with writing notes, all at the same time. But your name, and the name of your student protégé, is not going to appear anywhere when we write this out. In other words, you will remain confidential.

Nobody will ever know which New Horizons mentors were interviewed, or who said what. This confidentiality agreement is so you can feel comfortable being as honest as possible in sharing your true experiences. There are not right or wrong answers to my questions. There are only your answers.

I have the confidentiality agreement here, in writing, which is a good thing, because I would not be able to share it from memory. Let me read it quickly.

Here is a copy for you. And I need you to sign my copy, so I can show that I read it to you and you agreed. 


\section{Step 3:The Interview}

\section{Q1.Basic Facts}

(A) What department are you affiliated with here at North Pacific University?

(B) Were you a mentor in the New Horizons Mentoring Program in the summer of 2010, or 2011 ?

(C) Do you remember the name of the student that worked with you that summer?

(D) How do you identify yourself, ethnically or racially?

-Is your Bridges student protégé also <ETHNIC/RACIAL> group?

If they answer "No"

- Do you know what race or ethnicity <NAME OF PROTÉGÉ> identifies with?

\section{Q2. Icebreaker Question:}

Let me break the ice with an easy question! To break the ice, I have a bit of a funny question: Can you tell me about the funniest or strangestmoment you ever had with <PROTÉGÉ NAME>?

That was funny...

\section{Q3. Your New Horizons Protege:}

You said your student protégé was < Protégé name>. Can you please describe <Protégé name> to me as a person?

\section{Q4. How did you first meet <PROTÉGÉ NAME>?}

Was starting a relationship with this student your choice completely, or did you get matched with her/him?

Q5. Do you remember the first meeting you had with <PROTÉGÉ NAME> Can you tell me about that first meeting?

Q6. Imagine you had never shared time with <PROTÉGÉ NAME> again after that first meeting. What impression did he/she make?

Connection immediately?

Either of you feel awkward?

Pick up on obvious similarities between the two of you?

Any concerns about connection? 
Q7. Tell me a bit about the PROJECT worked on and what did that summer?

- Summer routine?

- How often TOGETHER

- How much INDEPENDENT

Q8. Did you and do any things outside of the usual research routine?

Q9. Did feel that a real bond or connection developed that summer?

If Yes:

- Special moments / incidents

- Enjoyed the most?

If No:

- Why do you think this was?

Q10. After the summer was over, did you ever see again? Do you still see ?

- Protégé come back to you for anything?

- Social?

Q11. Can you tell me how you came to the decision to be a mentor with the Bridges Program?

- Have you mentored before?

- Ever have a minority protégée before?

Q12. Turning to expectations and goals.... Did the experience mentoring meet the expectations and goals you may have had?

- As a mentor, what did you expect this role to involve?

- Ways helped prepare for the transition from community college to a fouryear university? 
Q13. Did New Horizons, the program, do anything in particular that made it easier for you and to connect and build a relationship?

-- Are there things you feel New Horizonsshould do/ could do to support the mentorprotégé pairs?

Q14. Did New Horizons do anything that help you build a relationship with a student who is from a different culture/ethnicity/race than you?

-I hear you saying that New Horizons does , which

helped you make a real connecting with a student from a different race.

-- Are there other things you feel New Horizons could do to help mentors connect with students of a different culture/race/ethnicity?

Q15. Did the experience as a New Horizons faculty mentor... impact how you think about mentoring ethnic or racial minority students in the future?

Q16. Imagine had not been from a minority background... how might it have been different?

- ease/comfort?

- more/less/equally/differently meaningful?

Q17. Did this experience influence how you feel about being a mentor again in the future?

We have reached the end of my interview, and I would like to share back with you the overall picture that I have got. Please tell me if you feel I got it right or not...

Well, this is the end of the interview. I really can't thank you enough for taking time away from your busy schedule to share your story. I've really enjoyed talking to you about your experience mentoring and I know that your experience, and the experience of all the 
other New Horizons mentors being interviewed, is going to teach us a lot about mentoring that connects mentees and mentors from different ethnic, cultural, or racial background. Thank you again for your time. 